\title{
Broken Connections: The Evidence for Neuroglial Failure in ME/CFS (1)
}

\author{
Herbert Renz-Polster, $M D^{(2)}$; Dorothee Bienzle, $P h D^{(3)}$ \\ Note: the hypothesis layed out in this preprint has been further developed and is now published in \\ Frontiers Cellular Neuroscience: https://www.frontiersin.org/articles/10.3389/fncel.2022.888232/full
}

"On the one hand, it is clear the underlying pathology involves the nervous system, energy metabolism, and the immune system. On the other hand, it is not clear what ties together the pathology seen in these different systems, nor which of the abnormalities came first, nor what triggered that first abnormality". (Anthony L. Komaroff) ${ }^{1}$

\section{Abstract}

In spite of decades of research, the pathobiology of Myalgic Encephalomyelitis/Chronic Fatigue Syndrome (ME/CFS) is still poorly understood. Several pathomechanisms have been identified including immune abnormalities, inflammatory activation, mitochondrial dysfunction, endothelial dysfunction, muscular dysfunction, cardiovascular dysfunction, and dysfunction of the autonomous nervous system. Yet, it remains unclear how these pathways are related, which of them may be upstream or downstream, and which ones may be explanatory of the symptomatology of ME/CFS (and thus possibly targets for therapeutic interventions). A similar uncertainty is currently experienced by thousands of researchers who struggle to understand Postacute Sequelae of Covid (PASC, "Long Covid"), a condition with many similarities to ME/CFS.

In this paper, we present a theoretical strategy that may help clarify the causal chain of pathophysiological events in ME/CFS. We propose to focus on the common final histological pathway of ME/CFS. I.e., we suggest to ask: Which cellular compartment may explain the pathological processes and clinical manifestations observed in ME/CFS? Any functional unit consistently identified through this search may then be a plausible candidate for further exploration.

\footnotetext{
${ }^{1}$ Current version to be downloaded at: https://osf.io/ef3n4

${ }^{2}$ Mannheim Institute of Public Health, Social and Preventive Medicine, University Medicine Mannheim, Heidelberg University, 68167 Mannheim, Germany. Corresponding author: Herbert Renz-Polster, e-mail: Herbert.Renz-Polster@kinderverstehen.de

${ }^{3}$ Professor of Veterinary Pathology, University of Guelph, Canada
} 
For this "histological" approach we have compiled a list of 22 undisputed clinical and pathophysiological features of ME/CFS that need to be plausibly and most directly explained by the dysfunctional cellular unit in question. For each feature we have searched the literature for pathophysiological explanations and analyzed if they may point to the same functional cellular unit.

Through this search we have identified the CNS neuroglia - microglia and astroglia - as the one functional unit in the human body which may best explain all and any of the clinical and pathological features, dysfunctions and observations described for ME/CFS. While this points to neuroinflammation as the central hub in ME/CFS, it also points to a novel understanding of the neuroimmune basis of ME/CFS. After all, the neuroglial cells are now understood as the functional matrix of the human brain connectome which operates beyond and above specific brain centers, receptor units or neurotransmitter systems and integrates innate immune functions with CNS regulatory functions pertaining to autonomous regulation, cellular metabolism and the stress response.

Of note, this is not a unifying theory about the etiology, the triggers or the inception process of ME/CFS. This approach is focused solely on finding the final pathogenetic pathway(s) which may underlie the clinical manifestations of ME/CFS. It does not question existing theories about the inception of ME/CFS, be they based on autoimmunity, persistent infection, mitochondrial or metabolic failure or any other assumption. 


\section{Table of Content}

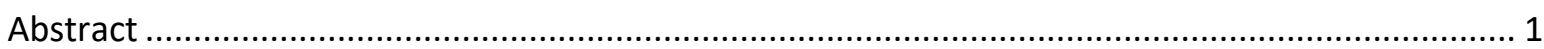

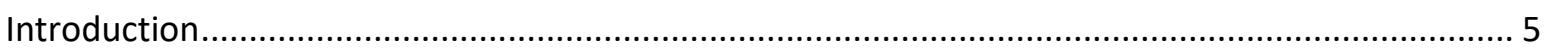

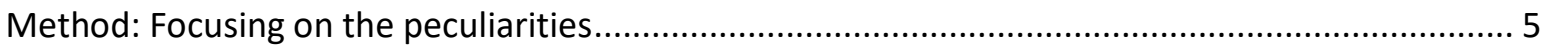

Our assumptions for this "Occam razor" investigation ................................................................ 7

1.0 Evidence \#1 The neuroglial unit orchestrates the many physiological functions that have so far been implicated in ME/CFS ............................................................ 11

2.0 Evidence \#2: The clinical features of ME/CFS can all be explained as a consequence

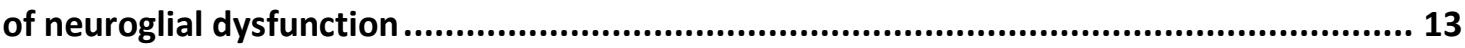

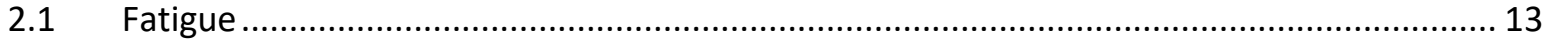

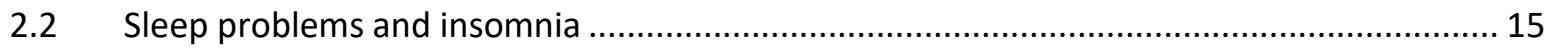

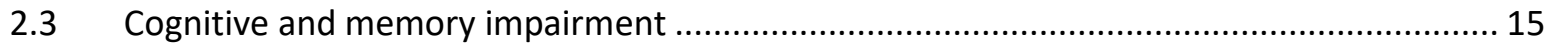

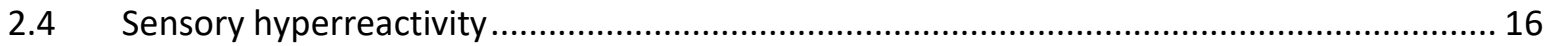

2.5 Psychomotor slowing and the "coupled" nature of ME/CFS ............................................. 16

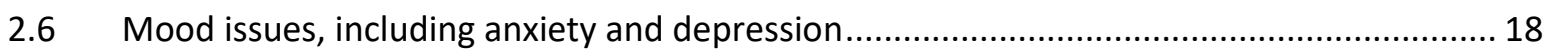

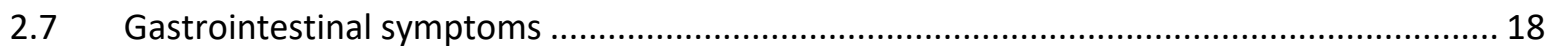

3.0 Evidence \# 3 The core feature of ME/CFS, post exertional malaise, can be explained as a consequence of neuroglial dysfunction .................................................................... 19

4.0 Evidence \#4 The pathological features of ME/CFS can be explained as a consequence

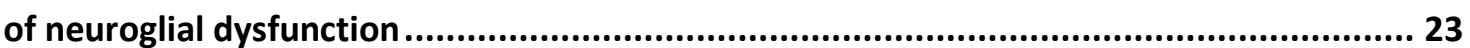

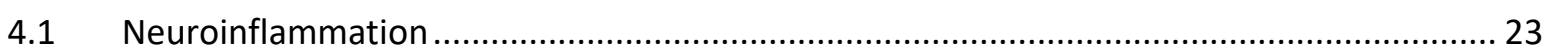

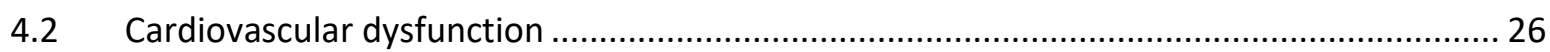

4.4 The inflammatory - and possibly autoimmune - nature of ME/CFS................................... 29

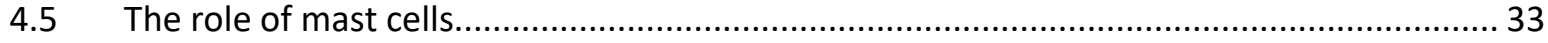

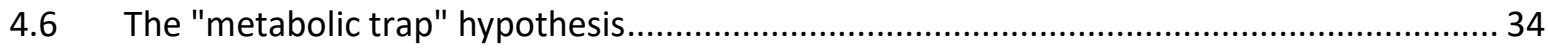

4.7 Mitochondrial, cellular and "metabolic" dysfunction .......................................................... 36

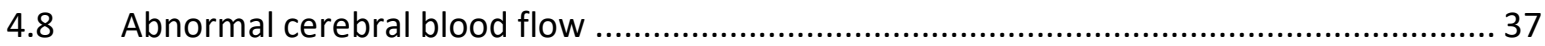

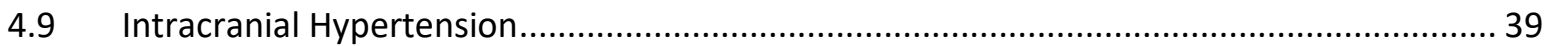

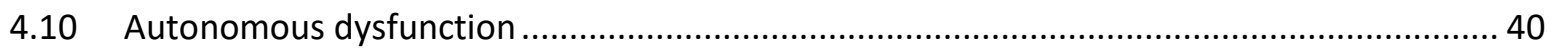

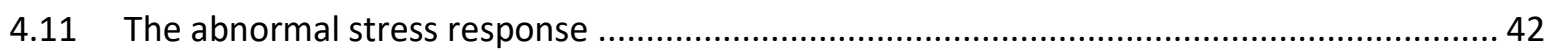

5.0 Evidence \#5 The "hypermobility" and "neuro-orthopedic" enigma .................................. 45

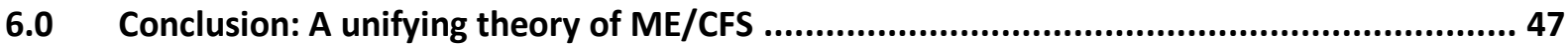

Question 1: If it is all in the CNS: may there be a part of the CNS than can be considered the tectonic hotbed of the ME/CFS pathology? 
Question 2: With so many pathogenetic links clearly identified in ME/CFS research (including hypoperfusion, inflammation, abnormal adrenergic signaling etc.) - with which link could the pathogenetic chain in ME/CFS start?

7.0 The big question: Which event(s) may trigger the whole process? What may be the initiating event of ME/CFS?

8.0 COVID-19 to the rescue

References

A personal note from the main author

I am not a neuroscientist (in fact I am a pediatrician trained in pediatric pulmonology). From my other research interests however, and as a longtime editor of a medical textbook, I am versed in reading and understanding the scientific literature. I have thoroughly reviewed the available research record on ME/CFS; I am also familiar with the clinical aspects of this disease because I have been suffering myself from ME/CFS for more than four years. This experience - which has taken my life (and the life of my family) hostage as it has done with untold numbers of lives - is the very motivation for this work.

This working paper covers many research areas, from basic physiology to clinical medicine. While I have tried my best, there may be pieces that I may have inadequately understood or misinterpreted. If you notice any errors or omissions, or if you want to critique or comment on this paper, please drop me a line. 


\section{Introduction}

For research, ME/CFS poses a complex challenge. Clinically, the syndrome presents with a wide array of non-specific symptoms like fatigue, non-refreshing sleep, cognitive impairment, mood changes or motor slowing, i.e., symptoms which widely overlap with other disorders and are just too common to inform about pathomechanisms possibly specific to ME/CFS. Adding to this non-specific complexity, the disease presents as a mélange of mental, cognitive and somatic impairments, some of which are clearly indicative of central nervous system dysfunction while others point to peripheral dysfunction, putting the question center stage: Where does all this begin? In the brain or in the periphery? Or with some systemic process involving both?

Indeed, the pathological level of ME/CFS is no less complex than the clinical plane. Pathologically, $\mathrm{ME} / \mathrm{CFS}$ undisputedly represents as a multiorgan disease that can optionally be described as encephalopathy, myopathy, dysautonomia, mitochondriopathy, vasculopathy or immunopathy again raising the question: How do all these "pathies" fit together? What is upstream, what is downstream? And again: Where does all this start?

Answering these questions is additionally hampered by the fact that the abnormal pathological findings lack a pathognomonic signature. In most, if not all, patients there is evidence for associated immune dysfunction, cerebral hypoperfusion and autonomic dysregulation; in an unknown proportion of patients there is also evidence for mitochondrial/cellular dysfunction, autoimmunity, small fiber neuropathy, endothelial dysfunction, beta-2-dysfunction, neuroinflammation and cardiovascular pathology. But as striking as the combination of dysfunctions may appear, these findings are not specific of ME/CFS but also typical of many other diseases with or without clinical overlap with ME/CFS. So how can any of these pathological phenomena explain ME/CFS?

With all these descriptions of diffuse, non-specific characteristics of ME/CFS we haven't even touched on the central problem of this disease - a problem that may be most relevant for any search of an explanatory framework for the pathogenesis of ME/CFS: We do not even know if ME/CFS represents a pathologically distinct category. There is clear evidence that ME/CFS is made up of different etiological entities (which we call "etiotypes"), with at least three categories distinguishable: a rare( $r$ ) form with an "orthopedic-neurological" background (ME/CFS related to craniocervical instability, craniospinal stenosis, spinal/cranial trauma or tethered cord - diagnoses sometimes related to Ehlers-Danlos or other hypermobility syndromes), a common form triggered by overwhelmingly stressful immunological events (like microbial infections, psychological trauma, combat experience with possible chemical exposures or operations/anaesthesia) and another common form with protracted, insidious onset without known triggers. Unfortunately, as of yet, it is not known if the clinical "ME/CFS" manifestations associated with these "etiotypes" also share a common pathomechanism.

So, while several links of the pathological chain in "ME/CFS" are clearly on the table there is no description of how these links are interlocked - and no information if they may be interlocked in the same order in all etiotypes of ME/CFS.

\section{Method: Focusing on the peculiarities}

But ME/CFS is not only an enigma, not only complex and not only non-specific. ME/CFS also presents with clinical features and symptom constellations that are unique in the kingdom of diseases (and this, by virtue of the clinical definition of ME/CFS, applies to all etiotypes of ME/CFS, like post 
exertional malaise, for instance). Moreover, some features, while encountered in many other diseases, are "packaged" in peculiar ways providing characteristic patterns, imprints or even hallmarks of ME/CFS. Clearly, there are specific leads in the ME/CFS landscape that beg to be followed.

Theoretically, one could follow these leads in different directions. Classically, the question has been: which pathological mechanism may best explain the phenomenon in question? This has resulted in a plethora of explanations of ME/CFS like the Wirth-Scheibenbogen hypothesis (in which endothelial dysregulation of both the peripheral and the central vessels takes center stage $)^{2}$, the vanElzakker hypothesis (focusing on vagal infection/dysfunction), ${ }^{3}$ the neuroinflammatory hypotheses by Mueller and Younger, ${ }^{4}$ Barnden ${ }^{5}$ and others (focusing on brainstem neuroinflammation), the cerebral hypoperfusion hypothesis by Visser, ${ }^{6}$ the dysfunctional hypothalamus hypothesis by Mackay, ${ }^{7}$ the "sustained arousal"/dysfunctional nucleus coeruleus hypothesis by Wyller, ${ }^{8}$ the capillary stress hypothesis by Ron Davis, ${ }^{9}$ the herpesviral reactivation hypothesis by Prusty ${ }^{10}$ or the "cell danger response" by Naviaux, ${ }^{11}$ to name just a few.

However, these searches for the "primary" pathological process in ME/CFS all share a common problem. They may all be "correct" - yet, the question remains if they can be explanatory in a causal sense. After all, the pathological phenomena described may either co-exist or they may interact. Or they may be consequences of higher order phenomena.

There are other theoretical ways to follow the leads in question, like proteomic, metabolomic, gene expression ("transcriptomic"), or epigenomic pattern recognition studies. A biochemical-genetic modelling study, for example, has tried to identify distinct biochemical pathways that may be disrupted in ME/CFS - and has come up with a specific "metabolic trap" hypothesis revolving around the kynurenine/tryptophan metabolic pathway (an approach that we will revisit, see [4.6]). Hypotheses have also been generated on the basis of functional-topographical considerations, for example the hypothesis of a dysfunctional locus ceruleus, ${ }^{12}$ a dysfunctional paraventricular nucleus, ${ }^{7}$ a dysfunctional suprachiasmatic nucleus, ${ }^{13}$ or basal ganglia defects. ${ }^{14}$ Others have tried to pinpoint $\mathrm{ME} / \mathrm{CFS}$ pathology in the various transmitter systems of the CNS, and suggested abnormalities in the dopaminergic, ${ }^{15}$ serotoninergic, ${ }^{16}$ adrenergic, ${ }^{2}$ GABA-ergic ${ }^{17}$ or glutaminergic ${ }^{18}$ transmitter systems.

Similarly, and this is what we are proposing, one could follow the available leads - be they clinical or pathological - to their "histological" or cellular functional source(s). This approach would take all the characteristic features of ME/CFS one by one and ask: which cellular compartment or functional unit may most plausibly explain this phenomenon? Of course, this approach may identify multiple different sources. But what if the analysis of the many pathogenetic links in ME/CFS pointed to the same functional unit?

One could call this approach a search for cellular-functional plausibility: Is there a functional unit in human biology that may be capable of orchestrating ALL phenomena noteworthy about ME/CFS?

It is our informed conviction that by following the leads of "all what is known about ME/CFS" we may come up with a "functional hot spot" that may indeed be the pathogenetic hub in ME/CFS where all the pathomechanisms intersect. This hot spot may then be the sweet spot for further research. 


\section{Our assumptions for this search for the pathogenetic hub of ME/CFS}

So, in a sense this working paper represents an extended "Occam razor" investigation: what is the single most parsimonious explanation that is able to clarify all of the complex features of ME/CFS? Any causal explanation of ME/CFS must be able to satisfactorily explain the following characteristic features of ME/CFS:

Feature 1: ME/CFS has a quasi-pathognomonic feature: post-exertional malaise. While ME/CFS patients all have very different baseline levels of functionality, they all have one peculiar feature in common: a uniform reaction to stressful events, variably termed post-exertional malaise, PEM, or Post Exertional Neuroimmune Exhaustion, PENE. In its strict definition (exercise-induced, delayed exacerbation of symptoms lasting over 12 hours) PEM may even be unique to ME/CFS - even more so, if one considers the characteristics of ME/CFS associated PEM:

- The clinical deterioration appears with a specific delay of at least several hours to one day post exercise and typically lasts several days (from 12 hours to several weeks) .

- The exacerbation does not follow a dose-response dynamic but an individually calibrated threshold dynamic: Each ME/CFS patients seems to have an individual, severity dependent threshold for setting off this post-exertional reaction, ranging from very light to vigorous exercise.

- The exacerbation has multi-level triggers. While PEM can be triggered by muscular exercise ALONE, it can, in the same patient, also be triggered by cognitive or mental exercise ALONE, i.e., in the complete absence of muscular exertion.

This exercise-induced, threshold-driven, delayed form of disease exacerbation is so central to $\mathrm{ME} / \mathrm{CFS}$ that any theory of ME/CFS must provide a clear explanation of these characteristics of PEM.

Feature 2: In ME/CFS, central and peripheral manifestations are always "coupled". Another noteworthy feature about ME/CFS is a typical, specific, uniform and pervasive "packaging" of symptoms. Mental fatigue and muscular fatigue are simultaneously present, always. The more a patient suffers from cognitive and mood disturbance, the less his or her muscles work. The more centrally fatigued a patient, the slower he or she walks. Central sensory dysfunction like hypersensitivity to noise, light or touch goes hand in hand with decreased exercise capacity. Cognitive dysfunction goes hand in hand with poor skin perfusion. Also, as disease severity increases, both mental and motor dysfunctions deteriorate in union, i.e., the less functional a patient is the more pronounced his central AND peripheral disturbances. Conversely, amelioration, temporal improvement or recovery from PEM are similar tandem processes. In short, the organ levels on which ME/CFS presents seem to be coupled. Any theory about ME/CFS must be able to explain this central-peripheral clinical coupling (as well, ideally, as give a clue as to its underlying direction: do central versus peripheral phenomena lead or follow?)

Feature 3: ME/CFS - or a ME/CFS-like disorder - can be caused by specific neuro-orthopedic conditions. This feature may be the most tenuous one. Yet, for research purposes, it may be unusually fertile because it is so unusual and so clearly tied to a plausible pathomechanism. Indeed, from a theoretical-logical standpoint, this feature could turn out to be a crucial entry point to the deepest possible understanding of ME/CFS. 
The protagonist of the movie "Unrest" has given the world an inside glimpse of what ME/CFS looks like - being bedbound, hypersensitive to noise and light, having severe crashes after exercise... - the whole ME/CFS package. This person now runs half-marathons, she is completely cured of her "ME/CFS". ${ }^{19}$ Her condition turned out to be caused by cervico-cranial instability $(\mathrm{CCl})$ combined with a tethered cord syndrome. Her "ME/CFS"-symptoms all vanished after surgical correction. Numerous other case reports have confirmed that several neuro-orthopedic conditions like Tethered cord syndrome, Chiari malformation, cervical stenosis, venous stenosis, median arcuate ligament syndrome (MALS), Eagles syndrome and cranio cervical instability can perfectly mimic - or cause? - ME/CFS.

Clinically, these histories are all accounts of ME/CFS - the diagnoses persist even when the strictest diagnostic criteria for ME/CFS are being applied. They are all accounts that may also explain a related, highly conspicuous peculiarity: The fact that cranio-cervical instability and the hypermobile form of Ehlers Danlos Syndrome (hEDS) - a genetic condition that sets people up for joint hypermobility, but also intracranial and immune related abnormalities - is widely overreported in ME/CFS sufferers (for details, see [5.0]).

These associations may indeed point to the fact that a significant portion of ME/CFS patients may have underlying neuro-orthopedic and/or connective tissue disorders underlying their ME/CFS. Any theory about ME/CFS to be taken seriously must either prove that the neuro-orthopedic and "hypermobile" cases of "ME/CFS" are indeed NOT ME/CFS cases (because they may have distinct features that set them apart, for instance) or explain why these conditions are able to mimic - or trigger - the clinical picture that is called ME/CFS by scientific consensus.

Feature 4: ME/CFS has other compelling and possibly specific clinical features. While the fatigue so central to ME/CFS is shared with a vast number of other, surprisingly dissimilar diseases or conditions like Hepatitis C infection, Multiple Sclerosis (MS) or chemotherapy, there is a set of concomitant clinical features that are not present in most other disorders in the fatigue category. These features are indeed so unusual and typical of ME/CFS that they may provide better access to the understanding of ME/CFS and clearly beg for an explanation of the underlying pathological phenomena:

- the hypersensitivity to sensory stimuli across sensory modalities (hypersensitivity to light, noise, touch, odors and sensory overload in general) is not only typical of ME/CFS but also seems to correlate well with clinical severity as it is most likely to be found in the severely affected patient group and during PEM exacerbations. ${ }^{12}$

- $\mathrm{ME} / \mathrm{CFS}$ patients display an "enigmatic" form of cardiovascular compromise marked by low blood volume, low stroke volume, chronotropic incompetence (i.e. inability to reach normal levels of heart rate, stroke volume, and oxygen loading during exercise), paradoxically low Renin-Angiotensin-Alsosterone-System (RAAS) activity, intermittent fluid retention, compromised microcirculation and clinical signs and symptoms of orthostatic intolerance in some patients. ${ }^{2,20}$

- $\mathrm{ME} / \mathrm{CFS}$ presents with another paradox. Unlike in other fatiguing diseases ME/CFS is marked by a combination of fatigue and excessive arousal. Indeed, the fatigue so central to the disease and so severe at times, is clinically coupled with an opposing picture of "sustained arousal" 8 marked by hypervigilance, signs of adrenergic hyperstimulation, insomnia and often profound emotional distress (including panic and anxiety). On the pathological plane a similar paradox can be observed (if related or unrelated): adrenergic overstimulation and adrenergic failure co-exist: the sympathetic autonomous system is in overdrive, yet the "fight or flight response" fails to increase muscular blood flow or availability of energy. How 
can this coexistence of "hibernation" and "sustained arousal" be explained? The autonomous paradox may even ectend to a more profound level: while the sympathetic nervous system is in overdrive, there is, at the same time, evidence of vagal dysfunction. The whole autonomous regulation seems to be in failure.

- This, however, may not be the last paradox to be explained about ME/CFS. ME/CFS is described as the most vicious disease in the hell of diseases causing the most severe functional restrictions with patients being completely incapacitated and suffering lower quality of life than patients with MS or many late stage cancers. ${ }^{21}$ Also, in ME/CFS, there is clear evidence of a multi-system involvement including chronic inflammatory and possibly autoimmune stimulation. And yet, ME/CFS - secondary damage from complications notwithstanding, which of course are also observed - seems to leave little traces when it comes to tissue damage: ME/CFS is not a neurodegenerative disease nor generally a chronic degenerative disorder. In spite of severe derangements and chronic inflammatory processes the defects seem to remain functional in nature rather than destructive or cytopathic. This too is noteworthy and needs to be explained.

\section{Feature 5: While many pathological features have been identified in ME/CFS patients, only a few} are consistently found in ALL of them. ME/CFS has been shown to be associated with a vast array of pathological processes, including possible neuroinflammation, activation of the immune system, chemoreceptor dysfunction, baroreceptor dysfunction, increased intracranial pressure, cerebral hypoperfusion, disordered neural connectivity, vagal dysfunction, brainstem dysfunction, autoimmunity, cellular dysfunction, small fiber neuropathy, endothelial dysfunction as well as muscular and cardiovascular dysfunction (these pathological processes will all be highlighted in this paper, which is one of the reason for its substantive size). However, there is increasing recognition that some of these processes are present only in a subset of ME/CFS patients while others may be present in ALL cases of ME/CFS - the latter including evidence of an activated immune system, cerebral hypoperfusion and autonomous dysfunction. Any explanation of ME/CFS must be able to explain this core pathogenetic signature.

\section{The critical criteria in summary}

To summarize, anyone who wants to present a unifying theory about the pathogenesis of ME/CFS (and, to be precise, of the ME/CFS-like cases of PASC) must explain the following features, in full:

- he or she must be able to explain the central clinical phenomenon of ME/CFS, i.e., post exertional malaise: Why does this exercise-induced exacerbation of ME/CFS symptoms always set in with a specific time delay and duration? Why does it follow an individually calibrated threshold dynamic? Why can it be equally triggered by physical exertion or by mental/emotional exertion or by cognitive exertion?

- she or he must be able to explain the different etiotypes of ME/CFS, esp. the "hypermobile" and "neurologic-orthopedic" cases: which pathomechanism may explain that both immunological and primarily "mechanical" insults may induce a similar symptomatology?

- he or she must be able to explain the clinical and pathophysiological paradox of ME/CFS, i.e., the co-existence of under- and overarousal in the clinical picture as well as the co-existence of adrenergic stimulation and adrenergic failure on the pathological plane 
- he or she must be able to explain the core pathological features observed in ME/CFS like cerebral hypoperfusion, autonomous dysfunction, immune dysregulation/inflammatory stimulation, peripheral vascular and muscular dysfunction as well as cardiovascular dysregulation

- he or she would also have to explain the uniform and pervasive coupling of central and peripheral symptoms in ME/CFS

- and finally, and ideally: he or she should be able to explain not only all and any of the symptoms associated with ME/CFS but also explain how the few successful therapeutic interventions on record so far may be explainable as to their mode of action.

As a caveat and for clarification we want to add an important qualifier. It is unknown if ME/CFS can be legitimately conceptualized as manifestation of a single, underlying pathophysiological process. Indeed, many ME/CFS researchers question a universal pathological matrix especially across the different etiotypes of the disease. This may be seen as a discouragement or even fatal blow to the approach presented in this working paper.

We disagree for two reasons. Eeven if ME/CFS turned out to be a clinical entity with different pathological root causes the Occam razor approach presented here should still be informative as it may be used for generating hypotheses about the very nature of these possibly disparate ME/CFS "pathotypes". Also, even if the pathological matrix of ME/CFS may be polymorphous, the striking similarity, uniformity und uniqueness of some clinical features both in terms of triggers and clinical appearance may suggest at least some shared pathological pathways - which may be identified exactly through the inferential approach laid out in this paper. Even delineating common denominators between the different etiotypes would be a huge step forward in understanding "ME/CFS". 


\section{Why we should study the glial compartment - the evidence}

\subsection{Evidence \#1}

\section{The neuroglial unit orchestrates the many pathological functions that have so far been implicated in ME/CFS}

Astrocytes are the neural cells of ectodermic origin and predominant glial cells in the brain, the astrocyte compartment as a whole is therefore also termed astroglia. Microglia are the CNS-resident innate immune cells of mesodermal origin involved in tissue defense and maintenance. Although astroglia and microglia (together termed neuroglia) are heterogenous cell populations they both cooperate in many functions. The latter is especially true for regulatory influences on the innate immune system and pro-inflammatory processes. ${ }^{22-24}$

- Astroglial cells are an independent functional unit securing adequate cerebral blood flow. The regulation of brain perfusion happens via local production of vasoactive molecules like prostaglandins and nitric oxide but also through the modulation of cardiovascular reflexes. ${ }^{25}$ Indeed, it has been shown that astrocytes influence the sensitivity of the intracranial baroreflex, ${ }^{26}$ detect falling cerebral perfusion pressure and are able to activate CNS autonomic sympathetic control circuits that in turn increase systemic arterial blood pressure and heart rate in order to maintain brain perfusion and oxygenation. Besides astrocytes, microglia also plays a role in the auto-regulation of cerebral blood flow (capillary-associated microglia has been shown to regulate capillary dilation). ${ }^{27}$

- Astroglia also takes part in information transfer and processing in the CNS, in part by virtue of its differential expression of receptors for a variety of signal molecules such as dopamine, serotonin $^{28}$, estrogens ${ }^{29,30}$, glutamate ${ }^{31,32}$, gamma-aminobutyric acid (GABA), ${ }^{33}$ norepinephine ${ }^{34}$ and $\mathrm{CRF}^{35}$ The latter makes the neuroglial compartment an important player in the stress response (here again, the two players of neuroglia act together).

- The neuroglial unit also modulates circuits involved in learning and memory, ${ }^{36,37}$ control of sleep, ${ }^{38}$ and regulation of breathing ${ }^{39,40}$ and other autonomic functions (e.g. via the nucleus of the solitary tract). ${ }^{41}$ Indeed, the astroglial unit can be conceptualized as a set of switches within the CNS that can block or propagate stimuli along neural tracts. ${ }^{42}$

- Astrocytes actively secrete and absorb neurotransmitters and carry most of the extracellular glutamate. Indeed, astrocytes can accumulate up to $80 \%$ of total glutamate released in the brain. The astrocyte compartment therefore plays a unique role in limiting glutamate excitotoxicity.

- Astroglia secures and controls the blood-barrier function by wrapping their end-feet around the cerebral capillaries. ${ }^{43}$ Thus, astroglia - in cooperation with microglia - is involved in the control of the immigration of peripheral immune cells and also the major player in maintaining adequate intracranial pressure and preventing intracranial hypertension. 
- Neuroglial cells with their abundantly expressed TLR4 receptors are extremely sensitive to pro-inflammatory signals from the innate immune system. ${ }^{44-46}$ Upon TLR4 activation glial cells become activated and then start to produce proinflammatory factors such as TNF-alpha, interleukin-1 beta and IL6. In this sense astrocytes are both resonators and inducers of neuroinflammation. As a consequence of this activation astroglia and microglia become hyperresponsive to any further immunological stimulation, resulting in a self-propelling cycle of neuroinflammation. ${ }^{47}$

- The role of astroglia and microglia in neuroinflammation however is flexible. Astroglia is upregulated in any sort of insult to the brain, be it inflammatory, hypoxic or traumatic - a reaction generally known as reactive astrogliosis (which can be considered an attempt to limit brain damage). ${ }^{48}$ Therefore, astrocytes play a dual role: depending on their state of activation by microglia, astrocytes display two distinct functional states, A1 and A2 - the latter having neuroprotective and anti-inflammatory properties, the former being highly neurotoxic and propagating inflammation. ${ }^{49}$ This Jekyll and Hyde quality seems to be a unique histological property of astrocytes (and also of microglial cells). This dual role may also be reflected in the fact that astrocytes are able to express huge amounts of Reactive Oxygen Species (ROS) scavengers and antioxidants but at the same time have the capacity for antioxidant neutralization through glutathione and ascorbic acid, as well as detoxifying enzymes. Given the role of astroglia in oxidative stress regulation and neuroprotection it is now generally accepted that astroglial performance to a large extent determines the outcome of brain pathology in a wide range of neurological damage. Indeed, astrocyte activation has been reported after CNS infection, trauma, ischemia, stroke, and also in autoimmune disease.

- Moreover, it has been shown that glial activation may also be a "mirror response" to systemic infections. ${ }^{3,50}$

- Also, astroglia takes part in modulating vagal tone and functioning: Astroglia of the nucleus of the solitary tract is responsible for integrating peripheral signaling that reaches the brain via the vagus nerve and thus takes part in modulating cardiovascular, respiratory, glucoregulatory, and gastrointestinal functions (including gastric emptying). ${ }^{51,52}$

- Moreover, neuroglia has been shown to influence exercise capacity, motor functioning and endurance. ${ }^{40,53}$ Microglia has been shown to be essential for protecting the brain from excessive activation, a functioning microglia may thus be a prerequisite for an adequate stress response. ${ }^{54}$

- Astrocytes intricately interact with the beta-2-receptor system. Beta-2-adrenergic receptors are heavily expressed on and in astrocytes and thus can affect neuronal functioning including cognition, memory, pain regulation and autonomous regulation. Indeed, the cognitive defects seen in patients with Multiple Sclerosis or Parkinson's and und Alzheimer's disease are now being understood as a consequence of $\beta_{2}$-receptor dysregulation in astrocytes. ${ }^{55}$

- Astrocytes are the main players in the brain Renin-Angiotensin-system (b-RAS), and are therefore potent regulators of cerebral blood flow especially in brain regions with high RAS activity - i.e. those regions associated with cardiovascular control. ${ }^{56-58}$

- Astrocytes and microglia also are essential components of the innate immune response through their ability to produce cytokines and chemokines, to express MHC-II molecules and to respond to pathogen-associated molecular patterns (PAMPS) and damage or dangerassociated molecular patterns (DAMPS) as well as inflammatory cytokines, chemokines and growth factors. ${ }^{45,59-64}$ Reactive astrocytes can both attract and corral immune cells and thus 
modulate inflammatory processes. Because activated astrocyte can also become antigen presenting cells their activation can extend to the induction of autoreactivity. ${ }^{65}$

- Also, NLRP3 expression has consistently been found in microglia and astrocytes, a finding relevant in the light of the fact that the NLRP3 inflammasome is now thought to be central in the initiation and propagation of neuroinflammation (for details, see 4.1).

- Neuroglia also intricately interacts with mast cells residing on the brain side of the blood brain barrier (the interaction of astrocytes and mast cells in inflammatory processes is indeed so close that the two have been called "partners in crime") (for details, see [4.5]). ${ }^{66}$

- Anatomically, glial cells are most dense along specific white matter tracts forming functional brain units by virtue of their capacity to activate other glial cells. ${ }^{3}$ These are in part independent of neuronal nuclei und clusters and also functionally open to a variety of transmitter systems. These intrinsic brain networks have a significant reach into distant brain locations, including the limbic and prefrontal areas of the brain and include and connect brain areas that have been implicated by different research groups in the pathology of ME/CFS: the basal ganglia/striatum, limbic structures (amygdala, hippocampus, thalamus, hypothalamus), brainstem/pons and neocortex as well as prefrontal cortex. Indeed, it is now understood that the glial networks are not only interwoven with the CNS neuronal circuits but are themselves regulators and determinants of neuronal function and synaptic interactions and thus integral part of the human connectome. ${ }^{67}$

In summary, astrocytes, often in cooperation with microglia, are involved in many regulatory functions including brain perfusion, neuroinflammation, innate immune responses and immune tolerance, the response of chemo- and baroreceptors, the modulation of the glutaminergic system and thus excitotoxicity, the regulation of the blood-brain-barrier and intracranial pressure as well as the regulation of peripheral inflammatory and other signals arriving through the vagal nerve. The astrocyte compartment has been shown to play a role in cognitive functions, memory retrieval, autonomous, psychomotor, cardiovascular regulation and the stress response. All these pathological and clinical features are recognized features of ME/CFS. Moreover, astrocytes and microglia play a dual role as anti-inflammatory protective players but at the same time can exacerbate brain pathology when severely stimulated.

Regarding the functions listed above there can be no doubt that this is a compartment which may be able to orchestrate many, if not all, of the core pathological features of ME/CFS.

In the following sections we will examine all relevant features of ME/CFS one by one and relate them to possible pathology in the neuroglial compartment.

\subsection{Evidence \#2:}

The clinical features of ME/CFS can all be explained as a consequence of neuroglial dysfunction

\section{$2.1 \quad$ Fatigue}


The fatigue in ME/CFS presents as a package of central (mental) and peripheral (muscular) fatigue which both seem to be closely related to an abnormal processing of the physiologic stress load (a connection that will be revisited, see [4.11]). Clinically, the fatigue seen in ME/CFS seems to be somewhat different from the typical "sickness fatigue" (sickness behavior) seen in many inflammatory diseases as it also includes manifestations of an overactivated arousal system (as clinically evidenced, for instance, by concomitant insomnia and sensory overstimulation).

Several hypotheses have been forwarded to explain central fatigue, including altered cytokine networks ${ }^{68}$ produced by immune activation (if peripherally, or in the CNS). It has indeed been shown that in chronic inflammation, cytokines may target the basal ganglia and dopamine function, producing persistent fatigue. ${ }^{69,70}$ Fatigue may also be a response to humoral and retrograde neural signals generated by inflammation elsewhere in the body..$^{50}$ There are now many indications that the most fundamental pathophysiological level of persistent fatigue may be neuroinflammation (for details, see [2.1]).

Indeed, activated micro- and astroglia has been shown to play a key role in mental fatigue ${ }^{42,71}$, and the astroglial compartment has been implicated in fatigue onset in several diseases including postviral syndromes like EBV infection ${ }^{72}$, chikungunya fever ${ }^{73}$, post-Ebola fatigue ${ }^{74}$ and, most recently, Long-Covid (for details see [8.0]).

The role of the micro- and astroglial compartment in the genesis of fatigue has also been well delineated for the fatigue seen in several non-infectious diseases:

- In Multiple Sclerosis, the severity of fatigue strongly correlates with the activation of microglial cells in the substantia nigra of the basal ganglia and the default mode network ${ }^{75}$ (a study reminiscent of Andrew Miller's study in ME/CFS patients ${ }^{14}$ which also linked reduced basal ganglia activation with fatigue, see below). Given the close cooperation of microglia and astrocytes this may explain why, in MS patients, the astroglial antigen glial fibrillary acidic protein (GFAP) correlates well with disease activity. ${ }^{76,77}$

- In Hepatitis $\mathrm{C}$, another disease associated with debilitating chronic fatigue, both fatigue and encephalopathic symptoms correlate with astrocyte dysfunction. ${ }^{78,79}$ Interestingly, here again, imaging studies have pointed to the basal ganglia.

- Several studies point to a central role glial activation in the often severe fatigue after traumatic brain injury (TBI), which also may explain the conspicuous overlap in symptomatology of TBI with ME/CFS ${ }^{80}$ (TBI is another disease in which the astroglial marker GFAP correlates with fatigue severity). ${ }^{81}$

- Similar correlations between fatigue and abnormalities in imaging studies have been seen in Gulf War Illness (GWI) ${ }^{82}$ and in fibromyalgia. ${ }^{83}$

What is known about fatigue and astroglial function in ME/CFS? Numerous anatomical and imaging studies in ME/CFS patients do support a possible link between fatigue and brain function (for a summary on neuroimaging results in ME/CFS, also see the section o neuroinflammation). Several imaging studies have related fatigue, cognitive function and reduced physical activity in ME/CFS patients to abnormalities in the cingulate cortex, the midbrain and basal ganglia ${ }^{84-86}$ as well as the brainstem..$^{87}$ For example, a functional MRI study comparing ME/CFS patients and controls, showed that mental fatigue, general fatigue as well as reduced activity in ME/CFS patients were correlated with decreased activation in the right globus pallidus ${ }^{14}$, a defined nucleus in the basal ganglia that had been implicated in chronic fatigue in several neurological disorders before ${ }^{88-94} \mathrm{~A}$ recent 
elaborate whole-brain Magnetic Resonance Spectroscopy study in ME/CFS patients related the functional changes in this nucleus to changes in the density or activation status of microglia and astrocytes. ${ }^{4}$ The latter results fit well with Barnden's findings of reduced white matter volume in the reticular activating system of ME/CFS patients - a region which closely cooperates with the basal ganglia ${ }^{95}$ and that has been implicated in the pathogenesis of fatigue before, both in humans ${ }^{96} 97$ and in animal models. ${ }^{98,99}$

Additional support for the link between astrocyte dysfunction and fatigue in ME/CFS comes from an experimental study in an animal model of post-infectious ME/CFS which describes evidence for a role for microglia and astrocytes in disease onset and maintenance. ${ }^{100}$ This has been replicated in a PET study of ME/CFS patients using the ligand (11)C-(R)-PK11195, a translocator protein that is expressed by activated microglia or astrocytes. ${ }^{101}$ The inflammatory glial response thus measured appeared to be in proportion to the severity of symptoms recorded, including fatigue.

In summary, the clinical phenomenon of fatigue indeed seems multiply linked to astroglial and possibly microglial dysfunction.

\subsection{Sleep problems and insomnia}

Sleep disturbances and insomnia are common in ME/CFS, with a pattern of restlessness and difficulty in falling and remaining sleep. ${ }^{102,103}$ Experimentally, it has been shown that the circadian rhythms are disrupted in ME/CFS. ${ }^{104}$

The circadian regulation is dependent on the molecular oscillations in the suprachiasmatic nucleus (SCN) which also plays an important role in the balance between the sympathetic and parasympathetic nervous systems, ${ }^{105}$ in the homeostatic regulation of the neural network mediating fatigue ${ }^{13}$ as well as in the firing rate of noradrenergic neurons of the locus coeruleus, the main source of norepinephrine release. ${ }^{106}$ Dysfunctions of the suprachiasmatic nucleus (and of the paraventricular nucleus to which it is intensely connected) have understandably been implied in several hypotheses of $\mathrm{ME} / \mathrm{CFS}$ pathology. ${ }^{7,104}$

The circadian function of the SCN has been shown to be driven by resident astrocytes. ${ }^{107}$ Also, the function of the SCN has been shown to be heavily influenced by pro-inflammatory processes; these influences are mediated through activation of resident microglia and astrocytes. ${ }^{108,109}$

With this close interplay of the innate immune system, astrocyte function and the SCN it appears plausible that the neuroglial compartment may play a role in the sleep disturbances observed in ME/CFS.

\subsection{Cognitive and memory impairment}


Disturbed cognition (including difficulties with word retrieval and remembering) is one the core symptoms of ME/CFS and highly associated with severe levels of fatigue and sensory hyperreactivity. ${ }^{12}$ Imaging studies in ME/CFS patients have shown multiple alterations in the prefrontal cortex, combined with neuroinflammation in the midbrain. ${ }^{110,111}$

Experimental evidence suggests that the neuroglial compartment is centrally involved in cognitive and memory dysfunctions. ${ }^{112}$ Astrocytes seem to especially contribute to memory consolidation and seem to mediate the effects of vigilance and arousal on memory performance. ${ }^{113}$ These effects are now being understood as a consequence of $\beta_{2}$-receptor dysregulation in astrocytes (beta-2receptors are heavily expressed on astrocytes). ${ }^{55,114}$ This link to the adrenergic system may explain the decrease of cognitive functions with stress typically also seen in ME/CFS. ${ }^{115}$ It has also been suggested that cognitive dysfunction may result from overstimulation of NMDA receptors by glutamate when dysfunctional astrocytes lose their ability to scavenge enough glutamate. ${ }^{116}$

In summary, memory and cognitive functions seem to be tied to proper astrocyte functioning. In the light of widespread neuroinflammation in the midbrain and prefrontal cortex in ME/CFS patients it may be plausible that astrocyte dysfunction may contribute to the cognitive alterations seen in ME/CFS.

\subsection{Sensory hyperreactivity}

Hypersensitivity to light and sounds as well as sensory stimuli (like rapidly changing stimuli) is a very typical symptom in ME/CFS and also noteworthy because it is quite rare in other diseases. An analysis of the more severely affected ME/CFS patients has shown that sensory hyperreactivity indeed belongs to the most discriminatory symptoms of ME/CFS (together with cognitive dysfunction, severe fatigue and severely reduced exercise capacity). ${ }^{12}$ In experimental studies sensory hyperreactivity seems to correlate well with autonomous dysfunction and adrenergic hyperstimulation.

From a basic research angle this s not surprising. Sensory overstimulation has been linked to glutamate dependent excitotoxicity. Indeed, sensory gating has been shown to be a glutamate dependent process and here the astrocyte compartment obviously plays the central role as astrocytes by large determine glutamate availability in the brain. ${ }^{117,118}$ This goes well with the clinical link noted above: in ME/CFS, cognitive impairment and sensory overstimulation go hand in hand. This fits with the observation that damage to astrocytes causes an overstimulation of NMDA receptors which in turn may be responsible for changes in cognitive function in the frontal cortex. ${ }^{116}$

In summary, it is plausible that the sensory hyperreactivity observed in ME/CFS may be related to astrocyte dysfunction.

\subsection{Psychomotor slowing and the "coupled" nature of ME/CFS}


As described in the introduction, central and peripheral clinical manifestations of ME/CFS go hand in hand. This "packaging" can be explained in two ways. Either ME/CFS is based on a general pathological process that affects central and peripheral functions at the same time. Candidate processes for such a "coupled" pathophysiology may, for instance, be general cellular dysfunctions as well as general endothelial dysfunction/altered vascular perfusion. Alternatively, the clinical picture of ME/CFS may reflect a regulatory disorder that primarily affects regulatory centers in the CNS which then may secondarily disturb peripheral functions.

Psychomotor slowing, i.e. the observation that cognitive-mental dysfunction and motor dysfunction go hand in hand, seems to be a case in point. There are indeed many hints that motor slowing in $\mathrm{ME} / \mathrm{CFS}$ may be a reflection of "central" dysregulation:

- While muscular fatigue can indeed originate on different levels of the motor pathway and can theoretically be related to central and peripheral components, any level of muscular fatigue can clearly happen from central dysregulation alone; muscular fatigue emanating from central pathology has been shown to be related to abnormalities in deep brain circuits involved in motor planning and execution, i.e., in a decreased neural drive to the muscle. ${ }^{119-}$ 121

- For ME/CFS, there is now indeed ample evidence that the motor dysfunction is mediated by a reduction in the ability of the CNS to generate motor neurotransmission. ${ }^{122-125}$

- Clinically, motor slowing in ME/CFS very consistently correlates with mental fatigue (see above). The correlation between fatigue and motor slowing may even have some anatomical-topographical correlation as both fatigue and motor slowing have been associated with basal ganglia dysfunction (and, specifically, seem to both correlate with reduced activation of the striatum). ${ }^{14}$

- Conspicuously, motor slowing during a ME/CFS exacerbation seems to be clinically different from motor slowing following regular muscular exhaustion as it includes phenomena reminiscent of neurological compromise: not only is muscular strength reduced during PEM, but also gait regulation seems to be affected with individuals unable to perform normal strides and maintain effective coordination.

- Similar psychomotor pictures associated with fatigue as seen in ME/CFS (i.e. fatigue, which extends to executive functions but also to "reward" circuits) is typically seen by neurologists in disorders with lesions affecting the attention and arousal system, or with lesions in the reticular activating and limbic system, or with lesions in the basal ganglia. ${ }^{94}$ This "mental" type of "psychomotor" fatigue is also well described in MS, and here the same nuclei and regulatory centers have been implicated as in ME/CFS. ${ }^{75}$ Similar correlations are seen in hepatitis $C$, which often presents as encephalopathy with a striking clinical overlap with $\mathrm{ME} / \mathrm{CFS}$. Here again similar brain structures may be involved. ${ }^{126}$

What role may the neuroglial compartment play in motor slowing and muscular fatigue in ME/CFS? According to experimental findings both peripheral and central inflammation are closely linked to motor drive, abilities and activity. Given the central role that glial activation plays in neuroinflammation, a role of astrocytes in this psycho-motor-immune picture appears plausible. ${ }^{47,127}$ Indeed, it could be shown that astrocytic IL-6 may mediate locomotor activity and exploration behavior in general. ${ }^{128}$ Also, astrocytes seem to be involved in the dopaminergic "reward" system which has been implicated in motor control and regulation. ${ }^{129}$ 
In summary, there is reasonable evidence both from clinical observations and pathophysiology which points toward a central "lead" in the pathological process of ME/CFS (which does not preclude secondary ubiquitous dysfunction or pathology). The phenomenon of psychomotor slowing, so prominent in ME/CFS, may plausibly be a reflection of astrocyte dysfunction.

\subsection{Mood issues, including anxiety and depression}

One of the most vicious aspects of ME/CFS is the frequent admixture of anxiety, panic and depression, especially during exacerbations and PEM. These mood disturbances, in conjunction with the frequent feeling of unease and agitation, may be responsible for the "death like quality" that many ME/CFS sufferers report. The profoundly anhedonic picture of ME/CFS has also been related to a dysfunctional reward system.

There is evidence from many angles that the astroglial compartment is at the center of mood regulation and also may play a role in the modulation of the reward system:

- in models of depression and anxiety disorders the excitatory-inhibitory imbalance caused by astrocyte dysfunction has been implicated as an important pathogenetic factor. ${ }^{130}$ Indeed, there is now clear and ample evidence for a role of glia in neuropsychiatric disorders (for a review see ${ }^{131}$; for details on possible cellular mechanisms see ${ }^{130}$ ).

- astrocytes seem to be heavily involved in the dopaminergic "reward" system. ${ }^{129}$ For instance, astrocytes influence synaptic functions in several reward circuits. ${ }^{132}$

- in animal models, astrocytic IL- 6 has been shown to mediate anxiety. ${ }^{128}$

So taken these pieces of evidence there is no doubt that the profound "psychiatric" aspects of ME/CFS may be related to astroglial dysfunction.

\subsection{Gastrointestinal symptoms}

Many ME/CFS patients report gastrointestinal symptoms including indigestions, esp. fullness and bloating after meals, abdominal distension, nausea, and loss of appetite. Lower gastrointestinal tract symptoms are also common and show considerable overlap with irritable bowel syndrome. ${ }^{133}$

Also, changes in the intestinal microbiome have been noted in $\mathrm{ME} / \mathrm{CFS},{ }^{134,135}$ possibly giving rise to translocation of bacteria across mucosal barriers. ${ }^{136}$ It appears that the dysbiosis in ME/CFS is not just an epiphenomenon but may reflect gastrointestinal inflammation which in turn may have immunologic/inflammatory ramifications ${ }^{137,138}$ (for a thoughtful commentary, see ${ }^{139}$ ). It is now understood that microbial metabolites have multiple roles in host physiology und may have far reaching impact on host immune, metabolic and hormonal signaling and thus may also influence CNS regulatory functions. ${ }^{140}$ For instance, it has been shown that metabolites derived from dietary tryptophan crossing the BBB may affect NF-KB signaling (a pathway for innate and adaptive immune system activation which has also been implicated in ME/CFS) and activate astroglial cells. ${ }^{141}$ 
As to the upper gastrointestinal problems it appears plausible that they may be related to dysmotility due to the widespread autonomous dysfunction seen in ME/CFS. This is corroborated by the finding of slow gastric emptying in ME/CFS patients. ${ }^{142}$ This finding may point to a role of the nucleus of the solitary tract (NTS) in the brainstem which contributes both to the control of cardiovascular, respiratory, glucoregulatory, and gastrointestinal functions, including gastric motility. Physiologically, the NTS is heavily dependent on a regular vagal input, which in turn is dependent on a functional astroglial compartment (for details, see [4.10]).

So, while not well delineated, a role of the astroglial compartment in the gastrointestinal dysregulation seen in ME/CFS appears plausible.

\subsection{Evidence \# 3}

\section{The core feature of ME/CFS, post exertional malaise, can be explained as a consequence of neuroglial dysfunction}

Post-exertional malaise can be best described as a disease exacerbation triggered by exercise. Indeed, PEM is nothing else than ME/CFS written large: pretty much the same symptoms, the same dysfunctions - only more (and often, much more) severe. This clinical nature of PEM may be noteworthy for hypothesis generation about ME/CFS in general: whoever is able to explain PEM may be able to explain ME/CFS (this is the reason why this section is more detailed than others).

Post-exertional malaise unites a few noteworthy features: ${ }^{143}$

- while it can be induced by muscular activity it can clearly also occur independently of peripheral muscular or cardiovascular challenges, e.g. as a consequence of emotional, mental, cognitive or sensory stimuli

- it clearly follows an individually calibrated threshold dynamic which seems to correlate with disease activity: the milder affected ME/CFS patients have a higher PEM threshold than the more severely affected. This dynamic is also seen in individual patients: if the functional level is already low when the overexertion happens, PEM is more likely to happen at a lesser stress level and to be more severe

- also, there seems to be a dose-response dynamic: the more severe the overexertion, the more profound and longer PEM will be

- the clinical deterioration of PEM starts within a few hours to about a day after the triggering event (the latency may also be dependent on the nature of the exercise, with PEM caused by cognitive overload possibly having a shorter latency), peaks in severity 72 hours after strenuous exercise and lasts from a few days to several weeks, depending on baseline functionality and degree of exercise.

- Clinically, PEM is typically accompanied by symptoms of immune stimulation like lymph node swelling and flue-like symptoms.

Many authors have hypothesized an "inflammatory" pathogenetic picture behind the phenomenon of PEM in ME/CFS, too. Yet, the exact mechanism remains a matter of debate. Exercise has been 
suggested to trigger metabolic or mitochondrial derangements in the muscle cells of ME/CFS patients (or possibly in the cells in general), which in turn may lead to diminished blood flow and general misery perfusion. Also, exercise may trigger endothelial dysfunction directly, e.g. by activating dysfunctional beta-2-receptors (incapacitated by autoimmune processes, for instance). Likewise, exercise could alter the gut microbiome and provide for a temporary increase in gut permeability which in turn may send inflammatory signals related to "leaked" bacteria or bacterial products. ${ }^{144}$ These processes could either cause dysfunction directly or activate general inflammatory pathways and/or neuroinflammation.

While the final jury is still out if PEM represents "peripheral", general, or "central" pathology there is some biomechanical plausibility in support of the hypothesis that PEM may be related to tonic activation of neuroinflammation.

Here, we need to briefly revisit the role of astroglia (for details, see [1.0]). The astroglial and microglial compartment is noteworthy for its plastic response to endogenous stimuli. Indeed, astrocytes and microglia have been shown to possess a duality (or, probably multiplicity) in their phenotype, which can shift between "neuroprotective" and "neurotoxic" properties, depending on stimuli, and physiological/pathological condition. ${ }^{145-147}$ As a consequence of activation by various stimuli, microglial cells and astrocytes shift to a pro-inflammatory phenotype, which increases the release of cytokines, chemokines, and neurotoxic factors thus promoting immune stimulation, neuroinflammation and CNS dysfunction. ${ }^{148,149}$ This activation has been shown to be able to foster a self-perpetuating hyper-response: previous encounters of inflammatory stimuli will "prime" the glial compartment for an exaggerated response to subsequent challenges. As experiments have shown, this exaggerated response can emanate from stimuli of similar nature (i.e. mediated through microbial antigens) but may also be of a different kind (including psychological stress). ${ }^{150,151}$ From a basic biological understanding it is therefore plausible that stress signals of many kinds may switch astrocytes and microglia to a proinflammatory phenotype, causing astroglial malfunction with a wider range of associated CNS dysfunctions.

This tonic reaction of the glial compartment to stress signals may explain the latency dynamic of PEM. The delay in onset of symptoms after exercise in ME/CFS may correspond to the time frame needed for any pro-inflammatory signals (like cytokines, see below) to a) reach the brain and b) induce glial "priming". Possibly, the duration of PEM may correspond to the time frame of astrocytes and microglia to revert to a more quiescent stage after being activated. The individually calibrated PEM threshhold may correspond to the individual reactivity of the neuroglial cells involved, i.e. the degree of "priming". The exercerbation of symptoms during PEM may reflect CNS dysfunction caused by propagation of glial dysfunction into distant brain areas and regulatory centers along glial functional networks.

The experimental record may fit with the hypothesis of a pathological glial activation from exercise:

- Basic research shows that the biological effects of exercise may be completely different in healthy people than in patients suffering from inflammatory conditions, including infections and autoimmune diseases: While exercise may lead to a balanced proinflammatory and antiinflammatory response in healthy people, it may lead to an unbalanced and exaggerated inflammatory response in patients with pre-existing inflammatory conditions. ${ }^{152}$ 
- In a psychosocial stress test, those ME/CFS patients with greater baseline inflammation level (as measured by LPS-induced IL-6 and TNF-alpha levels) had higher mental and physical fatigue scores after the stress test. ${ }^{153}$ This may fit with the findings in a gene expression study of physical exercise in ME/CFS patients that any gene expression in response to exercise was stimulated much earlier in ME/CFS patients than in normal subjects. ${ }^{154}$

- In a study of physical activity in ME/CFS patients, an increase of the pro-inflammatory cytokines IL- 6 and IL-1 beta in the blood at 8 hours after the exercise was predictive of higher fatigue scores after the exercise ${ }^{155}$ (IL6 and IL1- $\beta$ are both known for their role in glial activation). This pro-inflammatory milieu may also be reflected in a higher and prolonged oxidative stress response in ME/CFS patients after exercise ${ }^{156}$ as well as an exercise-triggered activation of the complement cascade, including increased levels of Mannose-binding lectinassociated serine protease-2 (MASP-2) and of C4a (which may play a role in glial activation). 157158

- Leukocytes in ME/CFS patients have been shown to react with brisk TLR-4 expression in response to exercise ${ }^{159}$ (TLR4 is an activator of the innate immune system and can mediate microglial activation and neuroinflammation) $)^{47,160,161}$

- Another cytokine profiling study in ME/CFS patients during submaximal exercise identified the cytokine CXCL10 as a central player in the cytokine network post exercise. ${ }^{162}$ This signalling molecule is expressed on glial cells and may play an important role in the amplification of neuroinflammation. ${ }^{163}$

- In another study, mitochondrial DNA associated with exosomes has been shwon to be elevated in ME/CFS patients after exercise. ${ }^{164}$ At the same time, this team has shown that exosomes isolated from patients with ME/CFS stimulate secretion of IL-1 $\beta$ from cultured human microglia.

- The conspicious finding in ME/CFS that PEM may be triggered both by physical and by cognitive exercise may be explained from a functional MRI study of ME/CFS patients, in which symptom exacerbation after both mental and physical exercise correlated with neurophysiological effects on brain function. ${ }^{165} 166$

- The most informative study of PEM in ME/CFS, however, may be an experimental study by Light et al. who used gene expression microarrays to find genes that may be over- or under expressed 8, 24 and 48 hours after exercise in ME/CFS patients compared to healthy controls. ${ }^{154}$ Their gene expression results (which correlated well with fatigue levels postexercise) may again suggest a profound "neuroimmune" nature of PEM (most of the gene expression markers identified in this study have only recently been functionally described, which may explain why the authors have interpreted their findings differently). Indeed, the findings of increased expression of ASIC3, P2X4, P2X5, a-2A, b-1, b-2, COMT, IL10, TLR4 and possibly TRPV1 in ME/CFS patients after exercise provides plausible evidence that PEM may be pathologically based on inflammatory hyperstimulation, adrenergic dysregulation (overexpression of a-2A, b-1, b-2 and COMT genes), activation of the innate immune system (overexpression of TLR4 genes as well as IL1-beta), and activation of the microglial and astroglial compartment suggesting a role of neuroinflammation (both TRPV1 and the P2X ion channels are expressed on glial cells and play a role in neuroinflammation). ${ }^{167,168159169}$

So, the question would be how the astroglial compartment may be activated through exercise stimuli. There are several options which may possibly in part be interlinked: 
- The role of cytokines (for a review, see ${ }^{170}$ and ${ }^{171}$ ): Pro-inflammatory cytokines (esp. IL 6) have been shown to be heavily induced by exercise in healthy people, ${ }^{154,172,173}$ with levels increasing up to 100 fold with maximal exertion. The source of the interleukin production from physical exercise is thought to be mostly the muscles. The magnitude of the exerciseinduced IL-6 response seems to be dependent on the intensity and especially the duration of the exercise. In high intensity exercise IL-1-beta is also increased. The interleukin response to exercise seems to be independent of the nature of the exercise. ${ }^{174}$ Indeed, it could be shown that psychological or mental stress alone can also induce cytokine release, ${ }^{175}$ including IL-6 and IL-1-beta, with a $>6$-fold increase over baseline. ${ }^{162,176}$ Activity-induced activation of neuroinflammation in ME/CFS appears plausible as several of the cytokines normally induced by activity (like IL- 6 and IL-1 $\beta$ ) have been shown to be able of glial activation. ${ }^{4-46}$

- The role of cerebral hypoperfusion: Cerebral perfusion has been shown to be dysregulated in ME/CFS patients (for details, see below), and this may be an important link in the pathogenesis of PEM, too. Any stress exposure, be it physical or mental, emotional, sensory or cognitive, places increasing demands on brain perfusion which in ME/CFS patients may not be adequately matched to physiologic requirements. Therefore, any allostatic load exposure exceeding the regulatory capacitiy of the cerebral blood circulation may cause a state of profound cerebral hypoxia and energy depletion. This may directly induce cerebral dysfunction, or may indirectly lead to cerebral functional impairment, e.g. by inducing reperfusion injury, lactate elevation, blood-brain-barrier dysruption, cerebral edema, ROSproduction, activation of the NLRP3-inflammasome or other processes that may induce glial reactivity and aggravate neuroinflammation (for details, see next section).

- The role of GPC-receptor dysfunction: It is also possible that some effects related to exercise (e.g., increased norepinephrine levels) may, in ME/CFS patients, induce increased G Protein Coupled Receptor (GPCR) dysfunction. A variety of autoantibodies against GPCR, including against alpha and beta adrenergic receptors have been found in ME/CFS patients and may contribute to dysfunctional singnalling not only in the peripheral vasculature but also on several regulatory levels in the CNS. Here it may have to be remembered that adrenergic receptors span a wide range of effector and regulatory functions both in the periphery and in the brain (the majority of adrenergic receptors are expressed in the brain, especially in the brain regions involved in autonomic activity and cardiovascular regulation). ${ }^{177} 19035 \mathrm{In}$ the brain, especially $\beta 2$-adrenergic receptors are widely expressed both on glial, endothelial and immune cells which are therefore activated in response to norepinephrine release. ${ }^{178}$ Adrenergic receptors have been implicated in the regulation of arousal, in the regulation of cognition and memory, ${ }^{112,115}$ but also in the regulation of the immune system. It has indeed been shown that physiologic beta-2-adrenergic signalling is involved in maintaining immune tolerance, ${ }^{179}$ controlling inflammatory input through the vagus nerve ${ }^{180}$ and in controlling neuroinflammation. ${ }^{181,182}{ }^{183}$ Dysfunctional beta-2 signalling may therefore have significant infuences not only on autonomous regulation but also on the inflammatory response (and other immune responses).

- It has also been suggested that exercise could provide for a temporary increase in gut permeability which in turn may send pro-inflammatory signals related to "leaked" bacteria or bacterial products (the latter may then induce neuroinflammation through the gut-brain axis). ${ }^{144}$ 
In summary, while much has still to be learned about the pathobiological basis of PEM there is plausible evidence to conceptualize PEM as a dysfunctional, inflammatory stress response mediated by the neuroglial compartment.

\subsection{Evidence \#4}

\section{The pathological features of ME/CFS can be explained as a consequence of neuroglial dysfunction}

We have discussed the clinical features of ME/CFS and how they may be explained as a consequence of astrocyte dysfunction. We will now examine the patholophysiological features of ME/CFS one by one to ask the same question: Can they also be explained by assuming astrocyte dysfunction as the central hub in ME/CFS?

\subsection{Neuroinflammation}

The term neuroinflammation is still under definition among neuroimmunologists and somewhat contentious outside the laboratory, in part be owed to the fact that neuroglial activation or inflammatory processes in the CNS are difficult to assess in humans due to the inaccessibility of the affected tissues for biopsies; also, the restricted and selective trafficing of inflammatory mediators, cellular markers and immune cells across the blood brain barrier and into the cerebrospinal fluid limits the diagnosis of neuroinflammation through blood work or spinal tap.

The term neuroinflammation denotes a wide range of immune responses in the central nervous system, including spinal cord (for a review, see ${ }^{184}$ ). The neuroinflammatory responses are mainly orchestrated by astroglia, interacting with microglia, microvascular endothelial cells and peripherally derived immune cells. ${ }^{185,186}$ The involvement of astroglia - which in particular is able of inflammatory propagation along the white matter tracts within the CNS but also into the periphery - may explain the broad, multilevel clinical effects of neuroinflammation.

Neuroinflammation leads to the release of inflammatory mediators and signaling molecules (including extracellular ATP, excitatory glutamate and nerve growth factor), increased vascular permeability, blood-brain barrier disruption, endothelial activation and local changes of perfusion, leucocyte infiltration and mitochondrial dysfunction (for details on the latter, see [4.7]). On the functional level, neuroinflammation has been associated with cytokine-mediated sickness behavior, excitotoxicity, pathological recruitment of regulatory nuclei and/or disruption of connectivity within the brain laying the ground for "downstream" functional CNS disruptions extending to cognition, memory, pain regulation, autonomous regulation etc.

Neuroinflammation is also notable for several "amplifying" effects: 
- chronic or repetitive neuroinflammation may cause autoimmune reactions (or trigger preexisting autoimmunity) to all sorts of components of the brain circuitry - a common feature in many diseases involving neuroinflammation. ${ }^{187,188}$

- neuroinflammation may stimulate systemic inflammation (for details on this noteworthy phenomenon which includes vagal brain-body immune signaling, see [4.10]). ${ }^{189} 190$

- chronic neuroinflammation can especially induce the vicious circle of microglial priming, i.e. the induction of hyperresponsiveness to any further stimulation, resulting in a selfpropelling cycle of neuroinflammation (for details, see [1.0]).

The exact cause of neuroinflammation often remains unknown. Possible causative mechanisms include direct effects through injury or infection of the brain, reactivation of endogenous microbial reservoirs in CNS cells (like EBV and other herpesviruses), ${ }^{10,191,192}$ autoimmune processes against specific neural or immune system targets, repetitive mechanical strain (for details, see [5.0]), cerebral hypoperfusion, primary vagal dysfunction, high concentrations of glutamate or norepinephrine overload (for details, see[4.11]).

Remarkably, neuroinflammation can also occur in response to humoral and retrograde neural signals generated by inflammation elsewhere in the body, a process that may be very noteworthy for the understanding of a postinfectious neuroimmune phenomenon like ME/CFS. ${ }^{50,190,193}$ Indeed, several studies have shown that systemic injection of cytokines like IL-1 beta, IL- 6 or TNF-alpha alone is sufficient to cause glial activation and thus inflammatory changes in the brain. Also, it has been shown that peripheral inflammation can result in inflammatory cytokines remaining elevated in the brain for at least 10 months. ${ }^{194}$ This inflammatory "mirror response" can be triggered through afferent neuroimmune signaling by the vagal nerve (for details, see [4.10]) or by cytokines being actively transported across the BBB (if systemically present in high enough concentrations, cytokines can also passively diffuse through the BBB via circumventricular organs).

From pathphysiological considerations it appears plausible that neuroinflammation may be responsible for the diverse symptoms of ME/CFS like fatigue, autonomous dysfunction, sensory hyperreactivity, alterations in mood, memory and cognition etc - and may also explain why symptoms in ME/CFS wax and wane in response to endogenous or exogenous stress stimuli . Yet, there is only limited direct evidence of neuroinflammation in ME/CFS so far. After all, neuroinflammation is not easy to "diagnose" given the limitations presented above. The analysis of cytokines in the CNS, for instance, is difficult to interpret for methodological reasons. ${ }^{190}$ Several studies in ME/CFS patients have suggested elevated levels of pro-inflammatory cytokines as well as decreased anti-inflammatory cytokines in the cerebrospinal fluid, ${ }^{195-197}$ yet, it has been argued that this may not be a reliable marker for neuroinflammation. ${ }^{190}$ Established and convenient blood markers for astroglial integrity like Glial Fibrillary Acidic Protein (GFAP) have so far - and surprisingly not been investigated in ME/CFS. Corroborating evidence for astrocyte activation in ME/CFS may be gleaned from a sibling disease, Gulf War Illness, where anti-GFAP autoantibodies are consistently present (here again GFAP has not yet been measured directly). ${ }^{198}$ Recently it has been shown that the complement factor C1q seems to be elevated in a subset of ME/CFS patients (with some correlation with disease severity). ${ }^{199}$ While this could generally point to a stimulated innate immune system, this may also indicate neuroinflammation, after all, microglia seems to be the dominant source of $\mathrm{C} 1 \mathrm{q}$ (at least in the mouse brain). ${ }^{200} \mathrm{C} 1 \mathrm{q}$ has been shown to be "dramatically" upregulated in brain microglia in response to ischemia ${ }^{201}$ and seems to play a central role in neurodegenerative and neuroinflammatory diseases in humans. ${ }^{202}$ 
Corroberative evidence of neuroinflammation in ME/CFS comes from PET studies (see below), from magnetic resonance spectroscopy (MRS) studies which show increased levels of several metabolites related to neuroinflammation, ${ }^{4,203-205}{ }^{206}$ _and from other imaging studies which detected elevated levels of lactate in the cerebral ventricles of ME/CFS patients. ${ }^{207-211}$ (For a summary of imaging studies in ME/CFS, see ${ }^{110}$ ). Direct evidence for a central role of astroglial dysfunction in $\mathrm{ME} / \mathrm{CFS}^{95}$ is provided by a PET study using the translocator protein TSPO which specifically labels microglia and/or astrocytes. ${ }^{101}$ In the latter work the imaging findings were also associated with the severity of neuropsychologic symptoms of ME/CFS including fatigue sensation, cognitive impairment, pain, and depression. Indirect evidence for astroglial involvement in ME/CFS can also be gleaned from the findings of impaired intra-brainstem connectivity, ${ }^{5}$ from the findings of impaired glutamate levels in key regulatory brain areas ${ }^{212,213}$ from the findings of increased choline signaling in the basal ganglia of $\mathrm{ME} / \mathrm{CFS}$ patients which is thought to indicate increased cell membrane turnover due reparative astrogliosis ${ }^{205}$ or from a reduction myo-inositol which may be consistent with glial dysfunction. ${ }^{206}$ These findings have been expanded by experimental work on an animal model of ME/CFS by Mami Noda et al, who has identified molecular mechanisms through which microglia and astrocytes may trigger or maintain immunologically induced ME/CFS. ${ }^{100}$ There is now also good evidence how astroglial activation and neuroinflammation may be tied to the central clinical phenomenon of $\mathrm{ME} / \mathrm{CFS}$, post-exertional malaise (for details, see [3.0]).

In summary, a central role for neuroinflammation in ME/CFS seems plausible and several links to glial (dys)function have been delineated.

\section{Neuroinflammation and the role of the NLRP3 inflammasome}

Recently it has been proposed that the neuroinflammation seen in ME/CFS may be mediated by a complex of proteins known as the nucleotide-binding oligomerization domain-like receptor pyrin domain-containing-3 inflammasome (in short, the NLRP3 inflammasome). ${ }^{214}$ NLRP3 activity has also received interest in other neuroinflammatory disorders in which astroglial involvement may play a role like Alzheimer's disease, stroke, traumatic brain injury, Parkinson's disease or MS. ${ }^{215}$ It is thought that NLRP3 activation may reflect the activation of the innate immune system, which has been suggested as a central pathogenetic feature in ME/CFS. Interestingly, while the expression of NLRP3 in neurons has been discussed controversially, expression of NLRP3 has consistently been found in microglia and astrocytes.

The emerging role of the NLRP3 inflammasome and its activation through pathogen-associated molecular pattern molecules (PAMPs) as well as damage-associated molecular pattern (DAMPs, including reactive oxygen species, heat shock proteins or extracellular matrix molecules) may explain the well-established link between immune activation and fatigue, a link which may indeed involve the astroglial compartment (for details, see [4.4. ${ }^{215}$ The link between the presence of DAMP/PAMPs and the activation of the NLRP3 inflammasome may also explain why the presence of microbial remnants ("viral ghosts") or the inflammation-induced reactivation of endogenous viruses or other microbes may lead to chronic neuroinflammation ${ }^{216,217}$ (one of the hypotheses how ME/CFS may be triggered, see [7.0]). 
Interestingly, the link between the activated innate immune system and astroglial activation has been shown to include signaling through IL-1 $\beta$ and the JAK/STAT3 pathway. ${ }^{218}$ Both have been implicated in studies of ME/CFS patients. For example, a recent micro RNA study of ME/CFS patients found dysregulated miR-223, a micro-RNA that modulates the TLR4/TLR2/NF-KB/STAT3 signaling pathway and has also been shown to inhibit the NLRP3 inflammasome. ${ }^{219}$ Similarly, and also of possible interest, vagal input may also inhibit the NLRP3 Inflammasome using similar signaling pathways. $^{220}$

The above associations may explain the emerging interest in the NLRP3 inflammasome in ME/CFS research; after all, the question through which route stress signals may cause the profound neuroimmune resonance seen in ME/CFS remains unanswered.

\subsection{Cardiovascular dysfunction}

$\mathrm{ME} / \mathrm{CFS}$ is associated with a complex cardiovascular situation marked by low blood volume, reduced preload, low stroke volume, chronotropic incompetence, paradoxically low RAAS activity with possibly very high Angiotensin II levels, intermittent fluid retention, compromised microcirculation, endothelial dysfunction and clinical signs and symptoms of orthostatic intolerance (often with normal heart rate and blood pressure changes; Postural Tachycardia Syndrome/POTS however seems to be rare in ME/CFS patients). ${ }^{2,20}$

There are two somewhat competing, but possibly interlinked explanations for this complex set of dysfunctions. One explanation starts from the assumption of a general regulatory defect in the vasculature. Dysfunctional GPC-receptor signaling including pervasive adrenergic, and especially beta-2-dysfunction, as well as capillary dysregulation may reflect or drive this picture, as thoroughly described by the Wirth/Scheibenbogen hypothesis. ${ }^{2}$

However, there are reasons why the circulatory compromise seen in ME/CFS may also be explainable by a central regulatory dysfunction rather than as a general systemic dysfunction:

- Clinically, in ME/CFS patients, the "cardiovascular" symptomatology is always coupled with "encephalopathic" symptomatology (for details, also see [2.5]). Indeed, it has been shown that markers of cardiovascular dysregulation like decreased heart rate variability correlate well with symptoms of cognitive impairment in patients with ME/CFS. ${ }^{221}$ This synchronicity may of course also be explainable by a general (systemic) vascular dysfunction which also extends to the brain - yet, so far, the mechanism for this body-brain connection remains unexplained (the components of the peripheral RAAS - identified as dysfunctional in ME/CFS - cannot access most brain regions because of the blood-brain barrier). ${ }^{222}$

- Also, severe cardiovascular deterioration can be observed after exclusively cognitive or exclusively mental exertion alone (i.e., without any muscular activity).

- It has been shown that central autonomic cardiac control centers may indeed be dysfunctional in ME/CFS (for details, see [4.10]).

- Also, a "central" explanation may be able to explain why cardiovascular deterioration in phases of PEM so typically happens after a specific time window of 12-48 hours after any type of exertion, be it physical, cognitive or emotional (for details, see [3.0]). 
Which central processes may ultimately explain the enigmatic cardiovascular situation in ME/CFS? Here the lead may start from the speculation that in ME/CFS, in spite of paradoxically low Renin levels, Angiotensin II levels may be high (this is clearly a speculation as Ang II levels have so far only measured in POTS but not in ME/CFS - in POTS they are very high). ${ }^{223,224}$ Effects of angiotensin II include norepinephrine release, i.e., a ramped-up flight/fight system, narrowed blood vessels, low blood volume and reduced blood flow to the brain. Reasons for the suggested high angiotensin II levels remain unknown, speculations include low ACE-2 levels (e.g. from a dysfunctional ACE-2 receptor) as well as desensitization or autoreactive changes in Angiotensin II receptors.

The abnormal cardiovascular situation in ME/CFS is often attributed to a dysregulated peripheral ("classical") RAAS. However, angiotensin also takes center stage in an important central regulatory unit, the brain Renin-Angiotensin-system (brain RAS = b-RAS, see also [3.0]). Indeed, all RAS components are present in the brain and heavily enriched in cardiovascular regulatory CNSregions. ${ }^{56-58}$ Astrocytes in these regions are the main source of angiotensinogen and also heavily express Ang II receptors. ${ }^{149}$ At the same time, angiotensin II also acts as a central neurotransmitter.

Indeed, the brain RAS has been shown to be able to impact autonomous cardiovascular regulation via several mechanisms, including

- the modulation of central autonomic regulation through altered neurotransmission ${ }^{225}$ which in turn may influence the central sympathetic outflow to the periphery. ${ }^{226,227}$

- the facilitation of a glial cell-dependent proinflammatory milieu within cardiovascular control centers $^{228}$

- a tight influence on the kallikrein-kinin system (incl. bradykinin and its receptors) with expected effects on vascular function and tissue perfusion ${ }^{229}$

- the modulation of cardiovascular reflexes via changes in baroreflex sensitivity (e.g. via angiotensin II type 1 receptors (AT1R) in the Nucleus of the solitary tract (NTS). ${ }^{230,231}$ As a consequence of the diminished baroreflex sensitivity, changes in blood pressure may not correlate with adequate changes in brain perfusion any more (for details on baroreceptor changes in ME/CFS, see [4.8]).

- How profound these effects may be can be gleaned from the fact that abnormal central angiotensin II signaling has been shown to correlate with oxidative stress, endothelial dysfunction, BBB breakdown, neuroinflammation and hypoperfusion ${ }^{232}$ (all well known parts of the pathological matrix of ME/CFS).

So, clearly, the effects of a possibly dysregulated brain-RAS-axis not only include peripheral effects but also pro-inflammatory effects and altered cardiovascular regulation in the brain.

Interestingly, the hypothesis that the cardiovascular dysregulation in ME/CFS may be tied to a dysfunctional brain-RAS may also be able to explain the intricate correlation between cardiovascular and "central" symptoms like cognitive dysfunction (for a review, see ${ }^{232}$ and $^{233}$ ). Indeed, it has been shown that over-activation of $\mathrm{AT}_{1}$ Receptors on astrocytes correlates with cognitive impairment ${ }^{222,234}$ and changes in mood. Not surprisingly, a dysfunctional b-RAS is now suggested as a pathological hub in several neuropsychiatric disorders, including anxiety and depressive disorders. ${ }^{235}$

The b-RAS hypothesis may even be able to explain why the cardiovascular situation is so heavily aggravated during stages of PEM. ${ }^{232}$ It has been shown that the brain Ang II/AT ${ }_{1} R$ axis plays a central 
role microglial priming and thus activation of neuroinflammation - one of the possible explanations for the threshold driven transition into PEM after stressful events (for details, see [3.0]).

It is possible that autoimmunity may play an intermediary role in a deranged brain-RAS, e.g., by causing overstimulation of the receptor systems involved. Autoantibodies against the angiotensin II AT1 receptor and angiotensin 1-7 MAS receptor (the latter is activated by Angiotensin1-7 and seems to convey inhibitory effects on inflammation) have recently been found in an investigation of LongCovid patients. ${ }^{236}$ However, doubts have been raised if these autoantibodies may be the single cause of cardiovascular dysfunction. Experimental studies have shown that ischemic or inflammatory cofactors may be needed for AT1-receptor-autoantibodies to exert pathological effects. ${ }^{236,237}$ Here again, the question of a "causal" role of autoimmunity remains open.

\section{The possible role of dysfunctional adrenergic signaling}

In several explanation of the cardiovascular compromise seen in ME/CFS, abnormal signaling via GPC receptors (notably, beta-2-receptors) has been implicated. ${ }^{2,238}$ Indeed, there is evidence from gene expression studies for the existence of a baseline (pre-exercise) adrenergic dysbalance in ME/CFS, with decreased beta- 2 and increased alpha-2A receptor gene expressions (which may be even more pronounced after exercise). ${ }^{154}$ It is unknown if and how the adrenergic receptor dysfunction may relate to a dysfunctional b-RAS (as described above). Another open question is if the adrenergic receptor dysfunction may be causally related to the adrenergic GPCR autoantibodies found in varying proportions of ME/CFS cohorts or if the receptor dysfunction may be a mere consequence of other upstream pathological processes (chronic adrenergic hyperstimulation for instance has also been shown to go along with conformational changes in receptor structures and sometimes even with elevated GPCR autoantibodies). ${ }^{239}$

Another contentious issue may relate to the target question: which tissues or structures may be affected by the (abnormal) adrenergic signaling? Are these tissues located in the brain or in the periphery, or both? Here, some research evidence has accumulated and may be worth reviewing:

- Clearly, adrenergic receptors play an important role in blood vessel regulation, so beta-2dysregulation may result in ubiquitous endothelial dysfunction. However, it needs to be pointed out that as of yet it is not clear which role endothelial dysfunction plays in ME/CFS. Both small and large artery endothelial dysfunction has been demonstrated in ME/CFS patients. ${ }^{240}$ Yet, it remains to be seen if this is a universal feature of ME/CFS. In one study peripheral endothelial dysfunction has been observed in about half of ME/CFS patients (however, by means of a methodology that has also identified endothelial dysfunction in $20 \%$ of normal controls). ${ }^{238}$ So, while endothelial dysfunction appears plausible as a common pathogenetic pathway in ME/CFS it remains to be seen if this is a reliable and ubiquitous finding.

- Also, it may be worth remembering that the majority of adrenergic receptors may be expressed in the brain (especially in the brain regions involved in autonomic activity and regulation). ${ }^{177}{ }^{190}$ As a matter of fact, (nor)epinephrine receptors of different affinity (including beta-2 receptors) are ubiquitous in the brain and may, through differential activation, promote the different arousal states of the mammalian brain. ${ }^{241}$ Adrenergic dysfunction may therefore mediate a host of central pathological phenomena, including 
disordered cognition and memory. ${ }^{112,115}$ Indeed, the cognitive defects seen in patients with MS or Parkinson's and und Alzheimer's disease are now being understood as a consequence of $\beta$-2 receptor dysregulation in astrocytes. ${ }^{55}$

- Another central effect of disordered adrenergic signaling may be an abnormal response to stress. $\beta 2$-adrenergic receptors are widely expressed on glial and endothelial cells where they transmit the effects of norepinephrine release. ${ }^{178}$ Dysfunctional adrenoceptors may therefore be responsible for an exaggerated stress response.

- $\quad \beta 2$-adrenergic receptors are also expressed on immune cells, and there is good evidence for their role in immunomodulation including fostering immune tolerance, ${ }^{179}$ controlling inflammatory input through the vagus nerve ${ }^{180}$ but also affecting neuroinflammation. ${ }^{181,182}$ As a matter of fact, it has been shown that sustained stimulation of $\beta_{2}$-adrenergic receptors can lead to persistent neuroinflammation. ${ }^{183}$ Also, it has been shown that the central regulatory effects on the peripheral cardiovascular system may be modulated by the beta-adrenergic system, including beta-2 effects on vagal function (for details, see [4.10]).

So in summary, it is plausible that adrenergic dysfunction may be part of the pathological matrix in the unique cardiovascular situation of ME/CFS. The same can be said with regards to the brain-RAS. Indeed, both pathological venues may be plausibly implicated in the complex cardiovascular dysfunction seen in ME/CFS, and they may possibly interact. However, it appears plausible in any case that the neuroglial compartment may be heavily involved as a pathological basis.

\subsection{The inflammatory - and possibly autoimmune - nature of ME/CFS}

Clearly, ME/CFS can be described as an inflammatory disease. The majority of ME/CFS cases can be traced back to infectious events of both viral and bacterial origin in epidemiological and case studies. Also, ME/CFS patients uniformly report clinical signs and symptoms of immune stimulation like flue like symptoms, swollen lymph nodes and mucosal inflammation. In a significant subset of ME/CFS patients reactivation of endogenous herpesviruses has been observed or suggested, ${ }^{10,191,242-244245}$ which may be causal phenomenon or a secondary reflection of immune dysregulation. In some $\mathrm{ME} / \mathrm{CFS}$ cases, a genetic background points to susceptibility to autoimmune dysregulation (for details, see [4.4]). Also, autoimmune processes like autoantibodies to GPC receptors have been identified in a significant subset of ME/CFS patients, and some of their targets are also involved in immune regulation (for details, see below). And finally, the only therapies of ME/CFS based on higher level evidence so far have all been immune-related, e.g., the administration of anti-staphylococcal vaccine ${ }^{246,247}$ or Rintatolimod. ${ }^{248,249}$ Other corroborative evidence for the role of immune processes in ME/CFS comes from - albeit uncontrolled - trials of immunomodulatory therapies including IgG depletion therapies and ${ }^{250}$ administration of IVIG (albeit with controversial results).

While the cause of the inflammatory stimulation remains unknown and may vary even within the post-infectious subset (see below), the phenomenological "immunoscape" seems clearer. Here, research has identified altered cytokine networks in ME/CFS, ${ }^{251}$ antigen driven clonal B cell expansion, ${ }^{252}$ TLR-4 activation and NK-cell dysfunction, ${ }^{253,254}$ signs of immune activation after exercise (see [3.0]) and evidence of neuroinflammation (see [4.1]). Indeed, the most conspicuous 
finding in ME/CFS may be the co-existence of central (neuroinflammation) and peripheral inflammation.

A recent investigation of peripheral inflammatory markers in ME/CFS patients may be a case in point. ${ }^{255}$ Among the cytokines which correlated with disease severity, three were general markers of inflammatory activation (IL 7, TNF- $\alpha$ and CCL11). The other three cytokines discriminatory of ME/CFS in this study are indicative of a neuroinflammatory process: TGF- $\beta 1, \beta-N G F$ and CXCL10 (TGF- $\beta 1$ promotes the proliferation of microglia and is involved in determining neuroinflammatory phenotypes; ${ }^{256,257} \beta$-NGF also regulates microglia and is activated in inflammatory insults to microglia; ${ }^{258,259}$ and CXCL10 mediates leukocyte influx across the BBB in a variety of inflammatory CNS diseases. ${ }^{163}$ )

On the clinical plane, in ME/CFS, inflammatory processes and CNS dysfunction are intricately linked. Signs of peripheral immune stimulation go hand in hand with central fatigue and dysfunctional cognitive, sensory or emotional processing. Also, peripheral inflammatory processes are apparently exacerbated after stress overload, as evidenced by clinical signs of immune stimulation during phases of PEM, including flue like symptoms, swollen lymph nodes, sore throat or follicular changes in the mucous membranes.

Unfortunately, the exact cause of the immune stimulation observed in ME/CFS remains unknown. The inflammatory situation could be a reflection of processes like neuroinflammation (see [4.1]), residual infection, reactivation of previous infections (including herpesviruses, see above), persistence of microbial antigens ("viral ghosts"), autoimmunity, or it may even reflect autonomous dysregulation as both adrenergic overstimulation and vagal dysfunction have been shown to be able to affect the immune tolerance and inflammatory reactivity (see [4.4]). Of note, none of these processes are mutually exclusive. Also, it needs to be remembered that CNS neuroinflammatory processes and peripheral inflammatory processes feed into each other, with peripheral inflammation able to activate neuroglia and thus affect many brain functions.

At least some of these explanations may include the role of the central innate immune system and especially the neuroglial compartment. This clearly holds true for neuroinflammation but also for autoimmunity (see below). The coexistence of central and peripheral inflammation, too, may be explained via the astroglial compartment (for details, see [4.10]). Key areas of communication include the neurovascular unit (endothelium), the brainstem and circumventricular organs of the brain, with the vagal nerve playing an important role. ${ }^{184}$ All these components are being modulated or even regulated by neuroglia, esp. astrocytes.

\section{The role of autoimmunity}

There is little doubt that ME/CFS is an "inflammatory" and thus immune-related disorder (see [4.4]). But is ME/CFS an AUTO-immune disorder?

Support for the autoimmune hypothesis comes from several angles. For one, it has been shown that several infectious diseases go along with broadly targeted autoreactivity, which may persist and create a hotbed for the development of autoimmune diseases or reactions (now again confirmed with Covid 19, see [8]). ${ }^{260}$ Indeed, it has been shown that both inflammation and ischemia may create an immune environment in which GPC receptor autoantibodies may develop, possibly establishing a 
vicious cycle in which receptor dysfunction sustains ischemia (or turn ischemia into additional inflammation). Genetic predisposition may also play a role in the inception of autoimmunity, and indeed, in the postviral subset of ME/CFS patients, genetic markers of susceptibility for autoimmune dysregulation have been identified. ${ }^{261}$

The autoimmune hypothesis has recently been strengthened by experimental work on Fibromyalgia which has identified a component of $\lg G$ as the symptom inducing factor. ${ }^{262} \mathrm{It}$ is plausible that the component in question may be an autoantibody (so far not identified in a very broad survey of candidate autoantibodies). At any rate, the factor apparently binds mainly to satellite glial cells in the dorsal root ganglia (a pathomechanism which is commensurate with the clinical manifestations of the syndrome). This new finding from fibromyalgia research clearly raises an intriguing question: If the "sister disease" of ME/CFS is now possibly explained as an (auto)immune process against GFAP positive cells in the spinal cord ganglia - could ME/CFS possibly be explained as a similar process against GFAP positive cells in the brain (mostly astrocytes and microglia)?

In several studies autoantibodies against GPC receptors have been found at increased levels in a subset of ME/CFS patient. ${ }^{2,263}$ While this may be indicative of pathology, such autoantibodies may also be part of the physiologic regulatory repertoire of humans, raising the question if the elevated levels of GPCR autoantibodies found in ME/CFS may be just reflections of an activated tolerance process or part of a preexisting immune signature conferring susceptibility to the development of $\mathrm{ME} / \mathrm{CFS}$ in some subjects. Also, it has been shown that autoantibodies may be created in response to endogenous or reactivated pathogens, or, under certain inflammatory conditions, even to the normal, commensal human microbiome (which may extend to tissues formerly considered sterile). ${ }^{140}$

Elevations of GPCR autoantibodies has been reported for about a third of ME/CFS patients, but this proportion may also be dependent on the assays used. In some studies, these autoantibodies correlate with disease severity or with clinical markers of ME/CFS like fatigue, while in others there is no such correlation. ${ }^{264,265} 263$ Similar GPCR autoantibodies are found in many autoimmune disorders (including POTS, dysautonomia and Complex Regional Pain Syndrome) ${ }^{187,188}$ but also in cardiovascular disorders. Recent experiments in PASC patients with ME/CFS like symptoms have shown that scavenging of GPCR autoantibodies with the aptamere BC007 may result in brisk, persistent improvement of symptoms. ${ }^{266}$ This - if replicated in controlled studies - could support a central pathogenetic role of GPCR-aabs at least in PASC.

There are several theoretical explanations how autoimmunity may fit into the ME/CFS picture:

- For one, as already alluded to, autoimmunity may be a "bystander" or marker of other inflammatory processes. Specifically, in many disorders, neuroinflammation has been associated with a host of autoimmune reactions against all sorts of components of the brain circuitry (the propensity of neuroinflammation to activate autoimmunity or even autoaggression may be related to a breach in the blood-brain-barrier associated with neuroinflammation).

- Indeed, all of the aabs correlated with ME/CFS so far have been shown to possibly correspond to antigens with functional significance in the CNS. This is true, for instance, for adrenergic receptor aabs which are nowhere more densely packed than in the CNS but also for the aabs against endothelin-1- and angiotension-1- receptors recently identified by Freitag et al. ${ }^{263}$ The latter two aabs apparently correlate well with both "central" clinical manifestations of ME/CFS like fatigue and cognitive functioning but also with disease 
severity, global disability and exertion induced symptoms. Also, unlike adrenergic and muscarinergic aabs, these aabs may correlate with disease severity also in the subset of $\mathrm{ME} / \mathrm{CFS}$ patients with gradual onset. Interestingly, both receptors may be intricately linked to neuroinflammation and brain perfusion. The AT-1-receptor has been shown to be a functional hub in the brain-RAS, which plays a role in the regulation of brain perfusion and inflammation (for details, see [4.2]). Endothelin-1-B-receptors are highly expressed in astrocytes, and endothelin 1 (ET-1) has been shown to regulate astrocytic activation, proliferationen and to orchestrate reactive gliosis. ${ }^{267}$ ET-1 production in the brain is accordingly increased during many neurological conditions with an inflammatory response, such as ischemic stroke, TBI, MS, and viral infections. Increased ET-1 levels in the brain have been correlated with exacerbation of neuroinflammation, perfusion defects and ischemic injury (for a review on the role of ET-1 in the CNS, see ${ }^{268}$ ). It is possible that the aab elevations seen in ME/CFS may reflect an inflammatory milieu in these functional circuits, i.e. they could be a marker of neuroinflammation (similar to the anti-GFAP-aabs found in Gulf War Illness) ${ }^{198}$

- Autoimmunity may also play a causal role in CNS pathophysiology. Autoimmune changes in the signaling cascades of the beta-2, the b-RAS system, the endothelin signaling system or the chemo- or baroreceptor system, for example, may plausibly contribute to cerebral blood flow dysregulation and/or neuroinflammation (for details, see [4.1]). The adrenergic receptor population, together with a large variety of other GPCRs (including PGE receptors, chemokine receptors, CCR5 or purinergic receptors - which all may potentially also be affected from autoimmune dysfunction after Covid-19), also have profound regulatory influence on immune processes both in the innate and the adaptive immune system. ${ }^{269,270}$ In fact, dysregulation of $\beta_{2}-A R$ in microglia and in astrocytes, may contribute to neuroinflammation.

- Autoimmunity may also play a role in carrying the disease process to the periphery (e.g., through beta-2-dysfunction - causing skin hypoperfusion, muscular hypoperfusion, orthostatic intolerance etc.) or by inducing vagal dysfunction.

- Autoimmunity may contribute to secondary pathology of clinical relevance like small fiber neuropathy (SFN), assumed to be present in at least one third of ME/CFS patients ${ }^{271}$ (the fact however that SFN - associated with fatigue and postural orthostatic tachycardia syndrome in other disorders ${ }^{272}$ - seems to occur independent of disease severity in ME/CFS may indicate that SFN may not play a primary role in the ME/CFS disease process). ${ }^{273}$

- It has been proposed that autoimmunity in ME/CFS may also affect neuronal/CNS targets other than the GPC receptors. Autoreactivity to a limited number of neuronal proteins including some CNS antigens (including the NMDA receptor) has been tested with no specific autoimmune reactivity found in ME/CFS patients. ${ }^{274}$ (Unfortunately, as of now, antigens related to astroglial activity have not yet been examined for possible autoreactivity, except for in Gulf War Illness, where anti-GFAP has been consistently positive). ${ }^{198}$ Alternatively, targets involved in mitochodrial energy production and/or hormone receptors and ion channel proteins have been proposed as possible hotbeds for autoreactivity: yet, as of now, this is not corroborated by evidence.

So, at this point there are many good reasons to assume that autoimmune phenomena are present in $\mathrm{ME} / \mathrm{CFS}$, yet their contribution to the pathological process, let alone the exact mechanism, remains unclear. 
Some authors have pointed to the clinical similarities of ME/CFS to other autoimmune diseases triggered by molecular mimicry and subsequent dysfunction of a specific enzyme, like Multiple Sclerosis, Primary Biliary Cirrhosis or Rheumatoid Arthritis. ${ }^{261}$ However, these diseases are fundamentally dissimilar to ME/CFS in an important dimension: they all go along with clear-cut tissue damage, scarring and disease progression. To me, a specific, ubiquitous target important for cellular survival does not appear plausible in a fundamentally "functional" disorder like ME/CFS. If an autoimmune target is to be identified in ME/CFS it may rather relate to signal transduction or other functional or regulatory tasks.

Also, it remains to be established if autoimmunity in ME/CFS represents an obligatory process in all of the disparate "etiotypes" (see introduction) or if autoimmunity may be of pathogenetic relevance only in a subset of cases (e.g., the post-infectious etiotype). The notion of a primary autoimmune reaction at the core and beginning of ME/CFS may indeed not go well with the assumption of a common pathological hub for all ME/CFS etiotypes (it has to be acknowledged however, that not much is known about autoimmunity especially in the "orthopedic/connective tissue" category).

Summary: It may be premature to speculate on potential links of autoimmunity to the astroglial compartment. Yet it needs to be recognized that both astrocytes and microglia contribute to CNS autoimmunity by regulating autoantigen-presentation, BBB and thus autoantibody leakage, as well as by activating adaptive $T$ - or $B$ - cell autoantibody production. ${ }^{275-277}$

\subsection{The role of mast cells}

As of now, the role of mast cells in the pathophysiology of ME/CFS is not well understood. There may be a fair number of "allergic" colors in the clinical ME/CFS picture - the conjunctival injection and stuffy nose, the sinus congestion with mucus retention and also sneezing fits occurring at times during exacerbations may (or may not) be related to mast cell dysfunction. Some researchers and ME/CFS practitioners have suggested that mast cell activation syndrome (MCAS) may be associated with ME/CFS in a subset of patients ${ }^{278}$ - unfortunately a contentious issue because MCAS is an illdefined entity with symptoms that partly overlap with ME/CFS. ${ }^{279}$

However, the role of mast cells seems to receive increasing interest both in ME/CFS and in PASC research. Indeed, mast cells are not only "allergy cells" but are recognized as important early effectors of the innate immune response. They reside on the brain side of the BBB (and here, especially in the hypothalamus), ${ }^{280}$ and heavily interact with astrocytes, microglia, and blood vessels. ${ }^{281,282}$ Via their stored and newly synthesized neuroactive mediators (including IL6 and IL-1 beta), mast cell can promote microglia and astrocyte activation and may therefore play a role in neuroinflammation. ${ }^{66,283,284}$ Indeed, it seems plausible from the research record that mast cells may be deeply engaged in the immune functional matrix of neuroinflammation and may actually be "partners in crime" of astrocytes and microglia. ${ }^{66}$ Notably, mast cells can be activated by CRF, a process that has been shown to be induced also by psychological stress ${ }^{285}$ and to be associated with increased vascular permeability and also disruption of the BBB. ${ }^{286}$ This may not only lead to the persistence and aggravation of neuroinflammation but also constitute a link to intracranial hypertension (for details, see [4.9]). 
Interestingly, it has been shown that cerebral mast cells and astrocytes may even cooperate in the genesis of cognitive dysfunction - at least it has been shown that postoperative dysfunction may be caused by mast cells which promote astrocyte activation. ${ }^{283}$ Also, the activation threshold of mast cells seems to be reduced by acute stress which thus adds to a pro-inflammatory tissue milieu and also an increased permeability of the blood-brain-barrier. ${ }^{287,288}$

It is known that mast cells degranulate in response to sheer strain, tissue torsion and stretch, ${ }^{66,289}$ which may explain the frequent association of MCAS and hypermobility syndromes and may also assign a central role to mast cells in the pathophysiological matrix of the neuro-orthopedic and hypermobile cases of ME/CFS (for details, see [5.0]).

Mast cells have been suggested as possible treatment targets for ME/CFS ${ }^{290}$ as well in POTS ${ }^{291}$ and hypermobility syndromes. ${ }^{292}$ The latter two entities are both common co-morbid conditions of $\mathrm{ME} / \mathrm{CFS}$ in which MCAS is frequently suspected as a possible clinical or pathological association (the link between the rare disorders POTS, Ehlers Danlos Syndrome, and MCAS may have a genetic component)..$^{293}$

It is unclear what role mast cell directed therapies may have in ME/CFS but is has been noted that some of the commonly used drugs like Low Dose Naltrexone may also have mast cell stabilizing effects. ${ }^{294}$ Recently, antihistamines have been used as treatment for Long Covid, with surprising albeit preliminary - success. ${ }^{295}$

So clearly, much about the mast cell story in ME/CFS seems to be unwritten, some of the text may indeed depend on a better understanding of how glia interacts with mast cells.

\subsection{The "metabolic trap" hypothesis}

Anyone familiar with ME/CFS research will know the "metabolic trap hypothesis" advanced by Alex Kashi, Robert Phair and Ron Davis on the basis of a big data study of the ME/CFS patients in the public genome databases. ${ }^{296}$ This search identified one gene in which damaging mutations were found in ALL of the ME/CFS patients registered: IDO2, which is one of the two "IDO" genes that encode for enzymes that transform the essential amino acid tryptophan (a precursor for important molecules such as serotonin and melatonin) into kynurenine (the enzymes are called IDO1 and IDO2). The metabolites of the kynurenine pathway (the kynurenines) are important regulators of the immune system and inflammatory responses in general. Both IDO enzymes therefore can affect the immune system because they both produce kynurenin. However, there are important differences between how IDO1 and IDO2 work: when tryptophan is at high levels in a cell, the IDO2 enzyme increases its production of kynurenine while the IDO1 enzyme decreases its production of kynurenine in response to low tryptophan levels. So, if you now have a mutated IDO2 gene (and thus a dysfunctional IDO2 enzyme) the whole IDO-system gets out of whack: the cells must now rely solely on IDO1 to produce kynurenine from tryptophan. Under certain circumstances this situation creates a stalemate: If for any reason the tryptophan levels in a cell rise too high, then IDO1 will stop making kynurenine and tryptophan levels will remain high. This is what Kashi, Phair and Davis call the IDO metabolic trap. 
So far no one knows what (or which constellation of influences) may trigger a "metabolic trap" in $\mathrm{ME} / \mathrm{CFS}$. However, there are two very interesting apects about the tryptophan/kynurenine pathway (which may be relevant independent of the "trap"-hypothesis):

- A well balanced kynurenine pathway seems to be essential for brain homeostasis as one of the metabolites, quinolinic acid, is regarded as neurotoxic, whereas another one, kynurenic acid, is considered neuroprotective (both metabolites also differently interact with the glutamate transmitter system). Therefore it is thought that the normal activation of the kynurenine pathway in IDO competent cells (like microglia and astrocytes) both reduces inflammation and promotes immune tolerance by inducing the proliferation of regulatory $\mathrm{T}$ cells. ${ }^{297}$ Overstimulation or dysfunctional IDO, in contrast, will lead to increased production of quinolinic acid which may foster neuroinflammation. ${ }^{298}$ For example, in schizophrenia, the dysregulation of kynurenine metabolism has been shown to be related to proinflammatory cytokines, attention, and prefrontal cortex volume. ${ }^{299-301}$ Similar findings have been identified in depression. ${ }^{302-304}$ Other studies have related central fatigue as well as memory problems to activation of the kynurenine pathway, again possibly through neuroinflammatory effects of the kynurenine metabolites. ${ }^{305-307}$ This may also explain why, in studies of neuroinflammatory disorders, abnormalities in the tryptophan-kynureninemetabolism correlate with GFAP, a marker of astrocyte activation. ${ }^{299}$ So it appears that the neuroinflammation seen in many CNS disorders may not simply be induced via the "classical" inflammatory pathways but constitute some sort of low grade inflammation possibly initiated and maintained via the Tryptophan-Kynurenine-Pathway. ${ }^{141} \mathrm{~A}$ similar link has been hypothesized for ME/CFS, ${ }^{308}$ and this may be supported by the finding of highly elevated neopterin levels in ME/CFS patients (neopterin levels seem to correlate with the kynurenine metabolism). ${ }^{309}$ Of note, dysfunctional tryptophan-kynurenine-pathways may not only foster neuroinflammation but also directly affect neuronal function, because metabolites of kynurenine also interfere with glutamate and dopamine availability at the synaptic cleft. ${ }^{310}$ The initimate interaction of the kynurenine pathway with the innate immune system and its role in the transmission of peripheral inflammation and CNS inflammation may also explain why a dysfunctional activation of the kynurenine pathway is among the hypotheses to explain the inception of post-infectious ME/CFS (for details, see [7.0]).

- Interestingly, while the classic inflammatory pathways are mediated by IDO enzymes in the microglia, stress-induced inflammation depends on IDO activation in astrocytes only (indeed, of eight Ido2 transcripts, only one - Ido2-v3 - is increased by stress, and it is only present within astrocytes). This seems to indicate that in the stress response the astroglial compartment may play a special role because only this compartment is then activated through the tryptophan-kynurenine-pathway. ${ }^{311}$

So, taken together, there is some evidence that links the "metabolic trap" postulated for ME/CFS to the astroglial compartment, which may indeed be exquisitely susceptible to derangements in the tryptophan-kynurenine metabolism and may translate these derangements into uncontrolled neuroinflammation.

Interestingly, disturbed tryptophan-kynurenine-metabolism has also been observed in COVID-19. ${ }^{312}$ It has therefore been postulated that this may be a potential starting point for the development of long-term symptoms like fatigue, depression and sleep problems (for details, also see [7.0]). ${ }^{297}$ Indeed, it has been shown that the kynurenine/tryptophan ratio remained elevated in some post- 
acute Covid 19 patients and the question was raised if this may be a marker for the development of PASC. $^{313}$

Summary: plausibly, the "metabolic trap" hypothesis may support a central role of the astroglial compartment in the pathophysiology of ME/CFS.

\subsection{Mitochondrial, cellular and "metabolic" dysfunction}

One of the undisputed pathological features in ME/CFS is mitochondrial dysfunction, sometimes explained as an isolated dysfunction of certain mitochondrial processes or explained in the context of a general cellular dysfunction. ${ }^{314}$

As of now, however, it is unclear if the mitochondriopathy seen in ME/CFS is of a specific kind or origin and how this feature may tie into the overall pathological matrix of the disease. After all, mitochondrial dysfunction is seen in a wide range of diseases including autoimmune disorders like MS, in neurodegenerative diseases as well as in cardiovascular disorders. Indeed, in the absence of evidence for a primary mitochondrial defect, the mitochondrial involvement observed in ME/CFS may reflect several upstream pathophysiological processes, especially inflammatory stimulation (from whatever source), generally restricted perfusion ("misery perfusion") or, more specifically, beta-2-dysfunction (the latter has been shown to contribute to mitochondrial dysfunction). ${ }^{314,315}$ This model of explanation is also supported by a recent metabolomics study which shows that the metabolic derangements seen in ME/CFS may not be specific of ME/CFS but rather reflect energy strain caused by hypoxia in exertion-sensitive tissues. Indeed, the two most distinct metabotypes identified in this ME/CFS cohort „,aligned well with known phenotypes of chronic diseases with immuno-metabolic projections". ${ }^{316}$

There is a lot of support for the notion that inflammatory stimulation may go along with or even induce mitochondrial dysfunction. Mitochondrial dysfunction and inflammatory processes have been shown to be closely interlinked. Inflammatory activity, via proinflammatory cytokines can cause mitochondrial fragmentation and dysfunction ${ }^{317}$, and there is now good evidence that inflammation is intimately linked to energy metabolism. ${ }^{318}$ Also, cytokines like TNF alpha can derange mitochondrial energy production and induce mitochondria to produce ROS thereby amplifying the inflammatory process. So clearly, mitochondrial dysfunction may be both cause and effect of inflammatory processes: inflammation may impede mitochondrial function, but mitochondrial dysfunction may also promote inflammation. ${ }^{319}$ Indeed, it has been shown that mitochondria directly respond to DAMPS and are able to rapidly activate the NLRP3 inflammasome, making them a central hub of the innate immune response - which is clearly activated in ME/CFS. ${ }^{320}$ It has even been suggested that viral and bacterial infections or reactivations may directly target mitochondria as part of an evolutionarily preserved adaptive process as the mitochondria are organelles with prokaryotic origin (such such mitochondrial "hijacking" by viral RNA seems to play a role in Zika, Ebola and Influenza A infections, and similar processes have been observed in ME/CFS, where herpesviral reactivation seems to have a profound influence on mitochondrial function). ${ }^{10,321}$

With regards to the inflammation-mitochondria connection, mitochondrial dysfunction may therefore also tie into the neuroinflammatory picture seen in ME/CFS. ${ }^{319,322}$ Indeed, it has been 
shown that inflammatory mediators (including glutamate) produced by activated microglia can trigger intracellular signaling cascades that can alter mitochondrial metabolism including respiratory chain enzyme activity (this may explain the observation of increased lactate levels in the CSF of $\mathrm{ME} / \mathrm{CFS}$ patients). The latter effect on energy production has been observed in MS (where mitochondrial dysfunction also appears to be a part of the pathologic matrix much like in ME/CFS), and clearly shown to be secondary to inflammatory ROS and thus reversible. ${ }^{320}$

Mitochondrial dysfunction could also help explain the clinical cascade that follows exercise induced autonomous dysfunction (PEM). Here, both the inflammatory stimulation and/or the perfusional compromise may rapidly induce mitochondrial dysfunction, which in turn leads to deficient glycolysis with subsequent lactic acidosis, (further) reduced tissue perfusion, increasing production of ROS and release of inflammatory cytokines - all of which may contribute to the clinical manifestations seen in PEM, like psychomotor slowing and central and peripheral fatigue. ${ }^{320,323}$

Given the available evidence it appears plausible that ME/CFS-related mitochondriopathy and the hypometabolic state of this disease in general may be a non-specific part of the disease or a consequence of other pathological processes in ME/CFS. This does not imply that there may not be specific signatures of mitochondrial dysfunction in ME/CFS nor does this mean that it may not be worthwhile pursuing therapies to improve mitochondrial function in ME/CFS. However, pathophysiologically, mitochondriopathy may be but one of the many non-specific "downstream" or "mirror" phenomena of ME/CFS. ${ }^{324}$

Summary: mitochondrial dysfunction seems to be ubiquitous in diseases marked by inflammation and/or reduced blood flow and may indeed be a final pathophysiological pathway in a wide range of tissues. As of yet there is no evidence of a primary pathophysiological role of mitochondrial dysfunction in ME/CFS.

\subsection{Abnormal cerebral blood flow}

The human brain is the recipient of 20 to $25 \%$ of our total blood flow and its functions heavily depend on an exact distribution and regulation of this blood flow.

One of the most consistent (if not THE most consistent) finding in ME/CFS research is an abnormal brain perfusion. ${ }^{93,95,325-331}$ With the advent of novel dynamic imaging studies it could be shown that practically ALL ME/CFS patients suffer from brain hypoperfusion in response to autoregulatory challenges including head tilt maneuvers and cognitive exercise. ${ }^{332,333}$ In both the head tilt studies and in other studies using indirect markers of brain perfusion, the degree of hypoperfusion correlates well with symptoms and clinical severity of ME/CFS. ${ }^{5}$ Indeed, the finding of impaired cerebral perfusion appears so central in ME/CFS (and now in PASC) that it may be worthwhile to examine any remedial intervention suggested for ME/CFS for its effects on cerebral perfusion.

Interestingly, in an experimental study it has been shown that cerebral blood flow in ME/CFS patients can be increased by the administration of the alpha 1 agonist phenylephrine and that symptoms like 
brain fog and cognitive dysfunction subside after the injection. ${ }^{334}$ Because phenylephrine does not cross the BBB the authors suspect that this may be an effect of a higher perfusion pressure which may overcome the hypoperfusion bottleneck or that epinephrine may alter the baroreceptor setpoint.

As of now, the cause of the blood flow problems in ME/CFS is not known. It has been hypothesized that this dysregulation of cerebral blood flow may be related to inappropriate vasodilation as a result of neuroinflammation and/or that it may be related to abnormalities in the function of the chemoand/or baroreceptor circuits with a subsequent mismatch of ventilation, blood pressure and perfusion (for details, see below). Perfusional defects may also result from "mechanical" factors like reduced brainstem mobility (as seen in tethered cord syndrome), repetitive strain injuries (as possibly related to $\mathrm{CCl} / \mathrm{AAI}$ ) or cerebral hypertension (as seen in many ME/CFS patients with hypermobile Ehlers Danlos syndrome) (for details on these "hypermobile and/or "neuro-orthopedic" cases, see [5.0]). Interestingly, it has been shown that ME/CFS patients wit joint hypermobility syndromes have larger cerebral blood flow reductions during tilt table test than ME/CFS patients without hypermobility. ${ }^{335}$ Of course, cerebral perfusion defects may also be a consequence of "upstream" regulatory defects, i.e. from defects or dysfunctions in other brain regions, if related to neuroinflammation, disturbed signaling (e.g. in the framework of autoimmune processes), vagal dysfunction or generally to an increased sympathetic tone.

Regulatory brain regions involved in blood flow regulation may include the brain-RAS, ${ }^{336}$ the reticular activation system ${ }^{5}$ and the circuits involved in the functions of the carotid body chemoreflex and the baroreflex (see below). ${ }^{334}$

How may the findings of a dysfunctional cerebral blood flow regulation in ME/CFS patients relate to the neuroglial compartment? Astrocyte and microglial activity has been shown to influence cerebrovascular autoregulation ${ }^{95,337}$ and cerebral blood flow. . $7,42,338$ Indeed, cerebrovascular autoregulation is a function of the arterioles which control blood flow into the capillaries, and here astrocytes seem to play a central role because both the baro- and the chemosensory circuits responsible for instantaneous adaptation of blood vessel function and blood flow are heavily dependent on astrocyte function. ${ }^{26}$ Also, it has recently been shown that neurovascular structure and function of the CNS capillaries is regulated by P2RY12 receptors on capillary-associated microglia. ${ }^{27}$ Of course, cerebral blood flow is also regulated by the baroreceptor circuits (for details on possible baroreceptor involvement in ME/CFS, see below).

To make matters more complex, cerebral hypoperfusion may also cause some of the above pathologies. A deficient energy supply for instance may cause neuroinflammation, brain edema, an altered carotid body chemoreflex, adrenergic hyperstimulation or a disrupted BBB with all associated consequences. Yet, in all these connections the astroglial compartment seems to play an important role.

\section{The role of a dysfunctional baroreflex}

There are some suggestions (like decreased blood pressure variability) ${ }^{339}$ that baroreflex problems may be involved in the blood flow dysregulation seen in ME/CFS. Baroreceptors in the large blood vessels control arterial blood pressure beat-to-beat and thus match blood pressure with cardiac output. If the baroreflex is not set adequately (or if the brainstem is not interpreting the signals from 
the baroreceptors correctly) the cardiovascular system will not be able to respond to fluctuating blood flow demands in response to stress (like exercise or positional changes, for instance).

The proper functioning of the baroreflex seems to be of vital importance for exercise tolerance. Indeed, basic cardiovascular research has shown that an accelerated baroreflex in the presence of blood pressure decrements (a plausible combination in ME/CFS) can lead to insufficient exercise tolerance, chronotropic incompetence and sympathetic activation during low-intensity exercise (which again are all features of ME/CFS). ${ }^{340}$ This may possibly link baroreceptor problems to PEM (for more on PEM, see [3.0])

The regulation of the cardiovascular system via the baroreflex is coordinated in the nucleus of solitary tract (NTS, for details, see [4.10]) and is heavily dependent on NTS astrocytes which also respond to vagal and adrenergic input and are thus able to modulate the cardiovascular reflexes. Also, baroreflex sensitivity seems to be influenced via angiotensin II type 1 receptors as well as brainderived neurotropic factor levels in the NTS. ${ }^{230,231,341}$ Baroreflex sensitivity may therefore also be tied to proper functioning of the b-RAS and epinephrine levels (for details on the b-RAS, see [4.2], for epinephtine levels in ME/CFS, see [4.11]). ${ }^{334}$

It has also been shown that neuroinflammatory processes involving the NTS can easily inhibit the baroreflex centrally through ATP release from activated NTS glia. ${ }^{26}$ Indeed, reactive astrogliosis in the NTS has been reported after CNS trauma, infection, ischemia, stroke, and in autoimmune disease. ${ }^{48}$

In summary, there are many indications that the abnormal cerebral blood flow observed in ME/CFS may be related to abnormal astroglial functioning.

\subsection{Intracranial Hypertension}

Given its relative rarity, intracranial hypertension is a surprisingly common finding in ME/CFS. In a large MRI study, $42 \%$ of patients meeting the ME/CFS Canadian Consensus Criteria were found to have MRI signs of intracranial hypertension. ${ }^{342}$ Several years before, borderline raised intracranial pressure (ICP) was found by lumbar puncture in most of the $20 \mathrm{ME} / \mathrm{CFS}$ patients examined, and a pathologically elevated ICP was found in $4 / 20$. Cerebrospinal fluid drainage improved symptoms in $17 / 20$ patients, yet only temporarily. ${ }^{343}$ Interestingly, the brain area most vulnerable to alterations in cerebrospinal fluid pressure appears to be the dorsal brainstem, ${ }^{344}$ i.e., an area heavily implicated in ME/CFS pathology (see [4.9]).

Intracranial hypertension has especially gained recognition in the "hypermobile" cases of ME/CFS as a significant association between intracranial hypertension and Ehlers Danlos Syndrome (EDS) has been noted in several studies, including observations that symptoms improved with symptoms improved with venous stenting. ${ }^{345,346}$ In a large case series of 272 severely affected ME/CFS patients, possible signs of increased ICP were found in $83 \%$; $32 \%$ were classified as definitely pathological. ${ }^{342}$ Notably, hypermobility was identified in $50 \%$ of the cases and a surprising $56 \%$ of all patients had signs of downward displacement of cerebellar tonsils . Additionally, signs of craniocervical obstruction were found in 100/272 patients. 
So clearly, there is a large overrepresentation of hypermobility, signs of intracranial hypertension and also craniocervical obstructions in the more severe ME/CFS cases (for details, see [5.0]). The cause of the association between intracranial hypertension and ME/CFS is unknown. It is also unknown if the high prevalence of possibly raised ICP may be more or less limited to the "hypermobile" and or "neuro-orthopedic" subset of ME/CFS or if - and to what degree - it also extends to the postinfectious cases, for instance.

Possibly, intracranial hypertension could arise from restricted cerebrospinal fluid circulation/reuptake due to mechanical compression. Also, BBB disruptions, blood flow dysregulation, perfusional defects, neuroinflammation and associated brain edema may play a role. Also it has been shown that BBB disruption during neuroinflammation may cause fibrinogen extravasation. ${ }^{347}$ This may compromise cerebrospinal fluid flow and intracranial pressure regulation which relies on functional elasticity of the perivascular spaces. ${ }^{348}$ Notably, there is some evidence of punctate hyperintensities in the brain of ME/CFS patients which may or may not be associated with extravasation of fibrinogen. ${ }^{349}$ (Of note, the integrity of the the BBB is maintained by astrocytes and has been shown to be affected by stress exposure, activated Tryptophan/Kynurenine pathways, systemic inflammation and neuroinflammation as well as an overactived brain-RAS - which all may be of relevance in $\mathrm{ME} / \mathrm{CFS}) .{ }^{350}$

Interestingly, a patient driven initiative has recently suggested an investigation of high dose thiamine as a possible therapeutic agent for ME/CFS after collecting case reports from patients who had tried the medication..$^{351,352}$ It has been suggested by the initiator of this pilot study that high dose thiamine may act in similar ways as acetazolamide, a known diuretic that may decrease intracranial pressure. As of now, it is unknow if and how this therapy holds up in a controlled study and which subset of $\mathrm{ME} / \mathrm{CFS}$ patients may be responders.

In summary, much needs to be learned about the association between intracranial hypertension and ME/CFS and how it ties into other recognized pathological entities like decreased cerebral blood flow or neuroinflammation.

\subsection{Autonomous dysfunction}

Clearly, ME/CFS is marked by a profound dysbalance between the sympathetic and parasympathetic nervous systems, indicative of autonomic dysfunction, many features of which have been separately described in this review (for instance, cardiovascular dysfunction, see [4.2] or vagal dysfunction, below).

Here it may suffice to say that there is no doubt in the literature that GFAP positive glial cells (i.e. astrocytes) are implicated in modulating autonomic functions on many regulatory levels including modulating vagal input from the periphery ${ }^{52}$ and generally regulating the complex autonomous physiology and behaviour. ${ }^{353}$ Hereby, a central role is ascribed to the nucleus of the solitary tract (NTS) and other nuclei of the dorsal brainstem. The NTS, for example, is the primary brainstem center for the integration of physiological information from the periphery and not only contributes to the control of cardiovascular, respiratory, glucoregulatory, and gastrointestinal functions ${ }^{52,190,354}$ but also integrates the vagal input from the periphery and thus contributes to inflammatory 
homeostasis. Indeed, the dorsal brainstem can be considered a central hub both for autonomous neurocircuitry and for the regulation of inflammation. ${ }^{355}$

Summary: the relationship between autonomous function and the functions of the astroglial compartment is as tight as can be; autonomous dysfunction can only be conceptualized from the assumption of astroglial dysfunction.

\section{Vagal dysfunction}

The vagal nerve is actually not a nerve but a system of nerves with over 100000 fibers, $80 \%$ of which transmit signals to the brain, and $20 \%$ send signals from the CNS to the periphery. There is good evidence for vagal dysfunction in ME/CFS, ${ }^{356-358}$ and given the many implications of a competent physiological vagal regulation the effects may be far reaching.

For one, the vagal nerve influences how the signals from the periphery are integrated into CNS functions - a process that happens in the in the nucleus of the solitary tract (NTS) and the dorsal vagal complex of the brainstem. In these "relay stations" of sorts, the vagal input - be it signals from the gastrointestinal system, the lungs or from baroreceptors from the aortic arch - becomes part of the regulatory physiological matrix that modulates cardiovascular function, respiration, and gastrointestinal motility.

The above centers - together with the vagal nerve as a whole and the parabrachial nuclei - also play an important role in the immune system's homeostasis. For one, these centers transmit inflammatory signals from the periphery to the limbic system and the neocortex. Also, when the NTS detects, via the vagus nerve, pro-inflammatory cytokines from the periphery (such as tumor necrosis factor-alpha or interleukin 1-beta), the local glial cells - microglia and astrocytes - are activated. This in turn stimulates the CNS innate immune system and elicits an inflammatory response in the CNS (this body-brain communication is called afferent neuroimmune signaling). Also, during systemic inflammation, stress-regulating brain areas are stimulated in response to vagal activation of the dorsal vagal complex in the brainstem. It is thought that this is the physiological basis of the behavioral sickness response that humans and animals display during infections. ${ }^{50}$

While the vagus nerve is part of the immunological body-brain and brain-body axis, the vagal nerve also plays an important role in modulating inflammation - in fact, it can be considered a major regulator of the immune system. ${ }^{359} \mathrm{~A}$ well-functioning vagal nerve is able to tone down central inflammation, while a dysfunctional vagus nerve results in an overactive immune response, inflammation and possibly autoimmunity (the latter process has been implicated in POTS). ${ }^{360}$ Not surprisingly, the extent of heart rate variability suppression correlates with the levels of systemic inflammation. ${ }^{361,362}$

With regard to the widespread beta-2-dysfunction suggested in ME/CFS it bears noting that the inflammation-modulating effects of the vagal nerve are in part mediated by beta-2-adrenoreceptors, so this is another venue through which beta-2-receptor dysfunction may lead to central pathology. The modulating effect of the vagal nerve on central inflammation has been suggested as a rationale for vagus nerve stimulation as therapeutic venue against neuroinflammation, and first promising therapeutic effects of vagal nerve stimulation have recently been reported from a randomized controlled trial in ME/CFS patients. ${ }^{363}$ 
The vagal centers of the brainstem are also heavily connected with upstream regulatory CNS centers, (e.g., in the hypothalamus) and thus affect the HPA axis as well as the "limbic" functions of the CNS. These multi-level remote effects of vagal dysfunction may explain why one of the most prominent hypotheses of the pathogenesis of ME/CFS starts from the suggestion of a possibly persisting vagal infection. ${ }^{190}$ Vagal dysfunction may however not necessarily be related to vagal infection and may also start from any pathology in the vagal relay stations of the vagal nerve, no matter how it may be caused. $^{344}$

How does all this relate to astroglial functions? For one, astrocytes are an integral part of the regulatory matrix in the NTS and dorsal vagal complex. If they don't function well the gatekeeping role of these centers will be compromised. Also, vagal dysfunction is closely tied to glial activation and thus neuroinflammation.

In summary:the vagal dysfunction observed in ME/CFS may only make sense in consideration of the physiologically pivotal role of astrocytes.

\subsection{The abnormal stress response}

ME/CFS is a "paradoxical" disease as it covers both sides of the arousal spectrum: fatigue, cognitive dysfunction, motor slowing on the one hand - but hypervigilance, overstimulation, insomnia and sensory overload on the other. Indeed, ME/CFS has been described both as state of "hibernation"364 and of "sustained arousal". ${ }^{8}$ This overextension may also explain the frequent admixture in ME/CFS of deep emotional disturbances including anxiety, panic and the feeling of existential threat.

How could this paradoxical combination be explained?

One explanation may be that adrenergic hyperstimulation and vagal dysfunction coexist, the adrenergic system is unchecked (for details on vagal dysfunction in ME/CFS, see [4.10]).

The adrenergic runaway train with its high epinephrine load has been explained as a feature of beta2-receptor dysfunction (see [4.2]). ${ }^{112}$ Alternatively, the high epinephrine levels may reflect a dysfunctional brain Renin-Angiotensin-System (see [4.2]), brainstem hypoperfusion (which has been shown to increase norepinephrine output in the locus coeruleus) or high lactate levels in the brain possibly associated with neuroinflammation (lactate has been shown to be an important neuromodulator which also influences norepinephrine release in the locus coeruleus). ${ }^{365,366}$

Other explanations (which may or may not be mutually exclusive with the former explanations) have tried to tie the adrenergic overstimulation into the wider context of an abnormal stress regulation. Indeed, the "hyperarousal" part of ME/CFS may well be summarized as evidence of a dysfunctional response to stress, i.e., an inability to muster the physiologic allostatic load. This has been explained as a consequence of reduced cerebral blood flow, ${ }^{331}$ of a generally heightened sensitivity of the CNS to all sorts of stressors (be they physical, cognitive or orthostatic), ${ }^{367}$ or of inflammatory processes (be they infectious, neuroinflammatory or autoimmunity related) that may render the stress response system dysfunctional and thus the stress response inadequate. The assumption of a dysfunctional response to stress has also led to the suggestion that it may be the very brain centers and receptor systems involved in the normal stress response that may be dysfunctional in ME/CFS. 
- Here, some attention has been given to the hypothalamus - which can be considered the "stress response center" of the brain as it is targeted by stress signals on any level, be they physical, immunological (e.g., infection related), mental, cognitive or emotional in nature. Here incoming stress signals are received and processed in the paraventricular nucleus (PVN) which then orchestrates the appropriate responses (including those in other brain centers, most prominently the limbic system in which many of the brain centers highlighted as possibly affected in ME/CFS are located, including the amygdala, cingulate cortex and hippocampus). Not surprisingly, a PVN rendered dysfunctional by localized glial activation has been suggested as the substrate of the dysfunctional stress response in ME/CFS. ${ }^{7}$

- Interest has also been given to the neurotransmission via Corticotropin Releasing Factor (also named CRH, for Corticotropin Releasing Hormone), its binding protein (CRFBP) and corresponding receptor systems in the brain (CRFR1 and 2). This "CRF-ergic system" is directly involved in initiation and cessation of the stress response and may therefore plausibly be a central hub in an individual's ability to cope with a stressor. ${ }^{368}$ Its central component, CRF, is expressed in the paraventricular nucleus of the hypothalamus, in the amygdala and in the brainstem - regions that have been implicated in ME/CFS pathology. As a potent neuromodulator which interacts with many transmitter systems in the brain (including serotonin release in the limbic system), CRF has been implicated in many disorders related to exposure to stress and maladaptive stress responses, including neuropsychiatric disorders but also inflammatory diseases. ${ }^{369}$

CRF may also be linked to the modulation of inflammatory responses in the brain. After all, CRF-receptor 1 and 2 are both expressed on microglia and astrocytes and affect the release of cytokines and chemokines. ${ }^{370-372}$ Also, CRF has been shown to have effects on the innate immune system by influencing the nuclear factor kappa B pathway and may thus again, albeit indirectly, play a role in neuroprotection (or neuroinflammation). ${ }^{373,374}$ Studies have shown that chronic activation of CRF receptor 2 promotes an anxiety-like state, yet with attenuated behavioral and hypothalamic-pituitary-adrenal axis responses to stress, clearly reminiscent of the "paradoxical" clinical picture of ME/CFS. ${ }^{375}$ Currently an open trial is testing the hypothesis that downregulation of CRFR2 by the receptor agonist CT38s may improve symptoms in ME/CFS. ${ }^{376}$

- Another hypothesis has focused on the locus coeruleus (LC) as another central component of the physiological stress response system. ${ }^{12}$ The LC is located deep in the brainstem and produces the excitatory catecholamine norepinephrine (noradrenaline) and thus regulates sympathetic nervous system activity. ${ }^{377}$ The LC-system comes to mind in ME/CFS research because its activation is a critical step in mediating the neuroendocrine and behavioral limbs of the stress response ${ }^{378}$ but is also involved in arousal regulation, including regulation of the sleep-wake cycle, facilitation of attention, cognition, mood, memory, blood flow regulation, metabolism, distribution of glucose and oxygen throughout the brain and in the modulation of central inflammatory responses. ${ }^{379}$ The $L C$ noradrenergic fibers reach nearly the entire neuroaxis, including the neocortex ${ }^{380}$ and the hippocampus, amygdala, hypothalamus and thalamus. ${ }^{381,382}$ In turn, the $L C$ receives afferent projections from various brain regions, including the insular cortex, the hypothalamus, the central amygdala, and the cerebral cortex. ${ }^{383}$ Receptors for norepinephrine of different affinity (including beta-2 receptors) are ubiquitous in the brain and may, through differential activation, promote different arousal states. $^{241}$ A very close functional relationship has been documented between the $L C$ and the PVN of 
the hypothalamus; ${ }^{384}$ the latter may directly modulate the activity of LC neurons and may integrate autonomic responses by influencing $L C$ neurons. This relationship may be pivotal for the management of acute and chronic stress. ${ }^{385}$ Also, the LC system is intimately tied to the CRFergic system described above (CRF engages the LC especially during acute and chronic physical and cognitive stress). Of further note, the LC may work in resonance with the salience network (SN), an area including the anterior cingulate cortex that has been implicated in ME/CFS ${ }^{4,386}$ (and now also in Long Covid). ${ }^{387,388}$ So it may be no surprise that the LC, with its far reaching, multi-level involvement in stress and arousal regulation, has been implied in ME/CFS (and other diseases like posttraumatic stress disorder). ${ }^{389}$

Physiologically, both the stress response and the immune response seem to be intricately linked because the stress response is based on intense crosstalk with the transcription factor Nuclear Factor-kappa B (NF-kB), a potent activator of the innate immune response and implied by several groups in the pathology of $\mathrm{ME} / \mathrm{CFS}^{390,391}$ and indeed closely related to fatigue symptomatology. ${ }^{392}$ Inflammatory processes and the stress response are also linked on other functional levels (and, as frequently observed, cerebral blood flow functions may be included in this link). High lactate levels, for example, e.g. as a consequence of hypoperfusion or neuroinflammation, seem to have important neuromodulatory effects as they can set the "homeostatic tone" of the brain, ${ }^{365}$ and thereby closely interact with norepinephrine release in the locus ceruleus. ${ }^{366}$ The three systems are also linked through the adrenergic receptor system as well as the angiotensin II signaling system (b-RAS).

And, of course, they are linked by the functional properties of the astroglial tissue, which always affects vascular regulation, immune regulation and neuronal function in unison.

Indeed, with regards to the above regulatory circuits, it must be postulated that the regulation of the stress response is a complex neuroimmune process that can only be understood in the light of a central role of the astroglial compartment. The latter may also explain the "paradoxical" autonomous dysregulation seen in ME/CFS, in which both the sympathetic as well as the parasympathetic autonomous nervous system are dysfunctional. After all, the astroglial compartment is the central regulatory matrix of both the vagal and the adrenergic autonomous system.

In our opinion, the feature of a "paradoxical" stress response in ME/CFS has even more implications for theory building:

- it undermines simplistic explanations of ME/CFS. No, ME/CFS is NOT a hibernation or "dauer" state - no animal would survive for long with a cardiovascular system revved up like this. And no, ME/CFS is not "sustained arousal" either - after all, as any CFS-patient can testify, ME/CFS-sufferers are clearly under-aroused (and depressed at times).

- This feature may also limit the hopes of fixing CFS by turning off the arousal part (e.g., by administering anti-adrenergic drugs like Clonidine, as tried by Wyller et. al.) ${ }^{393}$ or by fixing the "hibernation" part alone (e.g., by administering mitochondrial boosters, whatever these may be).

- This feature may also advise against the assumption of a single brain nucleus or a single receptor system or a single neurotransmitter system that may initiate the pathological cascades in CFS. These centers and systems may all be involved in the pathology of ME/CFS but they are likely to be part of a wider orchestra (a very important issue that we will revisit in the final discussion, see [7.0]) 


\subsection{Evidence \#5}

\section{The "hypermobility" and "neuro-orthopedic" enigma}

If there is anything surprising about ME/CFS (apart from its "fascinating" clinical picture including features like PEM, but also its "paradoxical" quality) then it may be the fact that many of the patients diagnosed with ME/CFS seem to have additional or underlying issues with connective tissue disorders and/or with neuro-orthopedic disorders.

Up to $50 \%$ of ME/CFS patients may suffer from any of the hypermobility spectrum disorders, including the hypermobile form of EDS (hEDS). ${ }^{394-397}$ Conversely and very conspicuously, the majority of patients with hypermobile forms of EDS meet the criteria for ME/CFS; indeed, ME/CFS seems about 10 times more common in these patients than in the normal population. ${ }^{397}$ Another part of ME/CFS patients has been - related or unrelated to connective tissue disorders - diagnosed with cranio-cervical instability/atlanto-axial instability (CCl/AAI), tethered cord syndrome, Arnold Chiari Malformation or syringomyelia, i.e., with neuro-orthopedic conditions of the spine, base of the skull and adjacent tissues including spinal cord and brainstem. Some of the ME/CFS patients in this category have been completely cured of their "ME/CFS" after correction of their underlying (neuroorthopedic) condition (for a patient report, see $^{398}$ ).

There is some indication that there may be a shared pathological venue in patients with hypermobility problems and those with neuro-orthopedic conditions. Indeed, about one in fifteen patients with hEDS develops CCI/AAl; also, there seems to be a higher prevalence of several of the neuro-orthopedic conditions in hEDS, including Chiari malformations, tethered cord syndrome and syringomelia. ${ }^{345}$

The symptomatology of the "hypermobile" cases with ME/CFS seems to be indistinguishable from other etiologies of ME/CFS and includes fatigue (fatigue is reported in up to $84 \%$ of hEDS patients and it is oftentimes severe and debilitating), ${ }^{399}$ cognitive alterations (memory, concentration), muscle and joint pain; swollen lymph nodes; hypersensitivity to sound and light, non-restorative sleep; and autonomic nervous system dysfunction, including orthostatic intolerance as well as post-exertional malaise. ${ }^{397,400}$

So, what may render patients with hypermobile syndromes and/or one of the neuro-orthopedic conditions listed above susceptible to ME/CFS?.

It has been suggested that connective tissue issues may set the stage for vascular pathology. Yet, at least in hEDS (a well described entity within the hypermobile spectrum disorders), vascular anomalies do not seem to be present. ${ }^{401}$ Another causal factor could be some underlying immune related pathology, and indeed, in hEDS and hypermobile spectrum disorder some possible, albeit ill defined, immune aberrations have been noted, including aberrant transcription of several genes related to inflammation, and immune responses. ${ }^{402}$ Yet, if a vulnerable immune system was to explain the association with ME/CFS one would expect the hEDS/HSD cases to be among the postinfectious cases of ME/CFS, which remains contentious for lack of data. It has been suggested that 
connective tissues may be damaged during the host defense against certain pathogens, ${ }^{403,404}$ which may set genetically vulnerable individuals (e.g. those in the hEDS spectrum category) up for "mechanical" problems induced by infections. As of now, this again remains speculative.

A more plausible lead to a possibly shared pathophysiological basis may start from the common denominator that both the "hypermobile" and the "neuro-orthopedic" cases of ME/CFS may have, namely mechanical strain. In the hypermobile cases collagen is altered in a way that allows tissue to be pulled beyond normal limits. This may interfere with the protective functions of the connective tissues and set the stage for impact injury to adjacent tissues. These strain injuries may not only result in altered joint functions (which may explain the link between hypermobility and CCI/AAI) but also cause dysfunction or even injury in unprotected neuronal tissue, for instance in the spinal cord, the medulla oblongata or the brainstem. Here, two of the nuclei most intensely followed in ME/CFS imaging research, the nucleus of the solitary tract and the dorsal motor complex of the nervus vagus (see [4.10]) could be easily affected from tethering or mechanical compression and related consequences like ischemia, lactate release, hypoperfusion, edema, raised intracranial pressure, or inflammation.

The same "mechanical strain" explanation may apply to the neuro-orthopedic cases, as both Arnold Chiari Malformation, tethered cord syndrome and syringomyelia are marked by traction on the brain stem, which may lead to chronic compression or distortion of neuronal tissue in the brainstem or spinal cord.

But how may the "mechanical" cases fit into the clearly "inflammatory" picture of ME/CFS? I think that these cases go indeed well with the neuroinflammatory theory of ME/CFS if we take a closer look at the pathology involved in these "mechanical" cases :

- For one, the mechanical effects may cause cerebral perfusion problems, e.g., hypoperfusion, but also inadequate blood flow regulation, venous congestion and raised cerebrospinal fluid pressure - with expectable effects on the functions of the neuronal tissues or regulatory centers involved. This "mechanical-perfusional" explanation may fit well with the observation that both intracranial hypertension and "hypermobile" conditions may be found in up to half of the ME/CFS patients. ${ }^{342,343}$ It may also fit with experiments in ME/CFS patients, in which a 15 minute longitudinal neuromuscular strain through unilateral straight leg raise increased symptoms in a subset of patients (it is unknown, however, if this subset correlates with preexisting conditions like hypermobility or intracranial hypertension or if this may be a general response of a "hyperreactive" ME/CFS nervous system). ${ }^{405}$

- Also, the primary "mechanical" injury may easily translate into an inflammatory picture because the deficient energy supply resulting from hypoperfusion and ischemia may activate the inflammatory response and lead to a neuroinflammatory picture. ${ }^{101}$ Indeed, from what we know about neuroinflammation (see [4.1]) it would be surprising if both repetitive strain injuries or injuries marked by compromised brain perfusion not finally ended up in an inflammatory reaction. Mouse models show how easily deformative stretch can cause pathological calcium influx, ${ }^{406}$ altered gene expression, ${ }^{345}$ and apoptosis of neurons, ${ }^{407,408}$ and also how easily neuroinflammation is triggered by repetitive mechanical strain. ${ }^{409}$ The latter link is also clearly borne out by data from traumatic brain injury or repetitive concussions in humans which are always associated with neuroinflammation (and at times can lead to a clinical picture widely overlapping with ME/CFS). ${ }^{410}$ 
- Also, there are some indications that mechanical strain can directly activate inflammatory processes through mast cell activation ${ }^{66,289}$ (for details on the possible connection between mast cell activation and ME/CFS, see [4.11]).

So clearly, the effects of "mechanical" strain can be far reaching.

For me the pathological chain in the "hypermobile" and "neuro-orthopedic" cases most plausibly may look like this: Mechanical strain may lead to cerebral perfusion problems (i.e., hypoperfusion or mismatched perfusion and possibly also venous congestion). The blood flow problems in turn may cause hypoxia and cellular dysfunction leading to the activation of pro-inflammatory cascades which may give rise to neuroinflammation. Blood flow issues may also explain the intracranial hypertension frequently found in these cases (for details, see [4.9]).

Here again the astroglial compartment may play a central role. It has been clearly shown that the neuroinflammation after traumatic brain injury is mediated through astrocytes and microglialastrocyte crosstalk. ${ }^{411}$ It has also been shown that spinal injuries (as to be assumed with tethered cord syndrome or syringomyelia) can induce central neuroinflammation, ${ }^{412}$ and the role of astrocytes in the initiation and maintenance of neuroinflammation is uncontested (see [4.1]). Also, the interplay between mast cell function and astrocyte function is well recognized (see [4.11]).

Now, a point has been made that these "mechanical" cases "are not ME/CFS". This, however, is a weak assertion in a disease that is defined by clinical criteria only. Most "mechanical" cases meet these criteria, so they are ME/CFS, if clearly with a twist.

What else may this special "etiotype" tell us? For one, it supports what can be considered a topographical explanation of ME/CFS: it is in the brain. And: In ME/CFS, some pivotal process must render brainstem perfusion dysfunctional - and this may then well be the overarching pathological feature in all etiotypes of ME/CFS (this pivotal discussion will be covered at the end of this working paper, see [7.0]).

\subsection{Conclusion: A unifying theory of ME/CFS}

The above evidence base may lend itself to several conclusions about ME/CFS:

\section{Conclusion 1}

The underlying pathology must be located at the most basic biological level. From its clinical presentation, ME/CFS can indeed be regarded as one of the most "fundamental" diseases imaginable: ME/CFS not only affects all and any of the "metazoan" homeostatic functions of the human body (like the inborn immune system, inflammation management, arousal and vigilance, sleep, temperature regulation, stress response, locomotion and digestion), but also the "limbic" and 
higher mammalian functions including the reward system, mood regulation, cognition, memory and word processing.

\section{Clearly, the pathological matrix of ME/CFS involves the most basic adaptive level in the human body.}

\section{Conclusion 2}

The pathology of ME/CFS plays on a regulatory level. Within the kingdom of diseases, ME/CFS is a very special disease: It does not resolve. Once fully established it typically does not get worse over time (as degenerative, neoplastic or most autoimmune diseases do). Nor does it follow the pattern of most functional disorders like migraine, which are usually episodic with complete well-being in between. ME/CFS neither resolves, nor progresses nor comes in bouts out of the (healthy) blue. It lingers, with ups and downs of course, but within a clearly abnormal corridor.

For a devastating disease with obvious evidence of chronic inflammation this is remarkable: the defects in ME/CFS seem to remain benign in nature rather than destructive or cytopathic (which is not to say that secondary dysfunctions and even tissue damage may not occur in ME/CFS, yet this seems to happen to a surprisingly limited extent when measured against the severity and depth of the pathology involved). As a matter of fact, if tomorrow a cure for ME/CFS was found, I would expect the vast majority of ME/CFS patients to rise out of their misery like phenix from the ashes.

I see this as evidence that the pathology of ME/CFS plays on a deep regulatory level. Possibly on a regulatory level so profound that I want to raise another question - the question if these circuits are really diseased processes, i.e., truly of a fixed pathological nature. Possibly, ME/CFS is playing on physiologic pathways that are normally only activated for short periods of time and under unusual circumstances (like fighting a disease or other threats) - and that for some reason have become "hijacked" at the onset of ME/CFS and from then on have remained perpetually activated. So rather than representing biology truly gone kaput, ME/CFS may play on a keyboard of evolutionarily preserved, adaptive emergency pathways. ME/CFS, in this sense, could therefore represent a pathological fixation of physiologic "preparedness" responses - i.e., a maladaptation.

This explanation of a "perpetuated" physiologic rescue response may be somewhat reminiscent of the "metabolic trap hypothesis" as advanced by Robert Phair and Ron Davis (for details, see [4.6]), which describes ME/CFS as an entity which is neither a disease nor a biological breakdown of any sort but an epigenetic fixation of a highly unusual biological situation ("the trap model does not suggest a failure of healing or, indeed, a failure of anything at all"). ${ }^{296}$

\section{Conclusion 3}

The profound dysregulation underlying ME/CFS is seated in the central nervous system. Anyone who has worked through this working paper may understand why this is the only plausible assumption for me: ALL the phenomena of ME/CFS can be most directly explained as a consequence of dysfunctions in the central nervous system. There is just no other location in the human body in which the deep seated, archaic regulatory units affected in ME/CFS are to be found. For us, ME/CFS 
is not a dysfunction that ALSO affects the CNS, it is a dysfunction that is DRIVEN by the CNS. This "central" explanation also covers the general mitochondrial dysfunction seen in ME/CFS, the muscular dysfunction and the "enigmatic cardiovascular situation in ME/CFS - "peripheral" as all these features may appear, they all can be explained as consequences of central regulatory dysfunctions. Bluntly, in an "Occam's razor" investigation, there is just no need for a "peripheral" explanation. Also, the "central" hypothesis is also the only explanation that fits with the specific "coupling" of symptoms in ME/CFS (see [2.5]). And it is also the only explanation for the multi-trigger, threshold driven, delayed and prolonged stress response after exercise (see [3.0]).

Here, we need to add a disclaimer: This is a functional description of how the clinical cascade of ME/CFS may be best explained. This does NOT imply that influences outside the CNS may not play a role in ME/CFS or may not be appropriate targets for therapeutic interventions. Similarly, the "central" argument does also not imply that there may not be more profound, general processes that inaugurate, prime, sustain or otherwise influence this central pathological matrix (to be revisited below).

\section{Conclusion 4}

The pathophysiology of ME/CFS affects completely disparate physiological processes. It is a "dauer state" and it is "sustained arousal" - at the same time. It is vagal dysfunction and sympathetic dysfunction - at the same time. It is adrenergic stimulation and adrenergic failure - at the same time. It is hyperarousal and under arousal - at the same time (for details on the "paradoxical" nature of ME/CFS, see [4.11]). ME/CFS just does not fit into any single regulatory unit. We therefore doubt that what is broken in ME/CFS can be found in a single brain nucleus (like a "fatigue nucleus") or a single transmitter system or a single receptor system. We rather suggest that the core pathology of ME/CFS is a diffuse one that affects a matrix that contributes to many regulatory units and spans many regulatory levels.

\section{Conclusion 5}

The pathogenetic processes of ME/CFS, fundamental and possibly pseudo-adaptive as they are, must involve the neuroglial matrix of the CNS. The astroglial compartment (which works in close cooperation with microglia and, evidently, the neurons) has to be considered the functional basis of the deep rooted, archaic regulatory circuits affected in ME/CFS. Indeed, the astroglial compartment is the only tissue which is simultaneously involved in the regulation of brain perfusion, of the bloodbrain-barrier, of neuroinflammation, of the innate immune system, of the basic circuits involved in autonomous functioning and the stress response. It is also the only tissue that at the same time influences mood, motor functions, sensory gating, memory and cognition. To me, pathology propagated along the astroglial tracts of the CNS is the best explanation for the concomitant mess-up of basic "metazoan", "limbic" and higher mammalian functions typical of ME/CFS.

These conclusions give rise to several questions: 
Question 1: If it is all in the CNS: may there be a part of the CNS that can be considered the tectonic hotbed of the ME/CFS pathology?

Question 2: With so many pathogenetic links clearly identified (including hypoperfusion, inflammation, abnormal adrenergic signaling etc.) - with which link could the pathogenetic chain in ME/CFS start?

Question 3: Which events may have triggered the whole process? What may be the initiating event of ME/CFS?

\section{Question 1: If it is all in the CNS: may there be a part of the CNS than can be considered the tectonic hotbed of the ME/CFS pathology?}

From the evidence base presened above there is some evidnce that the epicenter of the CNS dysfunctions leading to the clinical messup of ME/CFS is the brainstem. Again, this is not to say that the processes happening there may not be influenced or activated by processes happening elsewhere. And this is also not to say that the pathology of ME/CFS is restricted to the brainstem - it clearly is not, it is being propagated throughout many other parts of the brain (and finally, to the periphery, of course). Rather, we consider the brainstem the starting point of the pathophysiological cascade - this is where the first links of the pathogenetic chain take action (whatever they may be, see below).

From a basic physiological venue, the homeostatic and autonomous functions that are clearly disturbed in ME/CFS all rely on functional units located in the brainstem (for details, see [4.10]). Similarly, the brainstem is exactly the brain region which may influence and regulate the conspicuous "peripheral" features of ME/CFS, including misery perfusion, the enigmatic cardiovascular situation (for details see [4.2]), inflammatory processes, temperature and pain regulation as well as exercise tolerance (for details, see [2.5]). Plus, the brainstem is densely packed with mast cells, another possible pathogenetic link in ME/CFS (see [4.11]).

These theoretical considerations fit with the study record on ME/CFS patients. In all and any ME/CFS patient examined to this avail so far, blood flow in the brainstem or blood flow regulation (coordinated by brainstem nuclei) have been shown to be abnormal (for details, see [4.8]). Also, in the light of the clearly documented large numbers of clinical ME/CFS cases in patients with hypermobility syndromes and/or neuro-orthopedic conditions, it is plausible to speculate that indeed the lower brainstem may be the pathological hotbed of ME/CFS (for a thorough substantiation of this argument, see [5.0]). This may be even more credible as several research groups in their pathogenetic models of ME/CFS have implicated several of the regulatory centers in this region as possible starting points of more comprehensive upstream pathology (including the nucleus of the solitary tract, the dorsal motor nucleus of the nervus vagus and the reticular activating system including the locus coeruleus) ${ }^{5,190,193}$ (for details in this review, see [4.10] and [4.11]). Some of these regulatory centers have also been implied from other research teams who have identified abnormal baroreceptor and/or chemoreceptor functions as core feature in ME/CFS in their experimental work 
(for details, see [4.8]). Other researchers have delineated pathological processes in nuclei and brain circuits functionally closely related and influenced by the brainstem, including the basal ganglia, the suprachiasmatic nucleus, the paraventricular nucleus of the hypothalamus, ${ }^{7}$ or generally the limbic system. ${ }^{413}$ All these thoughtfully elaborated hypotheses find a common denominator if the profound regulatory input into these nuclei and circuits from the brainstem is being considered. Indeed, several teams have delineated the apparently inadequate communication in ME/CFS between the brainstem and upstream regions like the hypothalamus, hippocampus and prefrontal cortex - here again, the assumption of the brainstem as the tectonic epicenter may help explain the findings of widespread dysfunctions in other regulatory areas of the brain. ${ }^{5,110}$

\section{Question 2: With so many pathogenetic links clearly identified in ME/CFS research (including hypoperfusion, inflammation, abnormal adrenergic signaling etc.) - with which link could the pathogenetic chain in ME/CFS start?}

Identifying a dysfunctional brainstem as the topographical epicenter for all ME/CFS cases does not yet explain the pathophysiological chain of events at work (which may or may not be different in different subgroups of ME/CFS, a question which we need to revisit). This paper has listed many of these links, including:

- "mechanical" influences - which, as we have seen (see [5.0]) may not only be "mechanical" at all but may include effects on perfusion, inflammation, the innate immune system, lactate production, mast cell function, and possibly second messaging and CNS signaling in general.

- causes related to cerebral blood flow (i.e. hypoperfusion, hyperperfusion, dysregulated perfusion - if related to arterial or venous dysfunction, or related or unrelated to intracranial hypertension)

- causes related to inflammation or immune stimulation - if directly related to inflammatory mediators (including from activated mast cells) or related to autoimmunity (with or without effects on cellular or receptor/signaling functions)

- other causes, including abnormal signaling from vagal dysfunction or abnormal endo- or paracrine signaling (for example, dysfunctional signaling in the adrenergic, the CRF-ergic or the brain-RAS system may possibly cause fundamental dysregulation of brainstem centers or their connectivity)

From what we have seen, all these factors are plausible, but how and in which sequence may they be connected - and may they be so in all the subsets of ME/CFS?

\section{In search of a common denominator}

All ME/CFS cases meeting the acknowledged diagnostic criteria share the same clinical facets. But do they share the same pathophysiological pathways? Take the post-infectious form of ME/CFS and contrast it with the sizable number of ME/CFS cases related to neuro-orthopedic or connective tissue disorders (see [5.0]): What may be their common pathophysiological denominator if we consider the 
links presented above? May there be one sequence of pathological events that may plausibly explain ALL etiotypes?

Let us go along the list presented above:

- Mechanical strain alone has to be taken off the list as a potential common denominator as it clearly doesn't fit with the post-infectious cases. Intracranial hypertension also appears to be a secondary factor as it is not seen universally in ME/CFS and may be more prevalent in the "hypermobile" and the "neuro-orthopedic" cases of ME/CFS than in the post-infectious cases (I am judging this from the different prevalences found in different ME-CFS case-mixes). ${ }^{342,343}$

- Brain perfusion issues, however, seem to be a plausible candidate as a common denominator both from pathophysiological considerations and from the study record (which found evidence of cerebral perfusion defects in ALL the patients studied, for details, see [4.8]). This explanation also may fit well with the mechanical cases as well as with the post-infectious cases; after all, the brain dysfunctions seen in both cases may well be perfusion related (for details, see [5.0]).

- However, this is not to say that perfusion problems have to be considered the first link in the pathogenetic chain as clearly, they may themselves be caused by other pathomechanisms. There are indeed several plausible candidates for upstream causes: the perfusion problems may be a consequence of neuroinflammation (see [4.1]). Or they may be triggered by autoimmune processes which may for example affect targets in the chemo- or baroreceptor circuits or in other circuits linked to brain perfusion (e.g., adrenergic receptors, b-RASreceptors or other receptors related to the epinephrine release or function).

- The same central, yet ambiguous role can be described for the inflammatory link on the list. Neuroinflammation, for instance, clearly may be another possible common denominator for all ME/CFS cases. After all, the pathogenetic role for neuroinflammation in ME/CFS is plausible and at least in part borne out by evidence (see [4.1]), and this holds true for both the post-infectious cases and the "mechanical" cases where neuroinflammatory processes may also play a role (see [5.0]). But here again, nothing is said about the position of this link in the chain of events: does neuroinflammation cause perfusion problems or vice versa?

- For us, this may be a futile question because there is good evidence that perfusion and inflammatory processes act in union and may rightfully be considered a package. For one, deranged blood flow and ischemia give rise to neuroinflammation; similarly, neuroinflammation and the associated edema and mitochondrial and cellular dysfunction give rise to endothelial dysfunction and deranged perfusion. Clearly, from a pathophysiological standpoint, perfusional and inflammatory changes may be two sides of the same coin.

As a plausible summary I would therefore suggest that a - to be further defined - "inflammatoryperfusional package" may be dysregulated in all cases of ME/CFS and that this may indeed be the common denominator in all cases of ME/CFS. Clearly this "package" seems to be triggered by different initiating influences in the different subsets, yet, for me, they feed into the same central pathological hub in ME/CFS.

Revisiting the case for a central role of neuroglia 
It is highly plausible that this pathogenetic "package" corresponds to a "functional package" in human biology - the astroglial matrix of the CNS. ${ }^{100}$

Indeed, together with its partner tissue, the microglia, this is the only tissue in the human body which is physiologically both part of the innate immune system AND part of the vascular-endothelial unit (which also constitutes the blood-brain barrier) AND part of the functional neuronal matrix (for details, see [1.0]). This is a truly "neuro-immune" tissue. I am convinced that we have to take this multi-functional matrix seriously, and therefore, at the risk of repeating myself, I want to summarize some of the physiological functions covered by the astroglial compartment:

- This tissue, in cooperation with microglia, is heavily involved in both acute and chronic inflammatory responses and at the same time has been shown to be tonically activated in response to all kinds of stress signals. Indeed, ALL the pathological core features of ME/CFS are consistent with changes in glial activity, including cerebral hypoperfusion, neuroinflammation and other inflammatory changes, exaggerated stress response, a leaky blood-brain-barrier, sympathetic activation and autonomous dysfunction.

- This tissue with its unique anchorage in both the vascular system, the innate immune system and its direct cooperation with neurons may also explain why perfusion defects, inflammatory responses and neuronal dysfunction so often appear virtually inseparable in ME/CFS. Indeed, if one summarizes the current research evidence the big themes ALWAYS seem to be blood flow issues, inflammation and an abnormal immune response - and they ALWAYS seem to be related.

- What is more, there is no other functional compartment which may be able to explain the enigmatic, peculiar form of autonomic dysfunction that has been found in all and any ME/CFS patient accordingly studied so far: positional hypoperfusion with a credible involvement of dysfunctional baroreception - it is again the astrocyte compartment that regulates the latter circuit (for details, see [4.8]).

- The same holds true for the sensory hyperexcitatory aspects of the disease (or, more generally, the picture of excitotoxicity so typical of ME/CFS): here, astrocytes, with their unique role in the regulation of the synaptic availability of glutamate should be considered central players too.

- The astroglial compartment also may explain why such disparate etiotypes as the "mechanical" and the "post-infectious" cases may end up in the same clinical phenotypes: after all, glia is a compartment that has clearly been shown to be activated by both repetitive mechanical strain and by immune processes (for details, see [5.0]).

- Moreover, with reference to the possibly very central role that a dysfunctional vagal nerve may play in ME/CFS (a theory that I strongly believe in and in which I also place many hopes in regards to therapy) - any dysfunctions of the vagal nerve can only be understood in the light of the central role of astrocytes (for details, see [4.10]).

- The same holds true for the emerging role of mast cells in ME/CFS pathology (see [4.11]) they have been identified as possible "partners in crime" of astroglia.

- Also, the astroglial tissue may explain the conspicious implication in the pathogenetic process of ME/CFS of a wide range of topographical and functional CNS units, spanning all known transmitter and receptor systems: upon activation, glial cells produce their own inflammatory mediators through which the inflammatory response is transduced to nearby glial tissue but also to distant brain locations through the whole neural connectome of the 
brain - which, as it happens, connects brain areas that all have been implicated by different research groups in the pathology of ME/CFS

- And finally, and possibly most importantly, it needs to be remembered that astrocytes and microglial cells are unique among all other cellular compartments in their flexible response to stress - they are able to change their functional state profoundly in response to homeostatic or allostatic influences. And they are able of forming "stress memories" of sorts: following pro-inflammatory stimulation these cells remain in an activated state and thereby become hyperresponsive to any form of subsequent stimulation: primed astroglia reacts on a different threshold level than quiescent astroglia. Also, once repetitively activated, the activation threshhold may decrease and with it the ability of the astrocyte to revert to normal function. This indeed may be the only tissue that may explain the waxing and waning picture of ME/CFS and its unique clinical feature of post-exertional malaise.

For me, the evidence for a central role of the glial compartment is overwhelming. This does NOT imply that we should not also focus on abnormal signaling, receptor dysfunction, autoimmunity or other pathophysiological influences - we should. Here, fibromyalgia, the "sister disease" of ME/CFS may be a case in point. In fibromyalgia, the glial compartment in the dorsal root ganglia has now to be considered the prime candidate as the central pathological hub, and there is overwhelming evidence that it may be stimulated by an autoantibody. ${ }^{262}$ May a similar process be at work in ME/CFS? We don't know, but this is possible (even if the process in ME/CFS more plausibly may be directed against glial targets in the CNS). What we do know about glial activation is that this process easily extends to other areas of the glial compartment, no matter how it is being triggered. ${ }^{414}$ And we know another thing: glia activation tends to end up in a self perpetuating process (see [1.0]).

\section{Does ME/CFS represent connectome failure?}

This may be even more plausible in the light of our emerging understanding of the functional matrix of the brain (for a review, see ${ }^{415}$ as well $\mathrm{as}^{416}$ ). Neuroscientists used to try to understand brain function and dysfunction starting from topographical units (brain areas or brain nuclei) to which they assigned specific functions. The hope was that by tracing back pathology to these specific areas one may find "the broken piece" and fix it. A similar approach was later pursued with neurotransmitters and their receptors - a certain disease, like depression, was then coined as a disease in which a certain transmitter (or receptor) system was dysfunctional.

Everyone who has followed ME/CFS research for a while is familiar with a similar narrative. There have been ever new hypotheses as to which topographical unit may explain ME/CFS, an approach in which variably the locus coeruleus, the amygdala, the paraventricular nucleus, the suprachiasmatic nucleus or the vagal centers of the brainstem have been implied as "the broken pieces" of ME/CFS. Even a "fatigue nucleus" has been suggested as "the" area of concern in ME/CFS. ${ }^{417}$ Likewise, transmitter systems have been identified as dysfunctional, and variably dysfunctions in the dopaminergic transmission, the serotoninergic transmission, the glutaminergic transmission, or the adrenergic transmission have been suggested as the biochemical basis of ME/CFS. Similarly, specific receptor dysfunctions have been implied as the pathological basis of ME/CFS.

And indeed, ALL these topographical or biochemical explanations make physiological sense and none of them can be refuted. As a matter of fact, studying any of these explanations leaves the reader 
with a deep sense of scientific satisfaction. Yet, the obvious disparity and contrariness of these explanations clearly demonstrates that these may only be pieces of a wider, and possibly very complex picture.

Neuroscientists are now working on a brain model that tries to address this complexity. They now understand the brain as a functional matrix of interconnected pathways forming multiple large-scale functional networks (called resting-state networks, like for instance the default mode network). The analysis of the functional connectivity of these networks has identified sets of regions that can be seen as essential "hubs" for efficient neuronal signaling and communication. While these hubs are embedded in specific anatomical locations, they have functional roles across a wide range of cognitive and affective tasks and are part of a multitude of transmitter systems. Pathology may arise from dysfunction or disconnection within these brain hubs through which they lose their local connectedness and short path length (indicative of global integration). It has been suggested that such "connectivity hub overload" or "hub failure" may be a potential final common pathway of several neurological diseases. Indeed, such altered functional connectivity has now been identified in a wide variety of CNS diseases, including anxiety, posttraumatic stress disorder, anorexia nervosa or depression. It is plausible that similar changes may possibly also apply to ME/CFS, where functional connectivity issues have also been identified in resting state and task functional MRI data. ${ }^{5}$

Interestingly, and in support of the general hypothesis laid out in this working paper, there is evidence that astrocytes may be the decisive players in the energy hungry neuronal connectome of the brain; indeed, their dysfunction may be at the beginning of connectivity hub failure. ${ }^{67,418}$ So here again, we may be back to a tissue that reaches across and beyond specific brain nuclei, transmitter and receptor systems: the astroglial compartment.

\subsection{The big question: Which event(s) may trigger the whole process? What may be the initiating event of ME/CFS?}

The evidence that I have revisited suggests that ME/CFS may be a disorder in which glial activation possibly triggered by a multitude of noxious events, including but not limited to infections - DOES NOT STOP FOR SOME REASON, leaving the glial compartment primed for further insults and hyperreactive to stress. The latter may explain the lingering character of the disease as well as its exacerbations by stressful events.

What may be the reason(s) for this "does not stop for some reason"? Many hypotheses have been forwarded, including persistent infection of the brain or of the vagus nerve (e.g., from herpes viruses), a persistent immune reaction against viral or microbial protein remnants, an autoimmune reaction against functional units of the body (e.g., GPC receptors), reactivation of neurotropic pathogens such as EBV and other herpesviruses, or neuroinflammation sustained through repetitive mechanical strain or repetitive immunological insults. Others have suggested epigenetic influences through which some gene transcription (e.g., for genes involved in the stress response) has become dysregulated and "does not stop for some reason". Again, others have suggested a "metabolic trap" that has snapped shut for some reason. Or suspect influences that during a normal, physiological 
"sickness" response may have resulted in "connectivity hub overload" in the CNS of some susceptible individuals, resulting in a rewiring of the brain connectome (for details, see below).

All these influences have in common that they may not be mutually exclusive - and that they may all end up in a pathologically activated neuroglia.

There are good reasons to argue that the post-infectious cases of ME/CFS may be best understood as a maladaptive sickness response (no matter if it is triggered by microbes directly or by microbial remnants or by reactivated endogenous microbes or by parainfectious autoimmune processes). To understand how the sickness response may turn maladaptive a lot may to be learned from the depression model discussed by David Poon et al. ${ }^{50}$ and recently, with regards to Long Covid, also by Mondelli and Pariante. ${ }^{419}$ Poon et al. discuss the observation that during Interferon-alpha therapy for malignant melanoma all patients develop standard sickness behavior via the classical "infectious disease" inflammatory pathways: they withdraw socially, they become fatigued, anhedonic and anorexic, they sleep more etc. But a subset of the patients treated with TNF-alpha goes on to develop overt clinical depression. Interestingly, this development may play on a different inflammatory pathway - the IDO pathway (for details, see [4.6]). So there may be some susceptibility influence that in some individuals may lead to a specific dysfunction or runaway process in the adaptive sickness response. Here, immune events like reactivation of endogenous microbes or a genetic predisposition may come to mind. Indeed, there are some suggestions from patients with infectious hepatitis treated with IFN-alpha that one of these factors may be a genetic one as patients with genetic variants (here, for IL-6, interferon gamma but also for the IDO promoter gene) have been shown to be at greater risk of developing clinical depression during IFN-alpha treatment. ${ }^{420}$ In this context, the hereditary component of ME/CFS may be worthwhile remembering. ${ }^{421}$

A similar point is made by Mondelli and Pariante who point to the fact that peripheral inflammatory stimulation clearly is able to induce central neuroinflammation, yet with a twist. For one, this seems to be an exceptional response as indeed, most peripheral infections remain localized. Second, in experiments using peripheral immune challenges like IFN-alpha there is often no dose-response dynamic to be observed between the severity of the inflammation and intensity of CNS symptoms like fatigue - especially if the symptoms start only weeks after the initial immune challenge (a similar delay in symptom onset is reported by many ME/CFS patients, and now also in PASC; ;22 also, in $\mathrm{ME} / \mathrm{CFS}$ in general there seems to be little correlation between severity of the triggering infection and the severity of ME/CFS that follows). These features may again point to an abnormal sickness response as core element in the inception of post-infectious ME/CFS, where, in the words of Mondelli and Pariante, "the individual sensitivity to immune activation might be more important than the absolute levels of immune activation per se. ${ }^{419} \mathrm{I}$.e, the discriminatory feature between those who recover after a infection induced sickness response and those who do not may to be found in the fact that, in the latter, the peripheral inflammatory stimulation gets a) transduced into the CNS and $b$ ) there becomes sustained by additional immunological mechanisms "even when the initial trigger from peripheral immune activation has resolved." Here, Mondelli and Pariante suggest "central processes involving brain/microglia response". Some of these additional mechanisms that may confer susceptibility to some people may include a higher likelihood of glial activation, a higher likelihood of BBB disruption allowing crosstalk between peripheral immune processes and those in the CNS as well as the activation of the kynurenine pathway. ${ }^{419}$ 
This is not to say that these are the only mechanism that may throw off the adaptive sickness response in some individuals, yet, the concept of vulnerability seems to be an important one. Also, the IDO pathway referred to in both of the hypotheses presented, has been implicated as a pathogenetic possibility in ME/CFS, too (see [4.6]). Clearly, it may be a valuable addition to the research agenda to identify factors which may derail the adaptive systemic inflammation-induced sickness response in some individuals.

\subsection{COVID-19 to the rescue}

The current research on "Long Covid" (Post-Acute Sequelae of Covid, PASC) may clarify some of these hypotheses - at least with respect to the post viral cases of ME/CFS. At least, this is the hope of many ME/CFS patients, myself included. As of now, I see several noteworthy developments and pieces of evidence:

- COVID-19 seems to be among the viruses with a high likelihood of leaving chronic debilitation in its wake. A subset of PASC patients clearly falls into the ME/CFS category because of the typical persistence of symptoms ${ }^{423}$ and the typical syndromatic picture of psychomotor slowing, PEM, hypersensitivity to light and sounds and severe fatigue coupled with mood dysfunctions like anxiety. ${ }^{424}$ This group may also share the typical gender and age predilection seen in ME/CFS. ${ }^{425}$ Clearly, this PASC group (now sometimes calles "neuroPASC") should be studied separately.

- There are quite a few reports of a delayed onset of PASC after the initial SARS-CoV-2 infection: "I felt better - and then after a few weeks the long haul began." A recent analysis of PASC courses confirms that PASC symtoms seem to develop within 6 weeks after the acute infection, ${ }^{422}$ which may fit well with the time frame needed for the inception of autoimmune processes and/or reactivation of endogenous microbial reservoirs.

- The development of PASC seems be independent of initial symptoms, severity of disease ${ }^{426}$ and even the level of peripheral inflammatory markers ${ }^{424}$ - again, an interesting finding.

- It has been shown that viral persistence (or the persistence of viral material) in the periphery is possible after SARS-CoV-2 infection, especially in the GI tract, ${ }^{427}$ but also possibly in monocytes and Kupffer cells of the liver ${ }^{428}$ and elsewhere. ${ }^{429}$ The same has been suggested for the CNS where direct invasion of viruses into the astroglial compartment has been shown. ${ }^{430,431}$ It has also been shown that entry into the nervous system space may happen through capillary pericyte infection by SARS-CoV-2. ${ }^{432}$ The fact however that SARS-CoV-2 infection is not consistently found in COVID-19 patients with neurological symptoms may suggest a transient nature of CNS infection. ${ }^{433}$ Also, the degree of neuroinvasion by SARSCoV-2 does not seem to correlate with the severity of neuropathological changes. ${ }^{419} \quad A$ study of vaccine responses in PASC patients has been interpreted as support for viral persistence in the periphery. ${ }^{434}$ So at this point viral persistence in either the periphery or the CNS are plausible contributors to PASC inception, at the same time there is no evidence that viral persistence may be a prerequisite for the development of the ME/CFS-like cases of PASC (given the fact that SARS-CoV-2 has been shown to use the olfactory nerve to enter the CNS it would be interesting to find out if loss of smell is associated with the development of Long Covid).

- It has been suggested that EBV reactivation (based on positive titers for EBV early antigendiffuse IgG or EBV viral capsid antigen IgM) may be a common occurence in patients with 
PASC; ${ }^{435}$ however, it is not known if this is a cause of PASC or may reflect immune dysregulation going along with PASC. Indeed, it has been shown that any neuroinflammatory illness may cause EBV reactivation, a conundrum which also applies to ME/CFS research (in $\mathrm{ME} / \mathrm{CFS}, \mathrm{EBV}$ reactivation has also been suggested to play a pathogenetic role, ${ }^{242}$ and upregulation of EBV virus induced gene 2 (EBI2) has been described, which codes for a GPC receptor that may play a role in neuroinflammation and other CNS functions)..$^{1192}$

- Long Covid clearly seems to be marked by inflammatory changes, as evidenced by elevated serum inflammatory cytokine profile in symptomatic Long Covid patients that was maintained at 8 months post-infection and was not observed in asymptomatic COVID-19 survivors. ${ }^{436}$ Recently it has been shown that development of PASC was associated with immune activation as evidenced by the persistence of increased levels of TNF-alpha and IL$6^{434,437}$ (in the latter study an enhanced IL- 6 production by CD8+T cells is suggested as a pathogenetic link in PASC development).

- Very much like ME/CFS patients, post-COVID-19 patients without cardiopulmonary disease have been shown to have a clearly abnormal exercise capacity with a marked reduction in peak VO2, impaired systemic oxygen extraction and an exaggerated hyper-ventilatory response during exercise - all findings cannot be explained by cardiac limitations. ${ }^{438}$

- There may be microbiome changes in some PASC cases as supported by the observation of increased levels of 3-indole acetic acid which implies a microbiome functionality shift. ${ }^{313}$

- It seems that the activation of the innate as well as the adaptive immune system may play a central role in PASC, and maintenance of long-lasting inflammation and immune activation after COVID-19 infection has been well documented. ${ }^{436}$ Elevated IL-1beta (as a possible marker for innate immune system stimulation) seems to be a consistent signal in studies of PASC. ${ }^{439,440}$ Also SARS-CoV-2 vaccination seems to have given (if only temporarily) respite to some of Long Covid sufferers ${ }^{441,442}$ _ however, this is controversial as one analysis do not support this. ${ }^{433}$ Improvements after vaccination may somewhat reminiscent of the very marked success of the staphylococcal vaccine Staphypan in ME/CFS (unfortunately withdrawn from the market by the company many years ago). ${ }^{246,247}$ PASC patients also exhibit broad dysfunction in T cell memory generation and aberrantly elevated T cell responses to SARS-CoV-2 mRNA vaccines. ${ }^{434}$

- Autoimmunity seems to be a big theme in Covid, with more than 100 autoantibodies detected in Covid patients, ${ }^{260}$ some of which are to be found in the cerebrospinal fluid and pertain to neuronal targets. ${ }^{444}$ People with autoimmune diseases appear twice as likely to develop long COVID, ${ }^{445}$ and possibly may also have more severe long-COVID symptoms. Also, ANA titres have been shown to be elevated in the subset of PASC patients with neurocognitive symptoms. ${ }^{423}$ While the level of some of these autoantibodies subside over time, others persist. So there is a potential that in the aftermath of the infection autoimmune targets are being primed, which indeed seems to happen in PASC. ${ }^{236,446}$ The autoimmunity explanation would also fit quite well with the time lag typically reported between the acute infection and the beginning of PASC symptoms which show a very conspicious rise in prevalence within the first 6 weeks after the acute infection ${ }^{422}$ - similar to the time course reported for the development of autoimmune manifestations like GuillainBarre-Syndrome after SARS-CoV-2 vaccination. ${ }^{447}$ As of now, however, the role of the autoantibodies in the disease process of PASC remains ill defined, ${ }^{236}$ even if a first single-case experimental therapy with the auto-antibody scavenger BC 007 has apparently shown promising results in a PASC patient ${ }^{266}$ (now confirmed in two other PASC patients). ${ }^{448}$ 
- Microvascular injuries seem to play a role in Covid ${ }^{449}$ and there is some evidence of damage to peri-vascular cells ${ }^{432}$ as well as capillary structural changes. ${ }^{450}$ Also, there is evidence for BBB disruption in acute Covid with evidence of extravasation of serum through leaky small vessels in the brain. ${ }^{451}$ There is no evidence of similar processes in PASC as of yet (but some earlier evidence of punctate hyperintensities in the brain of ME/CFS patients; ${ }^{349}$ also, there are theories that extravasation of fibrinogen may stimulate the innate immune system and initiate neuroinflammation or autoreactivity as postulated in a hypothesis of Complex Regional Pain Syndrome). 452,453

- Endothelial dysfunction with an activated clotting cascade has been found in Long Covid patients, and these findings seem to correlate with severity of fatigue and reduced physical fitness. ${ }^{454,455}$ Another study has found evidence for the presence of persistent anomalous (amyloid) microclots and a pathological fibrinolytic system in Long Covid patients. In these microclots, high levels of various inflammatory molecules including fibrinogen and alpha(2)antiplasmin have been found and there are trial that indicate that apharesis of these microclots could improve symptomatology. ${ }^{455}$

- Another "vascular" hypothesis has focused on the possible role of non-classical monocytes rendered dysfunctional and long lived from persistent intracellular SARS CoV-2 S1 protein. ${ }^{456}$ The high CCL5/RANTES levels secreted by these monocytes may foster endothelial dysfunction and inappropriate vasodilation. The proponents of this hypothesis claim to have successfully treated "thousands" of Long Covid patients with a therapy including an CCL5 antagonist. ${ }^{457}$ (However, it needs to be remembered that CCL5 plays a complex and diverse biological role that includes astroglial reactivity, ${ }^{458,459}$ regulation of T-cell traffic into the Central Nervous System, ${ }^{460}$ and may also contribute to reactivation of endogenous infections in the CNS and other immune procecesses relating to neuroinflammation). ${ }^{458(p), 459,461}$

- It seems that autonomic dysfunction may explain some of the PASC symptoms. ${ }^{462}$ Also, impaired parasympathetic modulation of HRV has been observed after COVID-19 infection through 6 months post-discharge. ${ }^{463}$

- The activation of stress response programs in PASC patients has been suggested in a study of immune parameters based on the expression of AP- 1 and DDIT4 genes ${ }^{439}$

- Abnormal brain perfusion and/or hypometabolisms have been demonstrated both in adult and in pediatric PASC patients. ${ }^{464,465}$

- It seems that neuroinflammation with activation of innate and adaptive immune cells plays a role in acute Covid $19^{466,467}$ but also in PASC, as evidenced by gene ontology network analyses which highlights neuroinflammatory responses. ${ }^{468}$ It has been suggested that neuroinflammation may influence neurological sequelae in PASC by altering $\mathrm{nEV}$ proteins (neuronal-enriched extracellular vesicle proteins). ${ }^{468} \mathrm{~A}$ biobank study in the UK comparing brain scans before and after SARS-CoV-2 infection gives evidence of significant longitudinal differences in a network of regulatory brain regions involving the anterior cingulate cortex and orbitofrontal cortex, as well as the entral striatum, amygdala, hippocampus and parahippocampal gyrus - all areas that have also been implicated in ME/CFS neuroimaging pathology and may possibly be a reflection of neuroinflammation. ${ }^{469}$

- Mast cell activity has been frequently implied in acute Covid and lately it has been shown that symptoms of mast cell activation may also be present in Long Covid. ${ }^{470}$ Also, there are reports of antihistamine therapy being effective in $\mathrm{PASC}^{295}$ - some potential modes of action are emerging. ${ }^{471}$ 
- Finally, there is now abundant evidence that the neuroglial compartment is diseased or activated in COVID-19, a pathological feature that has been demonstrated in animal models of coronavirus infections, where the neurovirulence of the virus correlates with its ability to induce proinflammatory cytokines in astrocytes and microglia. ${ }^{472}$ SARS-CoV-2 has been shown to display a strong tropism for developing human cortical astrocytes ${ }^{473}$ (for a very lucid summary on the potential role of astroglia in Covid 19, see ${ }^{474}$ ), and indeed, astroglioses has been shown to be present in the vast majority of brain specimens of COVID-19 victims, with microglia reactivity and infiltration of neurotoxic T-cells mainly seen in the brainstem and cerebellum. ${ }^{475}$ Also, glial activation seems to happen during acute COVID $19^{467}$ as also evidenced by the fact that plasma concentration of the astrogliosis marker GFAP are significantly increased in patients with moderate to severe Covid. ${ }^{476} \mathrm{Glial}$ activation during acute COVID-19 may be especially prominent in the brainstem. ${ }^{477}$ However, in a longitudinal analysis of patients developing PASC symptoms after acute COVID-19 the originally elevated GFAP levels normalized and were not indicative of a Long Covid course. ${ }^{478}$ It has been suggested that during SARS-CoV-2 infection the performance of astrocytes and microglia may be especially critical for the protection of brain areas devoid of a blood-brain barrier such as the circumventricular organs, where circulating inflammatory mediators may more easily initiate inflammatory processes. Other researchers have pointed out that downregulation in ACE2 level induced by SARS-CoV-2 could have detrimental effects through dysregulation of the astrocytes in the brain RAS system, resulting in reduced brain blood flow and poor cardiovascular control. ${ }^{149}$

\section{What is the evidence for neuroglial activation in PASC?}

A contributing role of a possibly activated astroglial compartment may appear plausible from the fact that increased IL-1beta levels have been observed in PASC, see above (IL-1beta has been shown to be able to activate astrocytes). It has now been shown in gene ontology network analyses that indeed the astroglial compartment may be activated in PASC as evidenced by upregulated genes for glial activation, astrocyte development, astrocyte activation and differentiation as well as microglial activation. ${ }^{468}$ In a study delineating the persistent immune activation after acute SARS-CoV-2 infection MCP-1 (CCL2) has been identified as one of the most associative biomarkers associated with Long Covid ${ }^{436}$ (CCL2 has been shown to be involved in the activation and recruitment of microglial cells as well as in leucocyte trafficing across the BBB). ${ }^{479,480(\mathrm{p} 2)}$ Also, an analysis of the neuronalenriched extracellular vesicle protein cargo in patients with Long Covid has sketched a possible pathway to neuroinflammation in PASC by showing interrelated protein networks including HMGB1, "a ubiquitous nuclear protein that, when released extracellularly, promotes inflammation and cytokine release". Apparently, this nuclear protein can activate microglia, and, "when released via EVs into the periphery, can activate monocytes to promote further inflammation." In their gene ontology network analysis of 10 proteins "many functions around astrocyte and glial dysfunction and also glutamate receptor signaling stood out." ${ }^{468}$ On the other hand, GFAP levels do not seem to track with the development of Long Covid symptoms, which may argue against significant neuroglial activation. $^{478}$

As a possible route for the initiation of neuroinflammation it needs to be remembered that astrocyte/glial activation can also be a "mirror response" to cytokine signaling from systemic 
infections, transmitted, for instance, through the vagal nerve, for details, see [4.4]). Here, interestingly, it has been shown in a human organ model that direct SARS-CoV-2 exposure had no effects on BBB function, whereas the infusion of endothelial medium from infected alveoli was abke to cause BBB dysfunction and neuroinflammation, including brain endothelium disruption, glial cell activation and inflammatory cytokines release. ${ }^{481} \mathrm{Also}$, it is plausible that the widespread reactivation of latent EBV observed in PASC may play a role in the initiation of neuroinflammation, as it may go along with upregulation of genes central to the regulation of the innate immune system and CNS functions, ${ }^{191,192}$ (so far there is no corroborating evidence for this hypothesis, however).

There is now some evidence from the analysis of the transcriptome in COVID-19 patients that point to a role of peripheral immune processes playing a role in establishing neuroinflammation. ${ }^{482}$ In this analysis, the authors found no molecular traces of SARS-CoV-2 in the brain, but indications "that barrier cells of the choroid plexus sense and relay peripheral inflammation into the brain". In this process, the brain parenchyma apparently may become infiltrated with T cells - one of the pathomechanisms that promote neuroinflammation. Concomitantly, the neuroglia seems to be functionally transformed into an activated, pathological state: "We discover microglia and astrocyte subpopulations associated with COVID-19 that share features with pathological cell states that have previously been reported in human neurodegenerative disease" (and, as a possibly important addition from very recent research, in fibromyalgia - which seems to be another member of the "fatigue family" in which activated neuroglia seems to be of central importance). ${ }^{262}$

This explanation may fit well with imaging studies of PASC patients that have shown severely disrupted brain hyperconnectivity ("increased functional connectivity among regions of several resting-state networks" ${ }^{1387}$ ) - somewhat reminiscent of the findings of Barnden et al. in ME/CFS ${ }^{5}$ as well as hypometabolism in certain regulatory areas including the parahippocampal gyrus, thalamus, amygdala and, notably, brainstem and pons. ${ }^{391,465,483}$ Basically, here the same regions of interest emerge as in ME/CFS research. A focus on persistent brainstem dysfunction, partially also based on the hypothesis of lingering inflammation/astroglial activation has recently been proposed. ${ }^{484}$ In another hypothesis paper of how PASC may develop, ${ }^{170}$ it has been pointed out that in patients recovered from SARS-CoV-2 infection, microglia could remain primed via an innate immune memory transformation program mediated by epigenetic changes. ${ }^{485}$

So here again we may have every reason to focus our studies on the role of neuroinflammation and the neuroglial compartment. 


\section{References}

1. Komaroff AL. Inflammation correlates with symptoms in chronic fatigue syndrome. Proc Natl Acad Sci. 2017;114(34):8914-8916. doi:10.1073/pnas.1712475114

2. Wirth K, Scheibenbogen C. A Unifying Hypothesis of the Pathophysiology of Myalgic Encephalomyelitis/Chronic Fatigue Syndrome (ME/CFS): Recognitions from the finding of autoantibodies against ß2-adrenergic receptors. Autoimmun Rev. 2020;19(6):102527. doi:10.1016/j.autrev.2020.102527

3. VanElzakker MB. Chronic fatigue syndrome from vagus nerve infection: a psychoneuroimmunological hypothesis. Med Hypotheses. 2013;81(3):414-423. doi:10.1016/j.mehy.2013.05.034

4. Mueller C, Lin JC, Sheriff S, Maudsley AA, Younger JW. Evidence of widespread metabolite abnormalities in Myalgic encephalomyelitis/chronic fatigue syndrome: assessment with whole-brain magnetic resonance spectroscopy. Brain Imaging Behav. 2020;14(2):562-572. doi:10.1007/s11682-018-0029-4

5. Barnden $L R$, Shan $Z Y$, Staines DR, et al. Intra brainstem connectivity is impaired in chronic fatigue syndrome. Neurolmage Clin. 2019;24:102045. doi:10.1016/j.nicl.2019.102045

6. van Campen C (Linda) MC, Rowe PC, Visser FC. Two-Day Cardiopulmonary Exercise Testing in Females with a Severe Grade of Myalgic Encephalomyelitis/Chronic Fatigue Syndrome: Comparison with Patients with Mild and Moderate Disease. Healthcare. 2020;8(3):192. doi:10.3390/healthcare8030192

7. Mackay A, Tate WP. A compromised paraventricular nucleus within a dysfunctional hypothalamus: A novel neuroinflammatory paradigm for ME/CFS. Int J Immunopathol Pharmacol. 2018;32:2058738418812342. doi:10.1177/2058738418812342

8. Wyller VB, Eriksen HR, Malterud K. Can sustained arousal explain the Chronic Fatigue Syndrome? Behav Brain Funct. 2009;5(1):10. doi:10.1186/1744-9081-5-10

9. Saha AK, Schmidt BR, Wilhelmy J, et al. Red blood cell deformability is diminished in patients with Chronic Fatigue Syndrome. Clin Hemorheol Microcirc. 2019;71(1):113-116.

doi:10.3233/CH-180469

10. Schreiner $P$, Harrer $T$, Scheibenbogen $C$, et al. Human Herpesvirus-6 Reactivation, Mitochondrial Fragmentation, and the Coordination of Antiviral and Metabolic Phenotypes in Myalgic Encephalomyelitis/Chronic Fatigue Syndrome. ImmunoHorizons. 2020;4(4):201-215. doi:10.4049/immunohorizons.2000006

11. Naviaux RK. Metabolic features of the cell danger response. Mitochondrion. 2014;16:7-17. doi:10.1016/j.mito.2013.08.006

12. Wyller VB, Helland IB. Relationship between autonomic cardiovascular control, case definition, clinical symptoms, and functional disability in adolescent chronic fatigue syndrome: an exploratory study. Biopsychosoc Med. 2013;7(1):5. doi:10.1186/1751-0759-7-5

13. Harrington ME. Neurobiological studies of fatigue. Prog Neurobiol. 2012;99(2):93-105. doi:10.1016/j.pneurobio.2012.07.004 
14. Miller AH, Jones JF, Drake DF, Tian H, Unger ER, Pagnoni G. Decreased Basal Ganglia Activation in Subjects with Chronic Fatigue Syndrome: Association with Symptoms of Fatigue. PLOS ONE. 2014;9(5). doi:10.1371/journal.pone.0098156

15. Dobryakova E, Genova HM, DeLuca J, Wylie GR. The Dopamine Imbalance Hypothesis of Fatigue in Multiple Sclerosis and Other Neurological Disorders. Front Neurol. 2015;6:52. doi:10.3389/fneur.2015.00052

16. Yamamoto $\mathrm{S}$, Ouchi $\mathrm{Y}$, Onoe $\mathrm{H}$, et al. Reduction of serotonin transporters of patients with chronic fatigue syndrome. NeuroReport. 2004;15(17):2571-2574.

17. Hannestad $U$, Theodorsson E, Evengård B. $\beta$-Alanine and $\gamma$-aminobutyric acid in chronic fatigue syndrome. Clin Chim Acta. 2007;376(1):23-29. doi:10.1016/j.cca.2006.07.004

18. Marco. Glutamate - One More Piece in the Chronic Fatigue Syndrome (ME/CFS) Puzzle? The Neuroinflammatory Series Pt. II. Health Rising. Published February 15, 2013. Accessed June 21, 2021. https://www.healthrising.org/blog/2013/02/15/glutamate-one-more-piece-in-thechronic-fatigue-syndrome-mecfs-puzzle-the-neuroinflammatory-series-pt-ii/

19. Jennifer Brea. Jennifer Brea. Accessed June 13, 2021. http://www.jenniferbrea.com

20. Roerink ME, Lenders JWM, Schmits IC, et al. Postural orthostatic tachycardia is not a useful diagnostic marker for chronic fatigue syndrome. J Intern Med. 2017;281(2):179-188. doi:10.1111/joim.12564

21. Hvidberg MF, Brinth LS, Olesen AV, Petersen KD, Ehlers L. The Health-Related Quality of Life for Patients with Myalgic Encephalomyelitis / Chronic Fatigue Syndrome (ME/CFS). PLOS ONE. 2015;10(7):e0132421. doi:10.1371/journal.pone.0132421

22. Saura J. Microglial cells in astroglial cultures: a cautionary note. J Neuroinflammation. 2007;4(1):26. doi:10.1186/1742-2094-4-26

23. Thiele DL, Kurosaka M, Lipsky PE. Phenotype of the accessory cell necessary for mitogenstimulated $T$ and $B$ cell responses in human peripheral blood: delineation by its sensitivity to the lysosomotropic agent, L-leucine methyl ester. J Immunol Baltim Md 1950. 1983;131(5):2282-2290.

24. Massimo B, Laura F, Carla A, Carla M, Stephen DS, Pietro G. Astrocyte-Microglia Cooperation in the Expression of a Pro-Inflammatory Phenotype. CNS Neurol Disord - Drug Targets. 2013;12(5):608-618.

25. Marina N, Christie IN, Korsak A, et al. Astrocytes monitor cerebral perfusion and control systemic circulation to maintain brain blood flow. Nat Commun. 2020;11(1):131. doi:10.1038/s41467-019-13956-y

26. Mastitskaya S, Turovsky E, Marina N, et al. Astrocytes Modulate Baroreflex Sensitivity at the Level of the Nucleus of the Solitary Tract. J Neurosci. 2020;40(15):3052-3062. doi:10.1523/JNEUROSCI.1438-19.2020

27. Bisht K, Okojie KA, Sharma K, et al. Capillary-associated microglia regulate vascular structure and function through PANX1-P2RY12 coupling. bioRxiv. Published online August 4, 2021:2021.08.02.454769. doi:10.1101/2021.08.02.454769 
28. Hösli L, Hösli E. Receptors for dopamine and serotonin on astrocytes of cultured rat central nervous system. J Physiol (Paris). 1987;82(4):191-195.

29. Kruijver FPM, Balesar R, Espila AM, Unmehopa UA, Swaab DF. Estrogen-receptor-beta distribution in the human hypothalamus: similarities and differences with ER alpha distribution. J Comp Neurol. 2003;466(2):251-277. doi:10.1002/cne.10899

30. Langub MC, Watson RE. Estrogen receptor-immunoreactive glia, endothelia, and ependyma in guinea pig preoptic area and median eminence: electron microscopy. Endocrinology. 1992;130(1):364-372. doi:10.1210/endo.130.1.1727710

31. Anderson CM, Swanson RA. Astrocyte glutamate transport: review of properties, regulation, and physiological functions. Glia. 2000;32(1):1-14.

32. Diano S, Naftolin F, Horvath TL. Kainate glutamate receptors (GluR5-7) in the rat arcuate nucleus: relationship to tanycytes, astrocytes, neurons and gonadal steroid receptors. $J$ Neuroendocrinol. 1998;10(4):239-247. doi:10.1046/j.1365-2826.1998.00195.x

33. Schousboe A. Role of astrocytes in the maintenance and modulation of glutamatergic and GABAergic neurotransmission. Neurochem Res. 2003;28(2):347-352. doi:10.1023/a:1022397704922

34. Sharma G, Vijayaraghavan S. Nicotinic cholinergic signaling in hippocampal astrocytes involves calcium-induced calcium release from intracellular stores. Proc Natl Acad Sci U S A. 2001;98(7):4148-4153. doi:10.1073/pnas.071540198

35. Wang W, Ji P, Riopelle RJ, Dow KE. Functional expression of corticotropin-releasing hormone (CRH) receptor 1 in cultured rat microglia. J Neurochem. 2002;80(2):287-294. doi:10.1046/j.0022-3042.2001.00687.x

36. Han J, Kesner $P$, Metna-Laurent $M$, et al. Acute cannabinoids impair working memory through astroglial CB1 receptor modulation of hippocampal LTD. Cell. 2012;148(5):1039-1050. doi:10.1016/j.cell.2012.01.037

37. Navarrete $M$, Perea $G$, Fernandez de Sevilla $D$, et al. Astrocytes mediate in vivo cholinergicinduced synaptic plasticity. PLoS Biol. 2012;10(2):e1001259. doi:10.1371/journal.pbio.1001259

38. Halassa MM, Florian C, Fellin T, et al. Astrocytic modulation of sleep homeostasis and cognitive consequences of sleep loss. Neuron. 2009;61(2):213-219. doi:10.1016/j.neuron.2008.11.024

39. Gourine AV, Kasymov V, Marina N, et al. Astrocytes Control Breathing Through pH-Dependent Release of ATP. Science. 2010;329(5991):571-575. doi:10.1126/science.1190721

40. Sheikhbahaei S, Turovsky EA, Hosford PS, et al. Astrocytes modulate brainstem respiratory rhythm-generating circuits and determine exercise capacity. Nat Commun. 2018;9(1):370. doi:10.1038/s41467-017-02723-6

41. McDougal DH, Hermann GE, Rogers RC. Vagal Afferent Stimulation Activates Astrocytes in the Nucleus of the Solitary Tract Via AMPA Receptors: Evidence of an Atypical Neural-Glial Interaction in the Brainstem. J Neurosci. 2011;31(39):14037-14045.

doi:10.1523/JNEUROSCI.2855-11.2011 
42. Chvátal A, Benešová J, Butenko O, Verkhratsky A. Pathological potential of astroglia. :21.

43. Cabezas R, Ávila M, Gonzalez J, et al. Astrocytic modulation of blood brain barrier: perspectives on Parkinson's disease. Front Cell Neurosci. 2014;8. doi:10.3389/fncel.2014.00211

44. Liddelow SA, Barres BA. Reactive Astrocytes: Production, Function, and Therapeutic Potential. Immunity. 2017;46(6):957-967. doi:10.1016/j.immuni.2017.06.006

45. Rosciszewski G, Cadena V, Auzmendi J, et al. Detrimental Effects of HMGB-1 Require Microglial-Astroglial Interaction: Implications for the Status Epilepticus -Induced Neuroinflammation. Front Cell Neurosci. 2019;13. doi:10.3389/fncel.2019.00380

46. Verkhratsky A, Ho MS, Vardjan N, Zorec R, Parpura V. General Pathophysiology of Astroglia. Adv Exp Med Biol. 2019;1175:149-179. doi:10.1007/978-981-13-9913-8_7

47. Abg Abd Wahab DY, Gau CH, Zakaria R, et al. Review on Cross Talk between Neurotransmitters and Neuroinflammation in Striatum and Cerebellum in the Mediation of Motor Behaviour. BioMed Res Int. 2019;2019:e1767203. doi:10.1155/2019/1767203

48. Sofroniew MV, Vinters HV. Astrocytes: biology and pathology. Acta Neuropathol (Berl). 2010;119(1):7-35. doi:10.1007/s00401-009-0619-8

49. Miller SJ. Astrocyte Heterogeneity in the Adult Central Nervous System. Front Cell Neurosci. 2018;12:401. doi:10.3389/fncel.2018.00401

50. Poon DC-H, Ho Y-S, Chiu K, Wong H-L, Chang RC-C. Sickness: From the focus on cytokines, prostaglandins, and complement factors to the perspectives of neurons. Neurosci Biobehav Rev. 2015;57:30-45. doi:10.1016/j.neubiorev.2015.07.015

51. Hermann GE, Van Meter MJ, Rood JC, Rogers RC. Proteinase-Activated Receptors in the Nucleus of the Solitary Tract: Evidence for Glial-Neural Interactions in Autonomic Control of the Stomach. J Neurosci. 2009;29(29):9292-9300. doi:10.1523/JNEUROSCI.6063-08.2009

52. MacDonald AJ, Ellacott KLJ. Astrocytes in the nucleus of the solitary tract: Contributions to neural circuits controlling physiology. Physiol Behav. 2020;223.

doi:10.1016/j.physbeh.2020.112982

53. Matsui T, Omuro H, Liu Y-F, et al. Astrocytic glycogen-derived lactate fuels the brain during exhaustive exercise to maintain endurance capacity. Proc Natl Acad Sci. 2017;114(24):63586363. doi:10.1073/pnas.1702739114

54. Badimon A, Strasburger HJ, Ayata $\mathrm{P}$, et al. Negative feedback control of neuronal activity by microglia. Nature. 2020;586(7829):417-423. doi:10.1038/s41586-020-2777-8

55. Dong J, Chen X, Cui M, Yu X, Pang Q, Sun J. Beta2-Adrenergic Receptor and Astrocyte Glucose Metabolism. J Mol Neurosci. 2012;48(2):456-463. doi:10.1007/s12031-012-9742-4

56. Sriramula S, Pedersen KB, Xia H, Lazartigues E. Determining the Enzymatic Activity of Angiotensin-Converting Enzyme 2 (ACE2) in Brain Tissue and Cerebrospinal Fluid Using a Quenched Fluorescent Substrate. Methods Mol Biol Clifton NJ. 2017;1527:117-126. doi:10.1007/978-1-4939-6625-7_9 
57. Xu J, Sriramula S, Xia H, et al. Clinical Relevance and Role of Neuronal AT1 Receptors in ADAM17-Mediated ACE2 Shedding in Neurogenic Hypertension. Circ Res. 2017;121(1):43-55. doi:10.1161/CIRCRESAHA.116.310509

58. de Morais SDB, Shanks J, Zucker IH. Integrative Physiological Aspects of Brain RAS in Hypertension. Curr Hypertens Rep. 2018;20(2):10. doi:10.1007/s11906-018-0810-1

59. Sofroniew MV. Astrocyte Reactivity: Subtypes, States, and Functions in CNS Innate Immunity. Trends Immunol. 2020;41(9):758-770. doi:10.1016/j.it.2020.07.004

60. Dong Y, Benveniste EN. Immune function of astrocytes. Glia. 2001;36(2):180-190. doi:10.1002/glia.1107

61. Farina C, Aloisi F, Meinl E. Astrocytes are active players in cerebral innate immunity. Trends Immunol. 2007;28(3):138-145. doi:10.1016/j.it.2007.01.005

62. Russo M, McGavern DB. Immune surveillance of the CNS following infection and injury. Trends Immunol. 2015;36(10):637-650. doi:10.1016/j.it.2015.08.002

63. Hwang $M$, Bergmann CC. Alpha/Beta Interferon (IFN- $\alpha / \beta$ ) Signaling in Astrocytes Mediates Protection against Viral Encephalomyelitis and Regulates IFN- $\gamma$-Dependent Responses. J Virol. 2018;92(10). doi:10.1128/JVI.01901-17

64. Rosciszewski G, Cadena V, Murta V, et al. Toll-Like Receptor 4 (TLR4) and Triggering Receptor Expressed on Myeloid Cells-2 (TREM-2) Activation Balance Astrocyte Polarization into a Proinflammatory Phenotype. Mol Neurobiol. 2018;55(5):3875-3888. doi:10.1007/s12035-0170618-z

65. Rostami J, Fotaki G, Sirois J, et al. Astrocytes have the capacity to act as antigen-presenting cells in the Parkinson's disease brain. J Neuroinflammation. 2020;17(1):119.

doi:10.1186/s12974-020-01776-7

66. Skaper SD, Facci L, Giusti P. Mast cells, glia and neuroinflammation: partners in crime? Immunology. 2014;141(3):314-327. doi:10.1111/imm.12170

67. Fields RD, Woo DH, Basser PJ. Glial Regulation of the Neuronal Connectome through Local and Long-Distant Communication. Neuron. 2015;86(2):374-386. doi:10.1016/j.neuron.2015.01.014

68. Jason LA, Cotler J, Islam MF, Furst J, Sorenson M, Katz BZ. Cytokine networks analysis uncovers further differences between those who develop myalgic encephalomyelitis/chronic fatigue syndrome following infectious mononucleosis. Fatigue Biomed Health Behav. 2021;9(1):45-57. doi:10.1080/21641846.2021.1915131

69. Felger JC, Miller AH. Cytokine Effects on the Basal Ganglia and Dopamine Function: the Subcortical Source of Inflammatory Malaise. Front Neuroendocrinol. 2012;33(3):315-327. doi:10.1016/j.yfrne.2012.09.003

70. Karshikoff B, Sundelin T, Lasselin J. Role of Inflammation in Human Fatigue: Relevance of Multidimensional Assessments and Potential Neuronal Mechanisms. Front Immunol. 2017;8. doi:10.3389/fimmu.2017.00021

71. Morris G, Maes M. Myalgic encephalomyelitis/chronic fatigue syndrome and encephalomyelitis disseminata/multiple sclerosis show remarkable levels of similarity in 
phenomenology and neuroimmune characteristics. BMC Med. 2013;11(1):205. doi:10.1186/1741-7015-11-205

72. Williams MV, Cox B, Lafuse WP, Ariza ME. Epstein-Barr virus dUTPase induces neuroinflammatory mediators: Implications for Myalgic Encephalomyelitis/Chronic Fatigue Syndrome. Clin Ther. 2019;41(5):848-863. doi:10.1016/j.clinthera.2019.04.009

73. Duvignaud A, Fianu A, Bertolotti A, et al. Rheumatism and chronic fatigue, the two facets of post-chikungunya disease: the TELECHIK cohort study on Reunion island. Epidemiol Infect. 2018;146(5):633-641. doi:10.1017/S0950268818000031

74. Chiappelli F, Bakhordarian A, Thames AD, et al. Ebola: translational science considerations. J Trans/ Med. 2015;13(1):11. doi:10.1186/s12967-014-0362-3

75. Singhal $\mathrm{T}$, Cicero $\mathrm{S}, \mathrm{Pan} \mathrm{H}$, et al. Regional microglial activation in the substantia nigra is linked with fatigue in MS. Neurol - Neuroimmunol Neuroinflammation. 2020;7(5). doi:10.1212/NXI.0000000000000854

76. Komaroff A. The Biological Underpinnings of Fatigue in People with MS. Neurol Neuroimmunol Neuroinflamm. Published online August 20, 2020.

https://www.jwatch.org/na52277/2020/08/20/biological-underpinnings-fatigue-people-withms

77. Abdelhak A, Huss A, Kassubek J, Tumani H, Otto M. Serum GFAP as a biomarker for disease severity in multiple sclerosis. Sci Rep. 2018;8(1):14798. doi:10.1038/s41598-018-33158-8

78. Liu Z, Zhao F, He JJ. Hepatitis C virus (HCV) interaction with astrocytes: nonproductive infection and induction of IL-18. J Neurovirol. 2014;20(3):278-293. doi:10.1007/s13365-0140245-7

79. Yarlott $L$, Heald $E$, Forton $D$. Hepatitis $C$ virus infection, and neurological and psychiatric disorders - A review. J Adv Res. 2017;8(2):139-148. doi:10.1016/j.jare.2016.09.005

80. Werner C, Engelhard K. Pathophysiology of traumatic brain injury. BJA Br J Anaesth. 2007;99(1):4-9. doi:10.1093/bja/aem131

81. Žurek J, Fedora M. The usefulness of S100B, NSE, GFAP, NF-H, secretagogin and Hsp70 as a predictive biomarker of outcome in children with traumatic brain injury. Acta Neurochir (Wien). 2012;154(1):93-103; discussion 103. doi:10.1007/s00701-011-1175-2

82. Wylie GR, Genova H, Dobryakova E, et al. Fatigue in Gulf War Illness is associated with tonically high activation in the executive control network. Neurolmage Clin. 2019;21:101641. doi:10.1016/j.nicl.2018.101641

83. Albrecht DS, Forsberg A, Sandstrom A, et al. Brain glial activation in fibromyalgia : A multi-site positron emission tomography investigation. Brain Behav Immun. 2019;75:72-83.

84. Thapaliya K, Marshall-Gradisnik S, Staines D, Barnden L. Mapping of pathological change in chronic fatigue syndrome using the ratio of T1- and T2-weighted MRI scans. Neurolmage Clin. 2020;28:102366. doi:10.1016/j.nicl.2020.102366

85. Mizuno K, Kawatani J, Tajima K, et al. Low putamen activity associated with poor reward sensitivity in childhood chronic fatigue syndrome. Neurolmage Clin. 2016;12:600-606. doi:10.1016/j.nicl.2016.09.016 
86. Nakagawa S, Takeuchi H, Taki Y, et al. Basal ganglia correlates of fatigue in young adults. Sci Rep. 2016;6:21386. doi:10.1038/srep21386

87. Thapaliya K, Marshall-Gradisnik S, Staines D, Barnden L. Diffusion tensor imaging reveals neuronal microstructural changes in myalgic encephalomyelitis/chronic fatigue syndrome. Eur J Neurosci. 2021;54(6):6214-6228. doi:10.1111/ejn.15413

88. Bernitsas E, Yarraguntla K, Bao F, et al. Structural and Neuronal Integrity Measures of Fatigue Severity in Multiple Sclerosis. Brain Sci. 2017;7(8). doi:10.3390/brainsci7080102

89. Boissoneault J, Sevel L, Robinson ME, Staud R. Functional brain connectivity of remembered fatigue or happiness in healthy adults: Use of arterial spin labeling. J Clin Exp Neuropsychol. 2018;40(3):224-233. doi:10.1080/13803395.2017.1329407

90. Ferrero K, Silver M, Cocchetto A, Masliah E, Langford D. CNS findings in chronic fatigue syndrome and a neuropathological case report. J Investig Med Off Publ Am Fed Clin Res. 2017;65(6):974-983. doi:10.1136/jim-2016-000390

91. Goñi M, Basu N, Murray AD, Waiter GD. Neural Indicators of Fatigue in Chronic Diseases: A Systematic Review of MRI Studies. Diagnostics. 2018;8(3). doi:10.3390/diagnostics8030042

92. Hou L, Song Z, Pan ZJ, Cheng JL, Yu Y, Wang J. Decreased Activation of Subcortical Brain Areas in the Motor Fatigue State: An fMRI Study. Front Psychol. 2016;7.

doi:10.3389/fpsyg.2016.01154

93. MacHale SM, Lawŕie SM, Cavanagh JT, et al. Cerebral perfusion in chronic fatigue syndrome and depression. Br J Psychiatry J Ment Sci. 2000;176:550-556. doi:10.1192/bjp.176.6.550

94. Chaudhuri A, Behan PO. Fatigue in neurological disorders. Lancet Lond Engl. 2004;363(9413):978-988. doi:10.1016/S0140-6736(04)15794-2

95. Barnden LR, Crouch B, Kwiatek R, et al. A brain MRI study of chronic fatigue syndrome: evidence of brainstem dysfunction and altered homeostasis. NMR Biomed. 2011;24(10):13021312. doi:10.1002/nbm.1692

96. Dickinson CJ. Chronic fatigue syndrome-aetiological aspects. Eur J Clin Invest. 1997;27(4):257-267. doi:10.1046/j.1365-2362.1997.1120664.x

97. Staub F, Bogousslavsky J. Fatigue after stroke: a major but neglected issue. Cerebrovasc Dis Basel Switz. 2001;12(2):75-81. doi:10.1159/000047685

98. Derevenco P, Stoica N, Sovrea I, Imreh S. Central and peripheral effects of 6-hydroxydopamine on exercise performance in rats. Psychoneuroendocrinology. 1986;11(2):141-153.

doi:10.1016/0306-4530(86)90049-1

99. Boev VM, Krauz VA. [Functional state of the hippocampo-reticular complex during submaximal physical loading and fatigue]. Zh Vyssh Nerv Deiat Im I P Pavlova. 1981;31(5):1029-1037.

100. Noda M, Ifuku M, Hossain MdS, Katafuchi T. Glial Activation and Expression of the Serotonin Transporter in Chronic Fatigue Syndrome. Front Psychiatry. 2018;9. doi:10.3389/fpsyt.2018.00589 
101. Nakatomi Y, Mizuno K, Ishii A, et al. Neuroinflammation in Patients with Chronic Fatigue Syndrome/Myalgic Encephalomyelitis: An ${ }^{11} \mathrm{C}-(\mathrm{R})$-PK11195 PET Study. J Nucl Med Off Publ Soc Nucl Med. 2014;55(6):945-950. doi:10.2967/jnumed.113.131045

102. Morriss RK, Wearden AJ, Battersby L. The relation of sleep difficulties to fatigue, mood and disability in chronic fatigue syndrome. J Psychosom Res. 1997;42(6):597-605. doi:10.1016/s0022-3999(97)89895-9

103. Pedersen M, Ekstedt M, Småstuen MC, et al. Sleep-wake rhythm disturbances and perceived sleep in adolescent chronic fatigue syndrome. J Sleep Res. 2017;26(5):595-601. doi:10.1111/jsr.12547

104. Cambras T, Castro-Marrero J, Zaragoza MC, Díez-Noguera A, Alegre J. Circadian rhythm abnormalities and autonomic dysfunction in patients with Chronic Fatigue Syndrome/Myalgic Encephalomyelitis. PLOS ONE. 2018;13(6). doi:10.1371/journal.pone.0198106

105. Buijs RM, la Fleur SE, Wortel J, et al. The suprachiasmatic nucleus balances sympathetic and parasympathetic output to peripheral organs through separate preautonomic neurons. $J$ Comp Neurol. 2003;464(1):36-48. doi:10.1002/cne.10765

106. Benca R, Duncan MJ, Frank E, McClung C, Nelson RJ, Vicentic A. Biological rhythms, higher brain function, and behavior: Gaps, opportunities, and challenges. Brain Res Rev. 2009;62(1):57-70. doi:10.1016/j.brainresrev.2009.09.005

107. Ishibashi T, Dakin KA, Stevens B, et al. Astrocytes Promote Myelination in Response to Electrical Impulses. Neuron. 2006;49(6):823-832. doi:10.1016/j.neuron.2006.02.006

108. N P, Mj L, Sa P, Da G. Paying the circadian toll: the circadian response to LPS injection is dependent on the Toll-like receptor 4. J Neuroimmunol. 2010;225(1-2):62-67. doi:10.1016/j.jneuroim.2010.04.015

109. Bentivoglio PM, Deng X-H, Nygård M, Sadki A, Kristensson K. The aging suprachiasmatic nucleus and cytokines: functional, molecular, and cellular changes in rodents. Chronobiol Int. 2006;23(1-2):437-449. doi:10.1080/07420520500545797

110. Shan ZY, Barnden LR, Kwiatek RA, Bhuta S, Hermens DF, Lagopoulos J. Neuroimaging characteristics of myalgic encephalomyelitis/chronic fatigue syndrome (ME/CFS): a systematic review. J Transl Med. 2020;18(1):335. doi:10.1186/s12967-020-02506-6

111. Barnden LR, Crouch B, Kwiatek R, Burnet R, Del Fante P. Evidence in chronic fatigue syndrome for severity-dependent upregulation of prefrontal myelination that is independent of anxiety and depression. NMR Biomed. 2015;28(3):404-413. doi:10.1002/nbm.3261

112. Gibbs ME, Hutchinson D, Hertz L. Astrocytic involvement in learning and memory consolidation. Neurosci Biobehav Rev. 2008;32(5):927-944. doi:10.1016/j.neubiorev.2008.02.001

113. Santello $\mathrm{M}$, Toni $\mathrm{N}$, Volterra $\mathrm{A}$. Astrocyte function from information processing to cognition and cognitive impairment. Nat Neurosci. 2019;22(2):154-166. doi:10.1038/s41593-018-0325-8

114. Gao V, Suzuki A, Magistretti PJ, et al. Astrocytic $\beta 2$-adrenergic receptors mediate hippocampal long-term memory consolidation. Proc Natl Acad Sci. 2016;113(30):8526-8531. 
115. O'Dell TJ, Connor SA, Guglietta R, Nguyen PV. $\beta$-Adrenergic receptor signaling and modulation of long-term potentiation in the mammalian hippocampus. Learn Mem. 2015;22(9):461-471. doi:10.1101/Im.031088.113

116. Finsterwald C, Magistretti PJ, Lengacher S. Astrocytes: New Targets for the Treatment of Neurodegenerative Diseases. Curr Pharm Des. 2015;21(25):3570-3581. doi:10.2174/1381612821666150710144502

117. Hansson E, Rönnbäck L. Altered neuronal-glial signaling in glutamatergic transmission as a unifying mechanism in chronic pain and mental fatigue. Neurochem Res. 2004;29(5):989-996. doi:10.1023/b:nere.0000021243.86287.43

118. Rönnbäck L, Hansson E. On the potential role of glutamate transport in mental fatigue. $J$ Neuroinflammation. 2004;1:22. doi:10.1186/1742-2094-1-22

119. Gandevia SC. Spinal and supraspinal factors in human muscle fatigue. Physiol Rev. 2001;81(4):1725-1789. doi:10.1152/physrev.2001.81.4.1725

120. Bigland-Ritchie B, Jones DA, Hosking GP, Edwards RH. Central and peripheral fatigue in sustained maximum voluntary contractions of human quadriceps muscle. Clin Sci Mol Med. 1978;54(6):609-614. doi:10.1042/cs0540609

121. Wan J, Qin Z, Wang P, Sun Y, Liu X. Muscle fatigue: general understanding and treatment. Exp Mol Med. 2017;49(10):e384-e384. doi:10.1038/emm.2017.194

122. MI S, Js K, Sp van der W, Bg van E, G B, Mj Z. Diminished central activation during maximal voluntary contraction in chronic fatigue syndrome. Clin Neurophysiol Off J Int Fed Clin Neurophysiol. 2004;115(11):2518-2524. doi:10.1016/j.clinph.2004.06.007

123. Siemionow V, Fang Y, Calabrese L, Sahgal V, Yue GH. Altered central nervous system signal during motor performance in chronic fatigue syndrome. Clin Neurophysiol Off J Int Fed Clin Neurophysiol. 2004;115(10):2372-2381. doi:10.1016/j.clinph.2004.05.012

124. Kent-Braun JA, Sharma KR, Weiner MW, Massie B, Miller RG. Central basis of muscle fatigue in chronic fatigue syndrome. Neurology. 1993;43(1):125-131. doi:10.1212/wnl.43.1_part_1.125

125. Chaudhuri A, Behan PO. Fatigue and basal ganglia. J Neurol Sci. 2000;179(S 1-2):34-42. doi:10.1016/s0022-510x(00)00411-1

126. Weissenborn K, Ennen JC, Bokemeyer M, et al. Monoaminergic neurotransmission is altered in hepatitis $\mathrm{C}$ virus infected patients with chronic fatigue and cognitive impairment. Gut. 2006;55(11):1624-1630. doi:10.1136/gut.2005.080267

127. Felger JC, Treadway MT. Inflammation Effects on Motivation and Motor Activity: Role of Dopamine. Neuropsychopharmacology. 2017;42(1):216-241. doi:10.1038/npp.2016.143

128. Erta M, Giralt M, Esposito FL, Fernandez-Gayol O, Hidalgo J. Astrocytic IL-6 mediates locomotor activity, exploration, anxiety, learning and social behavior. Horm Behav. 2015;73:64-74. doi:10.1016/j.yhbeh.2015.06.016

129. Corkrum M, Covelo A, Lines J, et al. Dopamine-Evoked Synaptic Regulation in the Nucleus Accumbens Requires Astrocyte Activity. Neuron. 2020;105(6):1036-1047.e5. doi:10.1016/j.neuron.2019.12.026 
130. Zhou X, Xiao Q, Xie L, Yang F, Wang L, Tu J. Astrocyte, a Promising Target for Mood Disorder Interventions. Front Mol Neurosci. 2019;12. doi:10.3389/fnmol.2019.00136

131. Mayegowda SB, Thomas C. Glial pathology in neuropsychiatric disorders: a brief review. J Basic Clin Physiol Pharmacol. 2019;30(4). doi:10.1515/jbcpp-2018-0120

132. Miguel-Hidalgo JJ. The role of glial cells in drug abuse. Curr Drug Abuse Rev. 2009;2(1):72-82.

133. Gomborone JE, Gorard DA, Dewsnap PA, Libby GW, Farthing MJ. Prevalence of irritable bowel syndrome in chronic fatigue. J R Coll Physicians Lond. 1996;30(6):512-513.

134. Sheedy JR, Wettenhall REH, Scanlon $D$, et al. Increased d-lactic Acid intestinal bacteria in patients with chronic fatigue syndrome. Vivo Athens Greece. 2009;23(4):621-628.

135. Frémont M, Coomans D, Massart S, De Meirleir K. High-throughput $16 \mathrm{~S}$ rRNA gene sequencing reveals alterations of intestinal microbiota in myalgic encephalomyelitis/chronic fatigue syndrome patients. Anaerobe. 2013;22:50-56. doi:10.1016/j.anaerobe.2013.06.002

136. Maes M, Mihaylova I, Leunis J-C. Increased serum IgA and IgM against LPS of enterobacteria in chronic fatigue syndrome (CFS): Indication for the involvement of gram-negative enterobacteria in the etiology of CFS and for the presence of an increased gut-intestinal permeability. J Affect Disord. 2007;99(1):237-240. doi:10.1016/j.jad.2006.08.021

137. Giloteaux L, Goodrich JK, Walters WA, Levine SM, Ley RE, Hanson MR. Reduced diversity and altered composition of the gut microbiome in individuals with myalgic encephalomyelitis/chronic fatigue syndrome. Microbiome. 2016;4(1):30. doi:10.1186/s40168016-0171-4

138. Newberry F, Hsieh S-Y, Wileman T, Carding SR. Does the microbiome and virome contribute to myalgic encephalomyelitis/chronic fatigue syndrome? Clin Sci. 2018;132(5):523-542. doi:10.1042/CS20171330

139. Komaroff AL, Lipkin WI. Insights from Myalgic Encephalomyelitis/Chronic Fatigue Syndrome May Help Unravel the Pathogenesis of Post-Acute COVID-19 Syndrome. Trends Mol Med. Published online June 7, 2021. doi:10.1016/j.molmed.2021.06.002

140. Proal A, Marshall T. Myalgic Encephalomyelitis/Chronic Fatigue Syndrome in the Era of the Human Microbiome: Persistent Pathogens Drive Chronic Symptoms by Interfering With Host Metabolism, Gene Expression, and Immunity. Front Pediatr. 2018;6. doi:10.3389/fped.2018.00373

141. Giovannoni F, Quintana FJ. The Role of Astrocytes in CNS Inflammation. Trends Immunol. 2020;41(9):805-819. doi:10.1016/j.it.2020.07.007

142. Burnet $\mathrm{RB}, \mathrm{Chatterton} \mathrm{BE}$. Gastric emptying is slow in chronic fatigue syndrome. $B M C$ Gastroenterol. 2004;4(1):32. doi:10.1186/1471-230X-4-32

143. Stussman B, Williams A, Snow J, et al. Characterization of Post-exertional Malaise in Patients With Myalgic Encephalomyelitis/Chronic Fatigue Syndrome. Front Neurol. 2020;11. doi:10.3389/fneur.2020.01025

144. Shukla SK, Cook D, Meyer J, et al. Changes in Gut and Plasma Microbiome following Exercise Challenge in Myalgic Encephalomyelitis/Chronic Fatigue Syndrome (ME/CFS). PloS One. 2015;10(12):e0145453. doi:10.1371/journal.pone.0145453 
145. Diniz LP, Tortelli V, Matias I, et al. Astrocyte Transforming Growth Factor Beta 1 Protects Synapses against A $\beta$ Oligomers in Alzheimer's Disease Model. J Neurosci. 2017;37(28):67976809. doi:10.1523/JNEUROSCI.3351-16.2017

146. Diniz LP, Matias I, Araujo APB, et al. $\alpha$-synuclein oligomers enhance astrocyte-induced synapse formation through TGF- $\beta 1$ signaling in a Parkinson's disease model. J Neurochem. 2019;150(2):138-157. doi:10.1111/jnc.14710

147. Matias I, Morgado J, Gomes FCA. Astrocyte Heterogeneity: Impact to Brain Aging and Disease. Front Aging Neurosci. 2019;11. doi:10.3389/fnagi.2019.00059

148. Colombo E, Farina C. Astrocytes: Key Regulators of Neuroinflammation. Trends Immunol. 2016;37(9):608-620. doi:10.1016/j.it.2016.06.006

149. Murta V, Villarreal A, Ramos AJ. Severe Acute Respiratory Syndrome Coronavirus 2 Impact on the Central Nervous System: Are Astrocytes and Microglia Main Players or Merely Bystanders? ASN Neuro. 2020;12:1759091420954960. doi:10.1177/1759091420954960

150. Salter MW, Beggs S. Sublime Microglia: Expanding Roles for the Guardians of the CNS. Cell. 2014;158(1):15-24. doi:10.1016/j.cell.2014.06.008

151. Tay TL, Savage JC, Hui CW, Bisht K, Tremblay M-È. Microglia across the lifespan: from origin to function in brain development, plasticity and cognition. J Physiol. 2017;595(6):1929-1945. doi:10.1113/JP272134

152. Cooper DM, Radom-Aizik S, Schwindt C, Zaldivar F. Dangerous exercise: lessons learned from dysregulated inflammatory responses to physical activity. J Appl Physiol. 2007;103(2). Accessed August 20, 2021. https://cyberleninka.org/article/n/1496473

153. Gaab J, Rohleder N, Heitz V, et al. Stress-induced changes in LPS-induced pro-inflammatory cytokine production in chronic fatigue syndrome. Psychoneuroendocrinology. 2005;30(2):188198. doi:10.1016/j.psyneuen.2004.06.008

154. Light AR, White AT, Hughen RW, Light KC. Moderate exercise increases expression for sensory, adrenergic, and immune genes in chronic fatigue syndrome patients but not in normal subjects. J Pain. 2009;10(10):1099-1112. doi:10.1016/j.jpain.2009.06.003

155. White AT, Light AR, Hughen RW, et al. Severity of symptom flare after moderate exercise is linked to cytokine activity in chronic fatigue syndrome. Psychophysiology. 2010;47(4):615-624. doi:10.1111/j.1469-8986.2010.00978.x

156. Jammes Y, Steinberg JG, Mambrini O, Brégeon F, Delliaux S. Chronic fatigue syndrome: assessment of increased oxidative stress and altered muscle excitability in response to incremental exercise. J Intern Med. 2005;257(3):299-310. doi:10.1111/j.13652796.2005.01452.x

157. Sorensen B, Streib JE, Strand M, et al. Complement activation in a model of chronic fatigue syndrome. J Allergy Clin Immunol. 2003;112(2):397-403. doi:10.1067/mai.2003.1615

158. Sorensen B, Jones JF, Vernon SD, Rajeevan MS. Transcriptional control of complement activation in an exercise model of chronic fatigue syndrome. Mol Med Camb Mass. 2009;15(12):34-42. doi:10.2119/molmed.2008.00098 
159. Nijs J, Nees A, Paul L, et al. Altered immune response to exercise in patients with chronic fatigue syndrome/myalgic encephalomyelitis: a systematic literature review. Exerc Immunol Rev. 2014;20:94-116.

160. Biancardi VC, Stranahan AM, Krause EG, de Kloet AD, Stern JE. Cross talk between AT1 receptors and Toll-like receptor 4 in microglia contributes to angiotensin II-derived ROS production in the hypothalamic paraventricular nucleus. Am J Physiol-Heart Circ Physiol. 2016;310(3):H404-H415. doi:10.1152/ajpheart.00247.2015

161. Rietdijk CD, Wezel RJA van, Garssen J, Kraneveld AD. Neuronal toll-like receptors and neuroimmunity in Parkinson's disease, Alzheimer's disease and stroke. Neuroimmunol Neuroinflammation. 2016;3:27-37. doi:10.20517/2347-8659.2015.28

162. Moneghetti KJ, Skhiri M, Contrepois K, et al. Value of Circulating Cytokine Profiling During Submaximal Exercise Testing in Myalgic Encephalomyelitis/Chronic Fatigue Syndrome. Sci Rep. 2018;8. doi:10.1038/s41598-018-20941-w

163. Michlmayr D, McKimmie CS. Role of CXCL10 in central nervous system inflammation. Int J Interferon Cytokine Mediat Res. 2014;6:1-18. doi:10.2147/IJICMR.S35953

164. Theoharides TC, Tsilioni I, Natelson B. Exosome-associated Mitochondrial DNA is Elevated in Patients with ME/CFS and Stimulates Human Cultured Microglia to Secrete IL-1ß. Published online September 19, 2021. doi:10.21203/rs.3.rs-154011/v1

165. Cook DB, Light AR, Light KC, et al. Neural consequences of post-exertion malaise in Myalgic Encephalomyelitis/Chronic Fatigue Syndrome. Brain Behav Immun. 2017;62:87-99. doi:10.1016/j.bbi.2017.02.009

166. Washington SD, Rayhan RU, Garner R, et al. Exercise alters brain activation in Gulf War Illness and Myalgic Encephalomyelitis/Chronic Fatigue Syndrome. Brain Commun. 2020;2(2). doi:10.1093/braincomms/fcaa070

167. Ramírez-Barrantes $\mathrm{R}$, Cordova $\mathrm{C}$, Poblete $\mathrm{H}$, et al. Perspectives of TRPV1 Function on the Neurogenesis and Neural Plasticity. Neural Plast. 2016;2016:1568145. doi:10.1155/2016/1568145

168. Henry RJ, Kerr DM, Flannery LE, et al. Pharmacological inhibition of FAAH modulates TLRinduced neuroinflammation, but not sickness behaviour: An effect partially mediated by central TRPV1. Brain Behav Immun. 2017;62:318-331. doi:10.1016/j.bbi.2017.02.016

169. Idzko M, Ferrari D, Eltzschig HK. Nucleotide signalling during inflammation. Nature. 2014;509(7500):310-317. doi:10.1038/nature13085

170. Low RN, Low RJ, Akrami A. A Cytokine-based model for the pathophysiology of Long COVID symptoms. Published online November 12, 2020. doi:10.31219/osf.io/7gcnv

171. Cerqueira É, Marinho DA, Neiva HP, Lourenço O. Inflammatory Effects of High and Moderate Intensity Exercise-A Systematic Review. Front Physiol. 2020;10. doi:10.3389/fphys.2019.01550

172. Keller C, Steensberg A, Hansen AK, Fischer CP, Plomgaard P, Pedersen BK. Effect of exercise, training, and glycogen availability on IL-6 receptor expression in human skeletal muscle. J AppI Physiol. 2005;99(6):2075-2079. doi:10.1152/japplphysiol.00590.2005 
173. K S, K S. Characterization of Exercise-Induced Cytokine Release, the Impacts on the Body, the Mechanisms and Modulations. doi:10.23937/2469-5718/1510122

174. Fischer CP. Interleukin- 6 in acute exercise and training: what is the biological relevance? Exerc Immunol Rev. 2006;12:6-33.

175. Fluge $\varnothing$, Mella $O$, Bruland $O$, et al. Metabolic profiling indicates impaired pyruvate dehydrogenase function in myalgic encephalopathy/chronic fatigue syndrome. JCl Insight. 2017;1(21). doi:10.1172/jci.insight.89376

176. Marsland AL, Walsh C, Lockwood K, John-Henderson NA. The effects of acute psychological stress on circulating and stimulated inflammatory markers: A systematic review and metaanalysis. Brain Behav Immun. 2017;64:208-219. doi:10.1016/j.bbi.2017.01.011

177. Bateman RJ, Boychuk CR, Philbin KE, Mendelowitz D. $\beta$ adrenergic receptor modulation of neurotransmission to cardiac vagal neurons in the nucleus ambiguus. Neuroscience. 2012;210:58-66. doi:10.1016/j.neuroscience.2012.02.033

178. Kolmus K, Tavernier J, Gerlo S. $\beta 2$-Adrenergic receptors in immunity and inflammation: stressing NF-KB. Brain Behav Immun. 2015;45:297-310. doi:10.1016/j.bbi.2014.10.007

179. Wu L, Tai Y, Hu S, et al. Bidirectional Role of $\beta 2$-Adrenergic Receptor in Autoimmune Diseases. Front Pharmacol. 2018;9. doi:10.3389/fphar.2018.01313

180. Vida G, Peña G, Kanashiro A, et al. $\beta 2$-Adrenoreceptors of regulatory lymphocytes are essential for vagal neuromodulation of the innate immune system. FASEB J. 2011;25(12):44764485. doi:10.1096/fj.11-191007

181. Sharma M, Flood PM. Adrenergic Receptors as Pharmacological Targets for Neuroinflammation and Neurodegeneration in Parkinson's Disease. IntechOpen; 2018. doi:10.5772/intechopen.81343

182. Junker V, Becker A, Hühne R, et al. Stimulation of beta-adrenoceptors activates astrocytes and provides neuroprotection. Eur J Pharmacol. 2002;446(1-3):25-36. doi:10.1016/s00142999(02)01814-9

183. Zhang $X$, Hartung JE, Bortsov AV, et al. Sustained stimulation of $\beta 2$ - and $\beta 3$-adrenergic receptors leads to persistent functional pain and neuroinflammation. Brain Behav Immun. 2018;73:520-532. doi:10.1016/j.bbi.2018.06.017

184. DiSabato DJ, Quan N, Godbout JP. Neuroinflammation: the devil is in the details. J Neurochem. 2016;139(S2):136-153. doi:10.1111/jnc.13607

185. Lyman M, Lloyd DG, Ji X, Vizcaychipi MP, Ma D. Neuroinflammation: the role and consequences. Neurosci Res. 2014;79:1-12. doi:10.1016/j.neures.2013.10.004

186. Stasiłowicz A, Tykarska E, Rosiak N, et al. $<\mathrm{p}>$ The Inclusion of Tolfenamic Acid into Cyclodextrins Stimulated by Microenvironmental pH Modification as a Way to Increase the Anti-Migraine Effect</p>. J Pain Res. 2021;14:981-992. doi:10.2147/JPR.S295795

187. Kharraziha I, Axelsson J, Ricci F, et al. Serum Activity Against G Protein-Coupled Receptors and Severity of Orthostatic Symptoms in Postural Orthostatic Tachycardia Syndrome. J Am Heart Assoc. 2020;9(15):e015989. doi:10.1161/JAHA.120.015989 
188. Kohr $D$, Singh $P$, Tschernatsch $M$, et al. Autoimmunity against the $\beta 2$ adrenergic receptor and muscarinic-2 receptor in complex regional pain syndrome. Pain. 2011;152(12):2690-2700. doi:10.1016/j.pain.2011.06.012

189. Licastro F, Pedrini S, Caputo L, et al. Increased plasma levels of interleukin-1, interleukin- 6 and $\alpha$-1-antichymotrypsin in patients with Alzheimer's disease: peripheral inflammation or signals from the brain? J Neuroimmunol. 2000;103(1):97-102. doi:10.1016/S0165-5728(99)00226-X

190. VanElzakker MB, Brumfield SA, Lara Mejia PS. Neuroinflammation and Cytokines in Myalgic Encephalomyelitis/Chronic Fatigue Syndrome (ME/CFS): A Critical Review of Research Methods. Front Neurol. 2019;9. doi:10.3389/fneur.2018.01033

191. Kerr JR. Epstein-Barr Virus Induced Gene-2 Upregulation Identifies a Particular Subtype of Chronic Fatigue Syndrome/Myalgic Encephalomyelitis. Front Pediatr. 2019;7:59. doi:10.3389/fped.2019.00059

192. Rutkowska A, Shimshek DR, Sailer AW, Dev KK. EBI2 regulates pro-inflammatory signalling and cytokine release in astrocytes. Neuropharmacology. 2018;133:121-128. doi:10.1016/j.neuropharm.2018.01.029

193. Glassford JAG. The Neuroinflammatory Etiopathology of Myalgic Encephalomyelitis/Chronic Fatigue Syndrome (ME/CFS). Front Physiol. 2017;8. doi:10.3389/fphys.2017.00088

194. Qin L, Wu X, Block ML, et al. Systemic LPS causes chronic neuroinflammation and progressive neurodegeneration. Glia. 2007;55(5):453-462. doi:10.1002/glia.20467

195. Hornig M, Gottschalk CG, Eddy ML, et al. Immune network analysis of cerebrospinal fluid in myalgic encephalomyelitis/chronic fatigue syndrome with atypical and classical presentations. Transl Psychiatry. 2017;7(4):e1080. doi:10.1038/tp.2017.44

196. Natelson BH, Weaver SA, Tseng C-L, Ottenweller JE. Spinal Fluid Abnormalities in Patients with Chronic Fatigue Syndrome. Clin Diagn Lab Immunol. 2005;12(1):52-55.

doi:10.1128/CDLI.12.1.52-55.2005

197. Peterson D, Brenu EW, Gottschalk G, et al. Cytokines in the Cerebrospinal Fluids of Patients with Chronic Fatigue Syndrome/Myalgic Encephalomyelitis. Mediators Inflamm. 2015;2015. doi:10.1155/2015/929720

198. Abou-Donia MB, Lapadula ES, Krengel MH, et al. Using Plasma Autoantibodies of Central Nervous System Proteins to Distinguish Veterans with Gulf War Illness from Healthy and Symptomatic Controls. Brain Sci. 2020;10(9). doi:10.3390/brainsci10090610

199. Castro-Marrero J, Zacares M, Almenar-Pérez E, Alegre-Martín J, Oltra E. Complement Component C1q as a Potential Diagnostic Tool for Myalgic Encephalomyelitis/Chronic Fatigue Syndrome Subtyping. J Clin Med. 2021;10(18):4171. doi:10.3390/jcm10184171

200. Fonseca MI, Chu S-H, Hernandez MX, et al. Cell-specific deletion of C1qa identifies microglia as the dominant source of C1q in mouse brain. J Neuroinflammation. 2017;14(1):48. doi:10.1186/s12974-017-0814-9

201. Schäfer MK-H, Schwaeble WJ, Post C, et al. Complement C1q Is Dramatically Up-Regulated in Brain Microglia in Response to Transient Global Cerebral Ischemia. J Immunol. 2000;164(10):5446-5452. doi:10.4049/jimmunol.164.10.5446 
202. Molecular Aspects of Neurodegeneration and Neuroprotection. https://www.eurekaselect.com. Accessed September 22, 2021. https://www.eurekaselect.com/52607/volume/1

203. Puri BK, Counsell SJ, Zaman R, et al. Relative increase in choline in the occipital cortex in chronic fatigue syndrome. Acta Psychiatr Scand. 2002;106(3):224-226. doi:10.1034/j.16000447.2002.01300.x

204. Tomoda A, Miike T, Yamada E, et al. Chronic fatigue syndrome in childhood. Brain Dev. 2000;22(1):60-64. doi:10.1016/s0387-7604(99)00111-4

205. Chaudhuri A, Condon BR, Gow JW, Brennan D, Hadley DM. Proton magnetic resonance spectroscopy of basal ganglia in chronic fatigue syndrome. Neuroreport. 2003;14(2):225-228. doi:10.1097/00001756-200302100-00013

206. Godlewska BR, Williams S, Emir UE, et al. Neurochemical abnormalities in chronic fatigue syndrome: a pilot magnetic resonance spectroscopy study at 7 Tesla. Psychopharmacology (Berl). Published online October 5, 2021:1-9. doi:10.1007/s00213-021-05986-6

207. Mathew SJ, Mao X, Keegan KA, et al. Ventricular cerebrospinal fluid lactate is increased in chronic fatigue syndrome compared with generalized anxiety disorder: an in vivo $3.0 \mathrm{~T} \mathrm{(1) \textrm {H }}$ MRS imaging study. NMR Biomed. 2009;22(3):251-258. doi:10.1002/nbm.1315

208. Murrough JW, Mao X, Collins KA, et al. Increased ventricular lactate in chronic fatigue syndrome measured by $1 \mathrm{H}$ MRS imaging at 3.0 T. II: comparison with major depressive disorder. NMR Biomed. 2010;23(6):643-650. doi:10.1002/nbm.1512

209. Natelson BH, Mao X, Stegner AJ, et al. Multimodal and Simultaneous Assessments of Brain and Spinal Fluid Abnormalities in Chronic Fatigue Syndrome and the Effects of Psychiatric Comorbidty. J Neurol Sci. 2017;375:411-416. doi:10.1016/j.jns.2017.02.046

210. Natelson BH, Vu D, Coplan JD, et al. Elevations of Ventricular Lactate Levels Occur in Both Chronic Fatigue Syndrome and Fibromyalgia. Fatigue Biomed Health Behav. 2017;5(1):15-20. doi:10.1080/21641846.2017.1280114

211. Shungu DC, Weiduschat N, Murrough JW, et al. Increased ventricular lactate in chronic fatigue syndrome. III. Relationships to cortical glutathione and clinical symptoms implicate oxidative stress in disorder pathophysiology. NMR Biomed. 2012;25(9):1073-1087. doi:10.1002/nbm.2772

212. Shan ZY, Finegan K, Bhuta S, et al. Decreased Connectivity and Increased Blood Oxygenation Level Dependent Complexity in the Default Mode Network in Individuals with Chronic Fatigue Syndrome. Brain Connect. 2018;8(1):33-39. doi:10.1089/brain.2017.0549

213. Cw G, Me R, S L, et al. Abnormal Resting-State Functional Connectivity in Patients with Chronic Fatigue Syndrome: Results of Seed and Data-Driven Analyses. Brain Connect. 2015;6(1):48-56. doi:10.1089/brain.2015.0366

214. Zhang Z-T, Du X-M, Ma X-J, et al. Activation of the NLRP3 inflammasome in lipopolysaccharideinduced mouse fatigue and its relevance to chronic fatigue syndrome. J Neuroinflammation. 2016;13. doi:10.1186/s12974-016-0539-1 
215. O'Brien WT, Pham L, Symons GF, Monif M, Shultz SR, McDonald SJ. The NLRP3 inflammasome in traumatic brain injury: potential as a biomarker and therapeutic target. $J$

Neuroinflammation. 2020;17. doi:10.1186/s12974-020-01778-5

216. Anders H-J, Schaefer L. Beyond tissue injury-damage-associated molecular patterns, toll-like receptors, and inflammasomes also drive regeneration and fibrosis. J Am Soc Nephrol JASN. 2014;25(7):1387-1400. doi:10.1681/ASN.2014010117

217. Singhal G, Jaehne EJ, Corrigan F, Toben C, Baune BT. Inflammasomes in neuroinflammation and changes in brain function: a focused review. Front Neurosci. 2014;8. doi:10.3389/fnins.2014.00315

218. Ceyzériat K, Abjean L, Carrillo-de Sauvage M-A, Ben Haim L, Escartin C. The complex STATes of astrocyte reactivity: How are they controlled by the JAK-STAT3 pathway? Neuroscience. 2016;330:205-218. doi:10.1016/j.neuroscience.2016.05.043

219. Cheema AK, Sarria L, Bekheit M, et al. Unravelling myalgic encephalomyelitis/chronic fatigue syndrome (ME/CFS): Gender-specific changes in the microRNA expression profiling in ME/CFS. J Cell Mol Med. 2020;24(10):5865-5877. doi:10.1111/jcmm.15260

220. Tang Y, Dong X, Chen G, et al. Vagus Nerve Stimulation Attenuates Early Traumatic Brain Injury by Regulating the NF-KB/NLRP3 Signaling Pathway. Neurorehabil Neural Repair. 2020;34(9):831-843. doi:10.1177/1545968320948065

221. Beaumont A, Burton AR, Lemon J, Bennett BK, Lloyd A, Vollmer-Conna U. Reduced Cardiac Vagal Modulation Impacts on Cognitive Performance in Chronic Fatigue Syndrome. PLOS ONE. 2012;7(11). doi:10.1371/journal.pone.0049518

222. Wright JW, Harding JW. The brain renin-angiotensin system: a diversity of functions and implications for CNS diseases. Pflüg Arch - Eur J Physiol. 2013;465(1):133-151. doi:10.1007/s00424-012-1102-2

223. Vital SA, Terao S, Nagai M, Granger DN. Mechanisms underlying the cerebral microvascular responses to angiotensin II-induced hypertension. Microcirc N Y N 1994. 2010;17(8):641. doi:10.1111/j.1549-8719.2010.00060.x

224. Mustafa HI, Garland EM, Biaggioni I, et al. Abnormalities of Angiotensin Regulation in Postural Tachycardia Syndrome. Heart Rhythm Off J Heart Rhythm Soc. 2011;8(3):422. doi:10.1016/j.hrthm.2010.11.009

225. Ferguson AV, Washburn DLS, Latchford KJ. Hormonal and Neurotransmitter Roles for Angiotensin in the Regulation of Central Autonomic Function. Exp Biol Med. 2001;226(2):8596. doi:10.1177/153537020122600205

226. Tsuda K. Renin-Angiotensin System and Sympathetic Neurotransmitter Release in the Central Nervous System of Hypertension. Int J Hypertens. 2012;2012. doi:10.1155/2012/474870

227. Miller AJ, Arnold AC. The Renin-Angiotensin System in Cardiovascular Autonomic Control: Recent Developments and Clinical Implications. Clin Auton Res Off J Clin Auton Res Soc. 2019;29(2):231-243. doi:10.1007/s10286-018-0572-5

228. de Kloet AD, Liu M, Rodríguez V, Krause EG, Sumners C. Role of neurons and glia in the CNS actions of the renin-angiotensin system in cardiovascular control. Am J Physiol - Regul Integr Comp Physiol. 2015;309(5):R444-R458. doi:10.1152/ajpregu.00078.2015 
229. Naffah-Mazzacoratti M da G, Gouveia TLF, Simões PSR, Perosa SR. What have we learned about the kallikrein-kinin and renin-angiotensin systems in neurological disorders? World J Biol Chem. 2014;5(2):130-140. doi:10.4331/wjbc.v5.i2.130

230. Kasparov S, Paton JF. Differential effects of angiotensin II in the nucleus tractus solitarii of the rat--plausible neuronal mechanism. J Physiol. 1999;521 Pt 1:227-238. doi:10.1111/j.14697793.1999.00227.x

231. Gao L, Schultz HD, Patel KP, Zucker IH, Wang W. Augmented Input From Cardiac Sympathetic Afferents Inhibits Baroreflex in Rats With Heart Failure. Hypertension. 2005;45(6):1173-1181. doi:10.1161/01.HYP.0000168056.66981.c2

232. Cosarderelioglu C, Nidadavolu LS, George CJ, et al. Brain Renin-Angiotensin System at the Intersect of Physical and Cognitive Frailty. Front Neurosci. 2020;14. doi:10.3389/fnins.2020.586314

233. Nakagawa P, Gomez J, Grobe JL, Sigmund CD. The Renin-Angiotensin System in the Central Nervous System and Its Role in Blood Pressure Regulation. Curr Hypertens Rep. 2020;22(1):7. doi:10.1007/s11906-019-1011-2

234. Bodiga VL, Bodiga S. Renin Angiotensin System in Cognitive Function and Dementia. Asian J Neurosci. 2013;2013:e102602. doi:10.1155/2013/102602

235. Labandeira-Garcia JL, Rodríguez-Perez Al, Garrido-Gil P, Rodriguez-Pallares J, Lanciego JL, Guerra MJ. Brain Renin-Angiotensin System and Microglial Polarization: Implications for Aging and Neurodegeneration. Front Aging Neurosci. 2017;9. doi:10.3389/fnagi.2017.00129

236. Wallukat G, Hohberger B, Wenzel K, et al. Functional autoantibodies against G-protein coupled receptors in patients with persistent Long-COVID-19 symptoms. J Transl Autoimmun. 2021;4:100100. doi:10.1016/j.jtauto.2021.100100

237. Lukitsch I, Kehr J, Chaykovska L, et al. Renal Ischemia and Transplantation Predispose to Vascular Constriction Mediated by Angiotensin II Type 1 Receptor-Activating Antibodies. Transplant J. 2012;94(1):8-13. doi:10.1097/TP.0b013e3182529bb7

238. Scherbakov N, Szklarski M, Hartwig J, et al. Peripheral endothelial dysfunction in myalgic encephalomyelitis/chronic fatigue syndrome. ESC Heart Fail. 2020;7(3):1064-1071. doi:10.1002/ehf2.12633

239. Hoffmann C, Zürn A, Bünemann M, Lohse MJ. Conformational changes in G-protein-coupled receptors - the quest for functionally selective conformations is open. Br J Pharmacol. 2008;153(Suppl 1):S358-S366. doi:10.1038/sj.bjp.0707615

240. Newton DJ, Kennedy G, Chan KKF, Lang CC, Belch JJF, Khan F. Large and small artery endothelial dysfunction in chronic fatigue syndrome. Int J Cardiol. 2012;154(3):335-336. doi:10.1016/j.ijcard.2011.10.030

241. Atzori M, Cuevas-Olguin R, Esquivel-Rendon E, et al. Locus Ceruleus Norepinephrine Release: A Central Regulator of CNS Spatio-Temporal Activation? Front Synaptic Neurosci. 2016;8:25. doi:10.3389/fnsyn.2016.00025

242. Loebel M, Strohschein K, Giannini C, et al. Deficient EBV-Specific B- and T-Cell Response in Patients with Chronic Fatigue Syndrome. PLOS ONE. 2014;9(1):e85387. doi:10.1371/journal.pone.0085387 
243. Lee J-S, Lacerda EM, Nacul L, et al. Salivary DNA loads for human herpes viruses 6 and 7 are correlated with disease phenotype in Myalgic Encephalomyelitis/Chronic Fatigue Syndrome. medRxiv. Published online January 11, 2021:2021.01.06.20248486.

doi:10.1101/2021.01.06.20248486

244. Ariza ME. Myalgic Encephalomyelitis/Chronic Fatigue Syndrome: The Human Herpesviruses Are Back! Biomolecules. 2021;11(2):185. doi:10.3390/biom11020185

245. Komaroff AL. Is human herpesvirus- 6 a trigger for chronic fatigue syndrome? J Clin Virol. 2006;37:S39-S46.

246. Zachrisson O, Regland B, Jahreskog M, Jonsson M, Kron M, Gottfries C-G. Treatment with staphylococcus toxoid in fibromyalgia/chronic fatigue syndrome-a randomised controlled trial. Eur J Pain. 2002;6(6):455-466. doi:10.1016/S1090-3801(02)00044-7

247. Zachrisson O, Colque-Navarro P, Gottfries C-G, Regland B, Möllby R. Immune Modulation with a Staphylococcal Preparation in Fibromyalgia/Chronic Fatigue Syndrome: Relation Between Antibody Levels and Clinical Improvement. Eur J Clin Microbiol Infect Dis Off Publ Eur Soc Clin Microbiol. 2004;23:98-105. doi:10.1007/s10096-003-1062-8

248. Mitchell WM. Efficacy of rintatolimod in the treatment of chronic fatigue syndrome/myalgic encephalomyelitis (CFS/ME). Expert Rev Clin Pharmacol. 2016;9(6):755-770. doi:10.1586/17512433.2016.1172960

249. Strayer DR, Young D, Mitchell WM. Effect of disease duration in a randomized Phase III trial of rintatolimod, an immune modulator for Myalgic Encephalomyelitis/Chronic Fatigue Syndrome. PLOS ONE. 2020;15(10):e0240403. doi:10.1371/journal.pone.0240403

250. Tölle M, Freitag H, Antelmann M, et al. Myalgic Encephalomyelitis/Chronic Fatigue Syndrome: Efficacy of Repeat Immunoadsorption. J Clin Med. 2020;9(8):2443. doi:10.3390/jcm9082443

251. Mandarano AH, Maya J, Giloteaux L, et al. Myalgic encephalomyelitis/chronic fatigue syndrome patients exhibit altered T cell metabolism and cytokine associations. J Clin Invest. 2020;130(3):1491-1505. doi:10.1172/JCI132185

252. Milivojevic $M, C$ Che $X$, Bateman $L$, et al. Plasma proteomic profiling suggests an association between antigen driven clonal B cell expansion and ME/CFS. PLOS ONE. 2020;15(7):e0236148. doi:10.1371/journal.pone. 0236148

253. Gow JW, Hagan S, Herzyk P, Cannon C, Behan PO, Chaudhuri A. A gene signature for postinfectious chronic fatigue syndrome. BMC Med Genomics. 2009;2:38. doi:10.1186/1755-87942-38

254. Morrison $\mathrm{L}$, Behan WH, Behan PO. Changes in natural killer cell phenotype in patients with post-viral fatigue syndrome. Clin Exp Immunol. 1991;83(3):441-446. doi:10.1111/j.13652249.1991.tb05658.x

255. Jonsjö MA, Olsson GL, Wicksell RK, Alving K, Holmström L, Andreasson A. The role of lowgrade inflammation in ME/CFS (Myalgic Encephalomyelitis/Chronic Fatigue Syndrome) associations with symptoms. Psychoneuroendocrinology. 2020;113:104578. doi:10.1016/j.psyneuen.2019.104578

256. Buckwalter MS, Wyss-Coray T. Modelling neuroinflammatory phenotypes in vivo. J Neuroinflammation. 2004;1(1):10. doi:10.1186/1742-2094-1-10 
257. Bureta C, Setoguchi T, Saitoh Y, et al. TGF- $\beta$ Promotes the Proliferation of Microglia In Vitro. Brain Sci. 2019;10(1):20. doi:10.3390/brainsci10010020

258. Linnerbauer M, Rothhammer V. Protective Functions of Reactive Astrocytes Following Central Nervous System Insult. Front Immunol. 2020;11. doi:10.3389/fimmu.2020.573256

259. Goss JR, O'Malley ME, Zou L, Styren SD, Kochanek PM, DeKosky ST. Astrocytes Are the Major Source of Nerve Growth Factor Upregulation Following Traumatic Brain Injury in the Rat. Exp Neurol. 1998;149(2):301-309. doi:10.1006/exnr.1997.6712

260. Woodruff MC, Ramonell RP, Lee FE-H, Sanz I. Clinically identifiable autoreactivity is common in severe SARS-CoV-2 Infection. medRxiv. Published online October 28, 2020:2020.10.21.20216192. doi:10.1101/2020.10.21.20216192

261. Blomberg J, Gottfries C-G, Elfaitouri A, Rizwan M, Rosén A. Infection Elicited Autoimmunity and Myalgic Encephalomyelitis/Chronic Fatigue Syndrome: An Explanatory Model. Front Immunol. 2018;9. doi:10.3389/fimmu.2018.00229

262. Goebel A, Krock E, Gentry C, et al. Passive transfer of fibromyalgia symptoms from patients to mice. J Clin Invest. 2021;131(13). doi:10.1172/JCl144201

263. Freitag H, Szklarski M, Lorenz S, et al. Autoantibodies to Vasoregulative G-Protein-Coupled Receptors Correlate with Symptom Severity, Autonomic Dysfunction and Disability in Myalgic Encephalomyelitis/Chronic Fatigue Syndrome. J Clin Med. 2021;10(16):3675. doi:10.3390/jcm10163675

264. Bynke A, Julin P, Gottfries C-G, Heidecke H, Scheibenbogen C, Bergquist J. Autoantibodies to beta-adrenergic and muscarinic cholinergic receptors in Myalgic Encephalomyelitis (ME) patients - A validation study in plasma and cerebrospinal fluid from two Swedish cohorts. Brain Behav Immun - Health. 2020;7:100107. doi:10.1016/j.bbih.2020.100107

265. Fujii H, Sato W, Kimura Y, et al. Altered Structural Brain Networks Related to Adrenergic/Muscarinic Receptor Autoantibodies in Chronic Fatigue Syndrome. J Neuroimaging. 2020;30(6):822-827. doi:10.1111/jon.12751

266. Hohberger B, Harrer T, Mardin C, et al. Neutralization of Autoantibodies Targeting G-Protein Coupled Receptors Improves Capillary Impairment and Fatigue Symptoms after COVID-19 Infection. Social Science Research Network; 2021. Accessed August 31, 2021. https://papers.ssrn.com/abstract $=3879488$

267. Gadea A, Schinelli S, Gallo V. Endothelin-1 Regulates Astrocyte Proliferation and Reactive Gliosis via a JNK/c-Jun Signaling Pathway. J Neurosci. 2008;28(10):2394-2408. doi:10.1523/JNEUROSCI.5652-07.2008

268. Koyama Y. Endothelin ETB Receptor-Mediated Astrocytic Activation: Pathological Roles in Brain Disorders. Int J Mol Sci. 2021;22(9):4333. doi:10.3390/ijms22094333

269. Sharma D, Farrar JD. Adrenergic regulation of immune cell function and inflammation. Semin Immunopathol. 2020;42(6):709-717. doi:10.1007/s00281-020-00829-6

270. Wang $D$. The essential role of $G$ protein-coupled receptor (GPCR) signaling in regulating $T$ cell immunity. Immunopharmacol Immunotoxicol. 2018;40(3):187-192.

doi:10.1080/08923973.2018.1434792 
271. Grayston R, Czanner G, Elhadd K, et al. A systematic review and meta-analysis of the prevalence of small fiber pathology in fibromyalgia: Implications for a new paradigm in fibromyalgia etiopathogenesis. Semin Arthritis Rheum. 2019;48(5):933-940.

doi:10.1016/j.semarthrit.2018.08.003

272. Oaklander AL, Nolano M. Scientific Advances in and Clinical Approaches to Small-Fiber Polyneuropathy: A Review. JAMA Neurol. 2019;76(10):1240-1251.

doi:10.1001/jamaneurol.2019.2917

273. Joseph P, Arevalo C, Oliveira RKF, et al. Insights From Invasive Cardiopulmonary Exercise Testing of Patients With Myalgic Encephalomyelitis/Chronic Fatigue Syndrome. Chest. Published online February 10, 2021. doi:10.1016/j.chest.2021.01.082

274. Giannoccaro MP, Cossins J, Sørland K, Fluge $\varnothing$, Vincent A. Searching for Serum Antibodies to Neuronal Proteins in Patients With Myalgic Encephalopathy/Chronic Fatigue Syndrome. Clin Ther. 2019;41(5):836-847. doi:10.1016/j.clinthera.2019.04.001

275. Ikeshima-Kataoka H. Neuroimmunological Implications of AQP4 in Astrocytes. Int J Mol Sci. 2016;17(8). doi:10.3390/ijms17081306

276. Baecher-Allan C, Kaskow BJ, Weiner HL. Multiple Sclerosis: Mechanisms and Immunotherapy. Neuron. 2018;97(4):742-768. doi:10.1016/j.neuron.2018.01.021

277. Dong Y, Yong VW. When encephalitogenic T cells collaborate with microglia in multiple sclerosis. Nat Rev Neurol. 2019;15(12):704-717. doi:10.1038/s41582-019-0253-6

278. Nguyen T, Johnston S, Chacko A, et al. Novel characterisation of mast cell phenotypes from peripheral blood mononuclear cells in chronic fatigue syndrome/myalgic encephalomyelitis patients. Asian Pac J Allergy Immunol. 2017;35(2):75-81. doi:10.12932/ap0771

279. Frieri M. Mast Cell Activation Syndrome. Clin Rev Allergy Immunol. 2018;54(3):353-365. doi:10.1007/s12016-015-8487-6

280. Rozniecki JJ, Dimitriadou V, Lambracht-Hall M, Pang X, Theoharides TC. Morphological and functional demonstration of rat dura mater mast cell-neuron interactions in vitro and in vivo. Brain Res. 1999;849(1-2):1-15. doi:10.1016/s0006-8993(99)01855-7

281. Skaper SD, Facci L, Zusso M, Giusti P. Neuroinflammation, Mast Cells, and Glia: Dangerous Liaisons. Neurosci Rev J Bringing Neurobiol Neurol Psychiatry. 2017;23(5):478-498. doi:10.1177/1073858416687249

282. Zhang $\mathrm{X}$, Wang $\mathrm{Y}$, Dong $\mathrm{H}, \mathrm{Xu} \mathrm{Y}$, Zhang S. Induction of Microglial Activation by Mediators Released from Mast Cells. Cell Physiol Biochem Int J Exp Cell Physiol Biochem Pharmacol. 2016;38(4):1520-1531. doi:10.1159/000443093

283. Zhang X, Yao H, Qian Q, Li N, Jin W, Qian Y. Cerebral Mast Cells Participate In Postoperative Cognitive Dysfunction by Promoting Astrocyte Activation. Cell Physiol Biochem Int J Exp Cell Physiol Biochem Pharmacol. 2016;40(1-2):104-116. doi:10.1159/000452528

284. Sarno EL. Mast cells as mediators of astrocyte morphology associated with neuroinflammatory disease states. J Immunol. 2020;204(1 Supplement):64.29-64.29. 
285. Theoharides TC. The impact of psychological stress on mast cells. Ann Allergy Asthma Immunol Off Publ Am Coll Allergy Asthma Immunol. 2020;125(4):388-392.

doi:10.1016/j.anai.2020.07.007

286. Theoharides TC, Konstantinidou AD. Corticotropin-releasing hormone and the blood-brainbarrier. Front Biosci J Virtual Libr. 2007;12:1615-1628. doi:10.2741/2174

287. Esposito P, Gheorghe D, Kandere K, et al. Acute stress increases permeability of the bloodbrain-barrier through activation of brain mast cells. Brain Res. 2001;888(1):117-127. doi:10.1016/s0006-8993(00)03026-2

288. Kempuraj D, Mentor S, Thangavel R, et al. Mast Cells in Stress, Pain, Blood-Brain Barrier, Neuroinflammation and Alzheimer's Disease. Front Cell Neurosci. 2019;13:54. doi:10.3389/fncel.2019.00054

289. Hu KK, Bruce MA, Butte MJ. Spatiotemporally and mechanically controlled triggering of mast cells using atomic force microscopy. Immunol Res. 2014;58(2):211-217. doi:10.1007/s12026014-8510-7

290. Theoharides TC, Papaliodis D, Tagen M, Konstantinidou A, Kempuraj D, Clemons A. Chronic fatigue syndrome, mast cells, and tricyclic antidepressants. J Clin Psychopharmacol. 2005;25(6):515-520. doi:10.1097/01.jcp.0000193483.89260.a7

291. Shibao C, Arzubiaga C, Roberts $L$, et al. Hyperadrenergic postural tachycardia syndrome in mast cell activation disorders. Hypertens Dallas Tex 1979. 2005;45(3):385-390. doi:10.1161/01.HYP.0000158259.68614.40

292. Lyons JJ, Sun G, Stone KD, et al. Mendelian inheritance of elevated serum tryptase associated with atopy and connective tissue abnormalities. J Allergy Clin Immunol. 2014;133(5):14711474. doi:10.1016/j.jaci.2013.11.039

293. Lyons JJ, Yu X, Hughes JD, et al. Elevated basal serum tryptase identifies a multisystem disorder associated with increased TPSAB1 copy number. Nat Genet. 2016;48(12):1564-1569. doi:10.1038/ng.3696

294. Weinstock LB, Brook JB, Myers TL, Goodman B. Successful treatment of postural orthostatic tachycardia and mast cell activation syndromes using naltrexone, immunoglobulin and antibiotic treatment. BMJ Case Rep. 2018;2018:bcr2017221405. doi:10.1136/bcr-2017221405

295. Glynne P, Tahmasebi N, Gant V, Gupta R. Long-COVID following mild SARS CoV-2 infection: characteristic $T$ cell alterations and response to antihistamines. medRxiv. Published online June 7, 2021:2021.06.06.21258272. doi:10.1101/2021.06.06.21258272

296. Kashi AA, Davis RW, Phair RD. The IDO Metabolic Trap Hypothesis for the Etiology of ME/CFS. Diagnostics. 2019;9(3):82. doi:10.3390/diagnostics9030082

297. Eroğlu I, Eroğlu BÇ, Güven GS. Altered tryptophan absorption and metabolism could underlie long-term symptoms in survivors of coronavirus disease 2019 (COVID-19). Nutr Burbank Los Angel Cty Calif. 2021;90:111308. doi:10.1016/j.nut.2021.111308

298. Davis I, Liu A. What is the tryptophan kynurenine pathway and why is it important to neurotherapy? Expert Rev Neurother. 2015;15(7):719-721.

doi:10.1586/14737175.2015.1049999 
299. Kindler J, Lim CK, Weickert CS, et al. Dysregulation of kynurenine metabolism is related to proinflammatory cytokines, attention, and prefrontal cortex volume in schizophrenia. $\mathrm{Mol}$ Psychiatry. 2020;25(11):2860-2872. doi:10.1038/s41380-019-0401-9

300. Chiappelli J, Pocivavsek A, Nugent KL, et al. Stress-Induced Increase in Kynurenic Acid as a Potential Biomarker for Patients With Schizophrenia and Distress Intolerance. JAMA Psychiatry. 2014;71(7):761-768. doi:10.1001/jamapsychiatry.2014.243

301. Pocivavsek A, Notarangelo FM, Wu HQ, Bruno JP, Schwarcz R. Handbook of Behavioral Neuroscience. Published online 2016.

302. Myint A-M, Schwarz MJ, Müller N. The role of the kynurenine metabolism in major depression. J Neural Transm Vienna Austria 1996. 2012;119(2):245-251. doi:10.1007/s00702011-0741-3

303. O'Farrell K, Harkin A. Stress-related regulation of the kynurenine pathway: Relevance to neuropsychiatric and degenerative disorders. Neuropharmacology. 2017;112(Pt B):307-323. doi:10.1016/j.neuropharm.2015.12.004

304. Won E, Kim Y-K. Stress, the Autonomic Nervous System, and the Immune-kynurenine Pathway in the Etiology of Depression. Curr Neuropharmacol. 2016;14(7):665-673. doi:10.2174/1570159X14666151208113006

305. Yamashita M. Potential Role of Neuroactive Tryptophan Metabolites in Central Fatigue: Establishment of the Fatigue Circuit. Int J Tryptophan Res IJTR. 2020;13. doi:10.1177/1178646920936279

306. Németh H, Toldi J, Vécsei L. Role of kynurenines in the central and peripheral nervous systems. Curr Neurovasc Res. 2005;2(3):249-260. doi:10.2174/1567202054368326

307. Yamamoto T, Azechi H, Board M. Essential role of excessive tryptophan and its neurometabolites in fatigue. Can J Neurol Sci J Can Sci Neurol. 2012;39(1):40-47. doi:10.1017/s031716710001266x

308. Anderson $\mathrm{G}$, Maes $\mathrm{M}$, Berk M. Biological underpinnings of the commonalities in depression, somatization, and Chronic Fatigue Syndrome. Med Hypotheses. 2012;78(6):752-756. doi:10.1016/j.mehy.2012.02.023

309. Maes M, Twisk FNM, Ringel K. Inflammatory and cell-mediated immune biomarkers in myalgic encephalomyelitis/chronic fatigue syndrome and depression: inflammatory markers are higher in myalgic encephalomyelitis/chronic fatigue syndrome than in depression. Psychother Psychosom. 2012;81(5):286-295. doi:10.1159/000336803

310. Capuron L, Miller AH. Immune System to Brain Signaling: Neuropsychopharmacological Implications. Pharmacol Ther. 2011;130(2):226-238. doi:10.1016/j.pharmthera.2011.01.014

311. Dostal CR, Carson Sulzer M, Kelley KW, Freund GG, McCusker RH. Glial and tissue-specific regulation of Kynurenine Pathway dioxygenases by acute stress of mice. Neurobiol Stress. 2017;7:1-15. doi:10.1016/j.ynstr.2017.02.002

312. Thomas T, Stefanoni D, Reisz JA, et al. COVID-19 infection alters kynurenine and fatty acid metabolism, correlating with IL-6 levels and renal status. JCI Insight. 2020;5(14). doi:10.1172/jci.insight.140327 
313. Holmes E, Wist J, Masuda R, et al. Incomplete Systemic Recovery and Metabolic Phenoreversion in Post-Acute-Phase Nonhospitalized COVID-19 Patients: Implications for Assessment of Post-Acute COVID-19 Syndrome. J Proteome Res. 2021;20(6):3315-3329. doi:10.1021/acs.jproteome.1c00224

314. Wirth KJ, Scheibenbogen C. Pathophysiology of skeletal muscle disturbances in Myalgic Encephalomyelitis/Chronic Fatigue Syndrome (ME/CFS). J Transl Med. 2021;19(1):162. doi:10.1186/s12967-021-02833-2

315. Lim Y, Cho I-T, Rennke HG, Cho G. Beta-adrenergic receptor signaling regulates ERmitochondria contacts. bioRxiv. Published online March 25, 2021:2020.05.11.088815. doi:10.1101/2020.05.11.088815

316. JCl Insight - A map of metabolic phenotypes in patients with myalgic encephalomyelitis/chronic fatigue syndrome. Accessed September 19, 2021. https://insight.jci.org/articles/view/149217

317. Buoncervello M, Maccari S, Ascione B, et al. Inflammatory cytokines associated with cancer growth induce mitochondria and cytoskeleton alterations in cardiomyocytes. J Cell Physiol. 2019;234(11):20453-20468. doi:10.1002/jcp.28647

318. Paul BD, Lemle MD, Komaroff AL, Snyder SH. Redox imbalance links COVID-19 and myalgic encephalomyelitis/chronic fatigue syndrome. Proc Natl Acad Sci. 2021;118(34). doi:10.1073/pnas.2024358118

319. van Horssen J, van Schaik P, Witte M. Inflammation and mitochondrial dysfunction: A vicious circle in neurodegenerative disorders? Neurosci Lett. 2019;710:132931. doi:10.1016/j.neulet.2017.06.050

320. Missiroli S, Genovese I, Perrone M, Vezzani B, Vitto VAM, Giorgi C. The Role of Mitochondria in Inflammation: From Cancer to Neurodegenerative Disorders. J Clin Med. 2020;9(3):740. doi:10.3390/jcm9030740

321. Stefano GB, Büttiker P, Weissenberger S, Martin A, Ptacek R, Kream RM. Editorial: The Pathogenesis of Long-Term Neuropsychiatric COVID-19 and the Role of Microglia, Mitochondria, and Persistent Neuroinflammation: A Hypothesis. Med Sci Monit Int Med J Exp Clin Res. 2021;27:e933015. doi:10.12659/MSM.933015

322. Culmsee C, Michels S, Scheu S, Arolt V, Dannlowski U, Alferink J. Mitochondria, Microglia, and the Immune System-How Are They Linked in Affective Disorders? Front Psychiatry. 2018;9:739. doi:10.3389/fpsyt.2018.00739

323. Morris G, Maes M. Mitochondrial dysfunctions in myalgic encephalomyelitis/chronic fatigue syndrome explained by activated immuno-inflammatory, oxidative and nitrosative stress pathways. Metab Brain Dis. 2014;29(1):19-36. doi:10.1007/s11011-013-9435-x

324. Toogood PL, Clauw DJ, Phadke S, Hoffman D. Myalgic encephalomyelitis/chronic fatigue syndrome (ME/CFS): Where will the drugs come from? Pharmacol Res. 2021;165:105465. doi:10.1016/j.phrs.2021.105465

325. Costa DC, Tannock C, Brostoff J. Brainstem perfusion is impaired in chronic fatigue syndrome. QJM Mon J Assoc Physicians. 1995;88(11):767-773. 
326. Yoshiuchi K, Farkas J, Natelson BH. Patients with chronic fatigue syndrome have reduced absolute cortical blood flow. Clin Physiol Funct Imaging. 2006;26(2):83-86. doi:10.1111/j.1475097X.2006.00649.x

327. Ichise M, Salit IE, Abbey SE, et al. Assessment of regional cerebral perfusion by $99 \mathrm{Tcm}-\mathrm{HMPAO}$ SPECT in chronic fatigue syndrome. Nucl Med Commun. 1992;13(10):767-772.

328. Tirelli U, Chierichetti F, Tavio M, et al. Brain positron emission tomography (PET) in chronic fatigue syndrome: preliminary data. Am J Med. 1998;105(3A):54S-58S. doi:10.1016/s00029343(98)00179-x

329. Fischler B, D'Haenen H, Cluydts R, et al. Comparison of 99mTc HMPAO SPECT Scan between Chronic Fatigue Syndrome, Major Depression and Healthy Controls: An Exploratory Study of Clinical Correlates of Regional Cerebral Blood Flow. Neuropsychobiology. 1996;34(4):175-183. doi:10.1159/000119307

330. Siessmeier T, Nix WA, Hardt J, Schreckenberger M, Egle UT, Bartenstein P. Observer independent analysis of cerebral glucose metabolism in patients with chronic fatigue syndrome. J Neurol Neurosurg Psychiatry. 2003;74(7):922-928. doi:10.1136/jnnp.74.7.922

331. Biswal B, Kunwar P, Natelson BH. Cerebral blood flow is reduced in chronic fatigue syndrome as assessed by arterial spin labeling. J Neurol Sci. 2011;301(1-2):9-11. doi:10.1016/j.jns.2010.11.018

332. van Campen C (Linda) MC, Verheugt FWA, Rowe PC, Visser FC. Cerebral blood flow is reduced in ME/CFS during head-up tilt testing even in the absence of hypotension or tachycardia: $\mathrm{A}$ quantitative, controlled study using Doppler echography. Clin Neurophysiol Pract. 2020;5:5058. doi:10.1016/j.cnp.2020.01.003

333. Stewart JM, Medow MS, Messer ZR, Baugham IL, Terilli C, Ocon AJ. Postural neurocognitive and neuronal activated cerebral blood flow deficits in young chronic fatigue syndrome patients with postural tachycardia syndrome. Am J Physiol - Heart Circ Physiol. 2012;302(5):H1185-H1194. doi:10.1152/ajpheart.00994.2011

334. Medow MS, Sood S, Messer Z, Dzogbeta S, Terilli C, Stewart JM. Phenylephrine alteration of cerebral blood flow during orthostasis: effect on $\mathrm{n}$-back performance in chronic fatigue syndrome. J Appl Physiol. 2014;117(10):1157-1164. doi:10.1152/japplphysiol.00527.2014

335. Campen LMC van, Rowe PC, Visser FC. The Myalgic Encephalomyelitis/Chronic Fatigue Syndrome Patients with Joint Hypermobility Show Larger Cerebral Blood Flow Reductions during Orthostatic Stress Testing Than Patients without Hypermobility: A Case Control Study. Med Res Arch. 2021;9(6). doi:10.18103/mra.v9i6.2494

336. Najjar S, Najjar A, Chong DJ, et al. Central nervous system complications associated with SARSCoV-2 infection: integrative concepts of pathophysiology and case reports. $J$ Neuroinflammation. 2020;17. doi:10.1186/s12974-020-01896-0

337. Cipolla MJ. Control of Cerebral Blood Flow. Morgan \& Claypool Life Sciences; 2009. Accessed June 16, 2021. https://www.ncbi.nlm.nih.gov/books/NBK53082/

338. Araque A. Astrocyte-neuron signaling in the brain--implications for disease. Curr Opin Investig Drugs Lond Engl 2000. 2006;7(7):619-624. 
339. Frith J, Zalewski P, Klawe JJ, et al. Impaired blood pressure variability in chronic fatigue syndrome--a potential biomarker. QJM Mon J Assoc Physicians. 2012;105(9):831-838. doi:10.1093/qjmed/hcs085

340. Fukuma N, Kato K, Munakata K, et al. Baroreflex mechanisms and response to exercise in patients with heart disease. Clin Physiol Funct Imaging. 2012;32(4):305-309. doi:10.1111/j.1475-097X.2012.01127.x

341. Becker BK, Tian C, Zucker IH, Wang H-J. Influence of brain-derived neurotrophic factortyrosine receptor kinase $B$ signalling in the nucleus tractus solitarius on baroreflex sensitivity in rats with chronic heart failure. J Physiol. 2016;594(19):5711-5725. doi:10.1113/JP272318

342. Bragée B, Michos A, Drum B, Fahlgren M, Szulkin R, Bertilson BC. Signs of Intracranial Hypertension, Hypermobility, and Craniocervical Obstructions in Patients With Myalgic Encephalomyelitis/Chronic Fatigue Syndrome. Front Neurol. 2020;11. doi:10.3389/fneur.2020.00828

343. Higgins N, Pickard J, Lever A. Lumbar puncture, chronic fatigue syndrome and idiopathic intracranial hypertension: a cross-sectional study. JRSM Short Rep. 2013;4(12):2042533313507920. doi:10.1177/2042533313507920

344. Proal AD, VanElzakker MB. Long COVID or Post-acute Sequelae of COVID-19 (PASC): An Overview of Biological Factors That May Contribute to Persistent Symptoms. Front Microbiol. 2021;12. doi:10.3389/fmicb.2021.698169

345. Henderson FC, Austin C, Benzel E, et al. Neurological and spinal manifestations of the EhlersDanlos syndromes. Am J Med Genet C Semin Med Genet. 2017;175(1):195-211. doi:10.1002/ajmg.c.31549

346. bobbyjonescsf. Venous Stenting in Intracranial Hypertension - Dr. Kenneth Liu.; 2016. Accessed June 21, 2021. https://vimeo.com/190164038

347. Davalos D, Ryu JK, Merlini M, et al. Fibrinogen-induced perivascular microglial clustering is required for the development of axonal damage in neuroinflammation. Nat Commun. 2012;3(1):1-15.

348. Brinker T, Stopa E, Morrison J, Klinge P. A new look at cerebrospinal fluid circulation. Fluids Barriers CNS. 2014;11(1):10. doi:10.1186/2045-8118-11-10

349. Buchwald D, Cheney PR, Peterson DL, et al. A chronic illness characterized by fatigue, neurologic and immunologic disorders, and active human herpesvirus type 6 infection. Ann Intern Med. 1992;116(2):103-113. doi:10.7326/0003-4819-116-2-103

350. Małkiewicz MA, Szarmach A, Sabisz A, Cubała WJ, Szurowska E, Winklewski PJ. Blood-brain barrier permeability and physical exercise. J Neuroinflammation. 2019;16:15. doi:10.1186/s12974-019-1403-x

351. Lubell J. Could High-Dose Thiamine (B-1) Help with ME/CFS, Fibromyalgia, and the Neurological Complications of Ehlers-Danlos Syndrome? Health Rising. Published April 15, 2021. Accessed June 21, 2021. https://www.healthrising.org/blog/2021/04/15/thiamine-b-1chronic-fatigue-syndrome-fibromyalgia/

352. Lubell J. Nearly $2 / 3$ rds of Survey Respondents Report Large Benefits from High-Dose Thiamine (B-1). Health Rising. Published June 3, 2021. Accessed June 21, 2021. 
https://www.healthrising.org/blog/2021/06/02/fibromyalgia-chronic-fatigue-syndromebenefit-high-dose-thiamine/

353. Agulhon C, Boyt KM, Xie AX, Friocourt F, Roth BL, McCarthy KD. Modulation of the autonomic nervous system and behaviour by acute glial cell Gq protein-coupled receptor activation in vivo. J Physiol. 2013;591(22):5599-5609. doi:10.1113/jphysiol.2013.261289

354. Barnden LR, Kwiatek R, Crouch B, Burnet R, Del Fante P. Autonomic correlations with MRI are abnormal in the brainstem vasomotor centre in Chronic Fatigue Syndrome. Neurolmage Clin. 2016;11:530-537. doi:10.1016/j.nicl.2016.03.017

355. Kraynak TE, Marsland AL, Wager TD, Gianaros PJ. Functional neuroanatomy of peripheral inflammatory physiology: A meta-analysis of human neuroimaging studies. Neurosci Biobehav Rev. 2018;94:76-92. doi:10.1016/j.neubiorev.2018.07.013

356. Wyller VB, Barbieri R, Thaulow E, Saul JP. Enhanced vagal withdrawal during mild orthostatic stress in adolescents with chronic fatigue. Ann Noninvasive Electrocardiol Off J Int Soc Holter Noninvasive Electrocardiol Inc. 2008;13(1):67-73. doi:10.1111/j.1542-474X.2007.00202.x

357. Martínez-Martínez L-A, Mora T, Vargas A, Fuentes-Iniestra M, Martínez-Lavín M. Sympathetic nervous system dysfunction in fibromyalgia, chronic fatigue syndrome, irritable bowel syndrome, and interstitial cystitis: a review of case-control studies. J Clin Rheumatol Pract Rep Rheum Musculoskelet Dis. 2014;20(3):146-150. doi:10.1097/RHU.0000000000000089

358. JCM | Free Full-Text | Reduced Parasympathetic Reactivation during Recovery from Exercise in Myalgic Encephalomyelitis/Chronic Fatigue Syndrome | HTML. Accessed October 26, 2021. https://www.mdpi.com/2077-0383/10/19/4527/htm

359. Tracey KJ. The inflammatory reflex. Nature. 2002;420(6917):853-859. doi:10.1038/nature01321

360. Vernino S, Stiles LE. Autoimmunity in postural orthostatic tachycardia syndrome: Current understanding. Auton Neurosci Basic Clin. 2018;215:78-82. doi:10.1016/j.autneu.2018.04.005

361. Durosier LD, Herry $\mathrm{CL}$, Cortes $\mathrm{M}$, et al. Does heart rate variability reflect the systemic inflammatory response in a fetal sheep model of lipopolysaccharide-induced sepsis? Physiol Meas. 2015;36(10):2089-2102. doi:10.1088/0967-3334/36/10/2089

362. Herry CL, Cortes M, Wu H-T, et al. Temporal Patterns in Sheep Fetal Heart Rate Variability Correlate to Systemic Cytokine Inflammatory Response: A Methodological Exploration of Monitoring Potential Using Complex Signals Bioinformatics. PloS One. 2016;11(4):e0153515. doi:10.1371/journal.pone.0153515

363. Rodriguez LST, Pou C, Lakshmikanth T, et al. Achieving symptom relief in patients with Myalgic encephalomyelitis by targeting the neuro-immune interface and inducing disease tolerance. bioRxiv. Published online February 28, 2020:2020.02.20.958249. doi:10.1101/2020.02.20.958249

364. Naviaux RK, Naviaux JC, Li K, et al. Metabolic features of chronic fatigue syndrome. Proc Natl Acad Sci. 2016;113(37):E5472-E5480.

365. Magistretti PJ, Allaman I. Lactate in the brain: from metabolic end-product to signalling molecule. Nat Rev Neurosci. 2018;19(4):235-249. doi:10.1038/nrn.2018.19 
366. Riske L, Thomas RK, Baker GB, Dursun SM. Lactate in the brain: an update on its relevance to brain energy, neurons, glia and panic disorder. Ther Adv Psychopharmacol. 2017;7(2):85-89. doi:10.1177/2045125316675579

367. Nijs J, Meeus M, Van Oosterwijck J, et al. In the mind or in the brain? Scientific evidence for central sensitisation in chronic fatigue syndrome. Eur J Clin Invest. 2012;42(2):203-212. doi:10.1111/j.1365-2362.2011.02575.x

368. Arborelius L, Owens MJ, Plotsky PM, Nemeroff CB. The role of corticotropin-releasing factor in depression and anxiety disorders. J Endocrinol. 1999;160(1):1-12. doi:10.1677/joe.0.1600001

369. Jessop DS, Harbuz MS, Lightman SL. CRH in chronic inflammatory stress. Peptides. 2001;22(5):803-807. doi:10.1016/s0196-9781(01)00394-1

370. Wang W, Ji P, Dow KE. Corticotropin-releasing hormone induces proliferation and TNF-alpha release in cultured rat microglia via MAP kinase signalling pathways. J Neurochem. 2003;84(1):189-195. doi:10.1046/j.1471-4159.2003.01544.x

371. Stevens SL, Shaw TE, Dykhuizen E, et al. Reduced cerebral injury in CRH-R1 deficient mice after focal ischemia: a potential link to microglia and atrocytes that express CRH-R1. J Cereb Blood Flow Metab Off J Int Soc Cereb Blood Flow Metab. 2003;23(10):1151-1159. doi:10.1097/01.WCB.0000086957.72078.D4

372. Jiang Y, Peng T, Gaur U, et al. Role of Corticotropin Releasing Factor in the Neuroimmune Mechanisms of Depression: Examination of Current Pharmaceutical and Herbal Therapies. Front Cell Neurosci. 2019;13. doi:10.3389/fncel.2019.00290

373. Karalis KP, Venihaki M, Zhao J, Vlerken LE van, Chandras C. NF-KB Participates in the Corticotropin-releasing, Hormone-induced Regulation of the Pituitary Proopiomelanocortin Gene *. J Biol Chem. 2004;279(12):10837-10840. doi:10.1074/jbc.M313063200

374. Lezoualc'h F, Engert S, Berning B, Behl C. Corticotropin-releasing hormone-mediated neuroprotection against oxidative stress is associated with the increased release of nonamyloidogenic amyloid beta precursor protein and with the suppression of nuclear factorkappaB. Mol Endocrinol Baltim Md. 2000;14(1):147-159. doi:10.1210/mend.14.1.0403

375. Neufeld-Cohen A, Kelly PAT, Paul ED, et al. Chronic activation of corticotropin-releasing factor type 2 receptors reveals a key role for $5-\mathrm{HT} 1 \mathrm{~A}$ receptor responsiveness in mediating behavioral and serotonergic responses to stressful challenge. Biol Psychiatry. 2012;72(6):437447. doi:10.1016/j.biopsych.2012.05.005

376. Pereira G, Gillies H, Chanda S, et al. Acute Corticotropin-Releasing Factor Receptor Type 2 Agonism Results in Sustained Symptom Improvement in Myalgic Encephalomyelitis/Chronic Fatigue Syndrome. Front Syst Neurosci. 2021;15:84. doi:10.3389/fnsys.2021.698240

377. Matchett BJ, Grinberg LT, Theofilas P, Murray ME. The mechanistic link between selective vulnerability of the locus coeruleus and neurodegeneration in Alzheimer's disease. Acta Neuropathol (Berl). 2021;141(5):631-650. doi:10.1007/s00401-020-02248-1

378. Borodovitsyna O, Joshi N, Chandler D. Persistent Stress-Induced Neuroplastic Changes in the Locus Coeruleus/Norepinephrine System. Neural Plast. 2018;2018:e1892570. doi:10.1155/2018/1892570 
379. Ross JA, Van Bockstaele EJ. The Locus Coeruleus- Norepinephrine System in Stress and Arousal: Unraveling Historical, Current, and Future Perspectives. Front Psychiatry. 2021;11. doi:10.3389/fpsyt.2020.601519

380. Morrison JH, Grzanna R, Molliver ME, Coyle JT. The distribution and orientation of noradrenergic fibers in neocortex of the rat: an immunofluorescence study. J Comp Neurol. 1978;181(1):17-39. doi:10.1002/cne.901810103

381. Sara SJ. The locus coeruleus and noradrenergic modulation of cognition. Nat Rev Neurosci. 2009;10(3):211-223. doi:10.1038/nrn2573

382. Swanson LW, Hartman BK. The central adrenergic system. An immunofluorescence study of the location of cell bodies and their efferent connections in the rat utilizing dopamine-betahydroxylase as a marker. J Comp Neurol. 1975;163(4):467-505. doi:10.1002/cne.901630406

383. Liu Y, Zhao J, Fan X, Guo W. Dysfunction in Serotonergic and Noradrenergic Systems and Somatic Symptoms in Psychiatric Disorders. Front Psychiatry. 2019;10. doi:10.3389/fpsyt.2019.00286

384. Reyes BAS, Valentino RJ, Xu G, Van Bockstaele EJ. Hypothalamic projections to locus coeruleus neurons in rat brain. Eur J Neurosci. 2005;22(1):93-106. doi:10.1111/j.14609568.2005.04197.x

385. Flak JN, Myers B, Solomon MB, McKlveen JM, Krause EG, Herman JP. Role of Paraventricular Nucleus-projecting Norepinephrine/Epinephrine Neurons in Acute and Chronic Stress. Eur J Neurosci. 2014;39(11):1903-1911. doi:10.1111/ejn.12587

386. Maksoud R, du Preez S, Eaton-Fitch N, et al. A systematic review of neurological impairments in myalgic encephalomyelitis/ chronic fatigue syndrome using neuroimaging techniques. PLoS ONE. 2020;15(4). doi:10.1371/journal.pone.0232475

387. Silva LS, Joao RB, Nogueira MH, et al. Functional and microstructural brain abnormalities, fatigue, and cognitive dysfunction after mild COVID-19. medRxiv. Published online March 24, 2021:2021.03.20.21253414. doi:10.1101/2021.03.20.21253414

388. Hugon J, Msika E-F, Queneau M, Farid K, Paquet C. Long COVID: cognitive complaints (brain fog) and dysfunction of the cingulate cortex. J Neurol. Published online June 18, 2021. doi:10.1007/s00415-021-10655-x

389. Naegeli C, Zeffiro T, Piccirelli M, et al. Locus Coeruleus Activity Mediates Hyperresponsiveness in Posttraumatic Stress Disorder. Biol Psychiatry. 2018;83(3):254-262.

doi:10.1016/j.biopsych.2017.08.021

390. Morris G, Maes M. Increased nuclear factor-kB and loss of $\mathrm{p} 53$ are key mechanisms in Myalgic Encephalomyelitis/chronic fatigue syndrome (ME/CFS). Med Hypotheses. 2012;79(5):607-613. doi:10.1016/j.mehy.2012.07.034

391. Morris G, Maes M, Berk M, Puri BK. Myalgic encephalomyelitis or chronic fatigue syndrome: how could the illness develop? Metab Brain Dis. 2019;34(2):385-415. doi:10.1007/s11011019-0388-6

392. Yang X, Li F, Liu Y, Li D, Li J. Study on the Correlation Between NF-kB and Central Fatigue. J Mol Neurosci. Published online February 14, 2021. doi:10.1007/s12031-021-01803-z 
393. Fagermoen E, Sulheim D, Winger A, et al. Effects of low-dose clonidine on cardiovascular and autonomic variables in adolescents with chronic fatigue: a randomized controlled trial. $B M C$ Pediatr. 2015;15:117. doi:10.1186/s12887-015-0428-2

394. Rowe PC, Barron DF, Calkins H, Maumenee IH, Tong PY, Geraghty MT. Orthostatic intolerance and chronic fatigue syndrome associated with Ehlers-Danlos syndrome. J Pediatr. 1999;135(4):494-499. doi:10.1016/S0022-3476(99)70173-3

395. Barron DF, Cohen BA, Geraghty MT, Violand R, Rowe PC. Joint hypermobility is more common in children with chronic fatigue syndrome than in healthy controls. J Pediatr. 2002;141(3):421425. doi:10.1067/mpd.2002.127496

396. OSF Preprints | Bragee Bertilson et al. - ME CFS and Intracranial Hypertension now published please redirect to Frontiers. Accessed June 16, 2021. https://osf.io/qwn5h/

397. Castori M, Celletti C, Camerota F, Grammatico P. Chronic fatigue syndrome is commonly diagnosed in patients with Ehlers-Danlos syndrome hypermobility type/joint hypermobility syndrome. Clin Exp Rheumatol. 2011;29(3):597-598.

398. Could Craniocervical Instability Be Causing ME/CFS, Fibromyalgia \& POTS? Pt I - The Spinal Series - Health Rising. Accessed June 16, 2021.

https://webcache.googleusercontent.com/search?q=cache:Nrl71u7d0GAJ:https://www.healt hrising.org/blog/2019/02/27/brainstem-compression-chronic-fatigue-syndrome-me-cfsfibromyalgia-pots-craniocervical-instability $/+\& c d=1 \& h l=d e \& c t=c \mid n k \& g l=d e \& c l i e n t=f i r e f o x-b-d$

399. Voermans NC, Knoop H, van de Kamp N, Hamel BC, Bleijenberg G, van Engelen BG. Fatigue is a frequent and clinically relevant problem in Ehlers-Danlos Syndrome. Semin Arthritis Rheum. 2010;40(3):267-274. doi:10.1016/j.semarthrit.2009.08.003

400. Eccles JA, Thompson B, Themelis K, et al. Beyond bones: The relevance of variants of connective tissue (hypermobility) to fibromyalgia, ME/CFS and controversies surrounding diagnostic classification: an observational study. Clin Med. 2021;21(1):53-58. doi:10.7861/clinmed.2020-0743

401. Riley B. The Many Facets of Hypermobile Ehlers-Danlos Syndrome. J Osteopath Med. 2020;120(1):30-32. doi:10.7556/jaoa.2020.012

402. Chiarelli N, Ritelli M, Zoppi N, Colombi M. Cellular and Molecular Mechanisms in the Pathogenesis of Classical, Vascular, and Hypermobile Ehlers-Danlos Syndromes. Genes. 2019;10(8). doi:10.3390/genes10080609

403. Sellner J, Simon F, Meyding-Lamade U, Leib SL. Herpes-simplex virus encephalitis is characterized by an early MMP-9 increase and collagen type IV degradation. Brain Res. 2006;1125(1):155-162. doi:10.1016/j.brainres.2006.09.093

404. Singh B, Fleury C, Jalalvand F, Riesbeck K. Human pathogens utilize host extracellular matrix proteins laminin and collagen for adhesion and invasion of the host. FEMS Microbiol Rev. 2012;36(6):1122-1180. doi:10.1111/j.1574-6976.2012.00340.x

405. Rowe PC, Fontaine KR, Lauver M, et al. Neuromuscular Strain Increases Symptom Intensity in Chronic Fatigue Syndrome. PLOS ONE. 2016;11(7). doi:10.1371/journal.pone.0159386 
406. Wolf JA, Stys PK, Lusardi T, Meaney D, Smith DH. Traumatic axonal injury induces calcium influx modulated by tetrodotoxin-sensitive sodium channels. J Neurosci Off J Soc Neurosci. 2001;21(6):1923-1930.

407. Arundine $M$, Aarts $M$, Lau $A$, Tymianski $M$. Vulnerability of central neurons to secondary insults after in vitro mechanical stretch. J Neurosci Off J Soc Neurosci. 2004;24(37):8106-8123. doi:10.1523/JNEUROSCI.1362-04.2004

408. Liu XZ, Xu XM, Hu R, et al. Neuronal and glial apoptosis after traumatic spinal cord injury. J Neurosci Off J Soc Neurosci. 1997;17(14):5395-5406.

409. Koumlis S, Cheng H, Morgan TE, Finch CE, Eliasson V. Glial Model for Traumatic Brain Injury: Network Strain Field and Inflammation Induced by Repeated Mechanical Impacts In Vitro. Exp Mech. 2018;58(1):125-135. doi:10.1007/s11340-017-0338-3

410. Nordin LE, Möller MC, Julin P, Bartfai A, Hashim F, Li T-Q. Post mTBI fatigue is associated with abnormal brain functional connectivity. Sci Rep. 2016;6(1):21183. doi:10.1038/srep21183

411. Clark DPQ, Perreau VM, Shultz SR, et al. Inflammation in Traumatic Brain Injury: Roles for Toxic A1 Astrocytes and Microglial-Astrocytic Crosstalk. Neurochem Res. 2019;44(6):14101424. doi:10.1007/s11064-019-02721-8

412. Gwak YS, Hulsebosch CE. Remote astrocytic and microglial activation modulates neuronal hyperexcitability and below-level neuropathic pain after spinal injury in rat. Neuroscience. 2009;161(3):895-903. doi:10.1016/j.neuroscience.2009.03.055

413. Jason LA, Porter N, Herrington J, Sorenson M, Kubow S. Kindling and Oxidative Stress as Contributors to Myalgic Encephalomyelitis/Chronic Fatigue Syndrome. J Behav Neurosci Res. 2009; 7(2):1-17.

414. Cooper MS, Clark VP. Neuroinflammation, neuroautoimmunity, and the co-morbidities of complex regional pain syndrome. J Neuroimmune Pharmacol Off J Soc Neurolmmune Pharmacol. 2013;8(3):452-469. doi:10.1007/s11481-012-9392-x

415. Stam CJ. Modern network science of neurological disorders. Nat Rev Neurosci. 2014;15(10):683-695. doi:10.1038/nrn3801

416. Nichols DE, Johnson MW, Nichols CD. Psychedelics as Medicines: An Emerging New Paradigm. Clin Pharmacol Ther. 2017;101(2):209-219. doi:10.1002/cpt.557

417. Komaroff AL, Bateman L. Will COVID-19 Lead to Myalgic Encephalomyelitis/Chronic Fatigue Syndrome? Front Med. 2021;7. doi:10.3389/fmed.2020.606824

418. Lu H, Jaime S, Yang Y. Origins of the Resting-State Functional MRI Signal: Potential Limitations of the "Neurocentric" Model. Front Neurosci. 2019;13:1136. doi:10.3389/fnins.2019.01136

419. Mondelli V, Pariante $\mathrm{CM}$. What can neuroimmunology teach us about the symptoms of longCOVID? Oxf Open Immunol. 2021;2(1). doi:10.1093/oxfimm/iqab004

420. Smith AK, Simon JS, Gustafson EL, et al. Association of a polymorphism in the indoleamine2,3-dioxygenase gene and interferon- $\alpha$-induced depression in patients with chronic hepatitis C. Mol Psychiatry. 2012;17(8):781-789. doi:10.1038/mp.2011.67 
421. Perez M, Jaundoo R, Hilton K, et al. Genetic Predisposition for Immune System, Hormone, and Metabolic Dysfunction in Myalgic Encephalomyelitis/Chronic Fatigue Syndrome: A Pilot Study. Front Pediatr. 2019;7. doi:10.3389/fped.2019.00206

422. Davis HE, Assaf GS, McCorkell L, et al. Characterizing long COVID in an international cohort: 7 months of symptoms and their impact. EClinicalMedicine. 2021;0(0).

doi:10.1016/j.eclinm.2021.101019

423. Seeßle J, Waterboer T, Hippchen T, et al. Persistent symptoms in adult patients one year after COVID-19: a prospective cohort study. Clin Infect Dis. 2021;(ciab611). doi:10.1093/cid/ciab611

424. Townsend L, Moloney D, Finucane C, et al. Fatigue following COVID-19 infection is not associated with autonomic dysfunction. PLOS ONE. 2021;16(2):e0247280. doi:10.1371/journal.pone.0247280

425. Prevalence of ongoing symptoms following coronavirus (COVID-19) infection in the UK - Office for National Statistics. Accessed June 16, 2021.

https://www.ons.gov.uk/peoplepopulationandcommunity/healthandsocialcare/conditionsand diseases/bulletins/prevalenceofongoingsymptomsfollowingcoronaviruscovid19infectioninthe uk/4june2021

426. Group P-CC, Evans RA, McAuley H, et al. Physical, cognitive and mental health impacts of COVID-19 following hospitalisation - a multi-centre prospective cohort study. medRxiv. Published online March 25, 2021:2021.03.22.21254057. doi:10.1101/2021.03.22.21254057

427. Gaebler C, Wang Z, Lorenzi JCC, et al. Evolution of antibody immunity to SARS-CoV-2. Nature. 2021;591(7851):639-644. doi:10.1038/s41586-021-03207-w

428. Cheung CCL, Goh D, Lim X, et al. Residual SARS-CoV-2 viral antigens detected in GI and hepatic tissues from five recovered patients with COVID-19. Gut. Published online June 2, 2021:gutjnl2021-324280. doi:10.1136/gutjnl-2021-324280

429. Böszörményi KP, Stammes MA, Fagrouch ZC, et al. The Post-Acute Phase of SARS-CoV-2 Infection in Two Macaque Species Is Associated with Signs of Ongoing Virus Replication and Pathology in Pulmonary and Extrapulmonary Tissues. Viruses. 2021;13(8):1673. doi:10.3390/v13081673

430. Crunfli F, Carregari VC, Veras FP, et al. SARS-CoV-2 infects brain astrocytes of COVID-19 patients and impairs neuronal viability. medRxiv. Published online February 7 , 2021:2020.10.09.20207464. doi:10.1101/2020.10.09.20207464

431. Andrews MG, Mukhtar T, Eze UC, et al. Tropism of SARS-CoV-2 for Developing Human Cortical Astrocytes. bioRxiv. Published online January 18, 2021:2021.01.17.427024. doi:10.1101/2021.01.17.427024

432. Bocci M, Oudenaarden $C$, Sàenz-Sardà $X$, et al. Infection of brain pericytes underlying neuropathology of COVID-19 patients. bioRxiv. Published online May 25, 2021:2021.05.24.445532. doi:10.1101/2021.05.24.445532

433. Kantonen J, Mahzabin S, Mäyränpää MI, et al. Neuropathologic features of four autopsied COVID-19 patients. Brain Pathol Zurich Switz. 2020;30(6):1012-1016. doi:10.1111/bpa.12889 
434. Visvabharathy L, Hanson BA, Orban Z, et al. Neuro-COVID Long-Haulers Exhibit Broad Dysfunction in T Cell Memory Generation and Responses to Vaccination.; 2021:2021.08.08.21261763. doi:10.1101/2021.08.08.21261763

435. Gold JE, Okyay RA, Licht WE, Hurley DJ. Investigation of Long COVID Prevalence and Its Relationship to Epstein-Barr Virus Reactivation. Pathogens. 2021;10(6):763. doi:10.3390/pathogens10060763

436. Phetsouphanh C, Darley D, Howe A, et al. Immunological dysfunction persists for 8 months following initial mild-moderate SARS-CoV-2 infection. medRxiv. Published online June 3 , 2021:2021.06.01.21257759. doi:10.1101/2021.06.01.21257759

437. Peluso MJ, Lu S, Tang AF, et al. Markers of immune activation and inflammation in individuals with post-acute sequelae of SARS-CoV-2 infection. medRxiv. Published online July 11, 2021:2021.07.09.21260287. doi:10.1101/2021.07.09.21260287

438. Singh I, Joseph P, Heerdt PM, et al. Persistent Exertional Intolerance after COVID-19: Insights from Invasive Cardiopulmonary Exercise Testing. Chest. Published online August 10, 2021:S0012-3692(21)03635-7. doi:10.1016/j.chest.2021.08.010

439. Talla A, Vasaikar SV, Lemos MP, et al. Longitudinal immune dynamics of mild COVID-19 define signatures of recovery and persistence. bioRxiv. Published online June 11, 2021:2021.05.26.442666. doi:10.1101/2021.05.26.442666

440. Sante GD, Buonsenso D, Rose CD, et al. Immune profile of children with post-acute sequelae of SARS-CoV-2 infection (Long Covid). medRxiv. Published online May 10, 2021:2021.05.07.21256539. doi:10.1101/2021.05.07.21256539

441. LongCovidSOS. LongCOVIDSOS Vaccine Report. Accessed June 16, 2021. https://3ca26cd7266e-4609-b25f-

6f3d1497c4cf.filesusr.com/ugd/8bd4fe_a338597f76bf4279a851a7a4cb0e0a74.pdf

442. Tran V-T, Perrodeau E, Saldanha J, Pane I, Ravaud P. Efficacy of COVID-19 Vaccination on the Symptoms of Patients With Long COVID: A Target Trial Emulation Using Data From the ComPaRe e-Cohort in France. Social Science Research Network; 2021. Accessed October 26, 2021. https://papers.ssrn.com/abstract=3932953

443. Wisnivesky JP, Govindarajulu U, Bagiella E, et al. Association of Vaccination With the Persistence of Post-COVID Symptoms. Social Science Research Network; 2021. doi:10.2139/ssrn.3936501

444. Franke C, Ferse C, Kreye J, et al. High frequency of cerebrospinal fluid autoantibodies in COVID-19 patients with neurological symptoms. Brain Behav Immun. 2021;93:415-419. doi:10.1016/j.bbi.2020.12.022

445. Dreyer N, Petruski-Ivleva N, Albert L, et al. Identification of a Vulnerable Group for Post-Acute Sequelae of SARS-CoV-2 (PASC): People with Autoimmune Diseases Recover More Slowly from COVID-19. Int J Gen Med. 2021;14:3941-3949. doi:10.2147/IJGM.S313486

446. Khamsi R. Rogue antibodies could be driving severe COVID-19. Nature. 2021;590(7844):29-31. doi:10.1038/d41586-021-00149-1 
447. Johnson \& Johnson Statement on COVID-19 Vaccine (7/12) | Johnson \& Johnson. Content Lab U.S. Accessed July 16, 2021. https://www.jnj.com/johnson-johnson-july-12-statement-oncovid-19-vaccine

448. Further patients benefit from drug against Long COVID > Friedrich-Alexander-Universität Erlangen-Nürnberg. Accessed August 31, 2021.

https://www.fau.eu/2021/08/27/news/research/further-patients-benefit-from-drug-againstlong-covid/

449. Zhou Y, Xu J, Hou Y, et al. Network medicine links SARS-CoV-2/COVID-19 infection to brain microvascular injury and neuroinflammation in dementia-like cognitive impairment.

Alzheimers Res Ther. 2021;13(1):110. doi:10.1186/s13195-021-00850-3

450. Natalello G, Luca GD, Gigante L, et al. Nailfold capillaroscopy findings in patients with coronavirus disease 2019: Broadening the spectrum of COVID-19 microvascular involvement. Microvasc Res. 2021;133:104071. doi:10.1016/j.mvr.2020.104071

451. Nath A. Neurologic complications of coronavirus infections. Neurology. 2020;94(19):809-810. doi:10.1212/WNL.0000000000009455

452. Alexander GM. Plasma Exchange Therapy in Patients withComplex Regional Pain Syndrome. Pain Physician. 2015;18;4(4;18):383-394. doi:10.36076/ppj.2015/18/383

453. Vijay K. Toll-like receptors in immunity and inflammatory diseases: Past, present, and future. Int Immunopharmacol. 2018;59:391-412. doi:10.1016/j.intimp.2018.03.002

454. Fogarty $\mathrm{H}$, Townsend L, Morrin $\mathrm{H}$, et al. Persistent Endotheliopathy in the Pathogenesis of Long COVID Syndrome. J Thromb Haemost. n/a(n/a). doi:10.1111/jth.15490

455. Pretorius $\mathrm{E}$, Vlok $\mathrm{M}$, Venter $\mathrm{C}$, et al. Persistent clotting protein pathology in Long COVID/PostAcute Sequelae of COVID-19 (PASC) is accompanied by increased levels of antiplasmin. Cardiovasc Diabetol. 2021;20(1):172. doi:10.1186/s12933-021-01359-7

456. Patterson BK, Francisco EB, Yogendra R, et al. Persistence of SARS CoV-2 S1 Protein in CD16+ Monocytes in Post-Acute Sequelae of COVID-19 (PASC) Up to 15 Months Post-Infection. bioRxiv. Published online July 9, 2021:2021.06.25.449905. doi:10.1101/2021.06.25.449905

457. Johnson C. Has Bruce Patterson Cracked Long COVID? Health Rising. Published July 21, 2021. Accessed July 24, 2021. https://www.healthrising.org/blog/2021/07/21/patterson-crackedlong-covid/

458. Martin-Blondel G, Brassat D, Bauer J, Lassmann H, Liblau RS. CCR5 blockade for neuroinflammatory diseases - beyond control of HIV. Nat Rev Neurol. 2016;12(2):95-105. doi:10.1038/nrneurol.2015.248

459. Louboutin J-P, Strayer DS. Relationship between the chemokine receptor CCR5 and microglia in neurological disorders: consequences of targeting CCR5 on neuroinflammation, neuronal death and regeneration in a model of epilepsy. CNS Neurol Disord Drug Targets. 2013;12(6):815-829. doi:10.2174/18715273113126660173

460. Low-Dose Maraviroc, an Antiretroviral Drug, Attenuates the Infiltration of T Cells into the Central Nervous System and Protects the Nigrostriatum in Hemiparkinsonian Monkeys | The Journal of Immunology. Accessed July 24, 2021. https://www.jimmunol.org/content/202/12/3412 
461. Friedman-Levi Y, Liraz-Zaltsman S, Shemesh C, et al. Pharmacological blockers of CCR5 and CXCR4 improve recovery after traumatic brain injury. Exp Neurol. 2021;338:113604. doi:10.1016/j.expneurol.2021.113604

462. Dani M, Dirksen A, Taraborrelli P, et al. Autonomic dysfunction in "long COVID": rationale, physiology and management strategies. Clin Med Lond Engl. 2021;21(1):e63-e67. doi:10.7861/clinmed.2020-0896

463. Adler TE, Norcliffe-Kaufmann L, Condos R, et al. HEART RATE VARIABILITY IS REDUCED 3- AND 6-MONTHS AFTER HOSPITALIZATION FOR COVID-19 INFECTION. J Am Coll Cardiol. 2021;77(18, Supplement 1):3062. doi:10.1016/S0735-1097(21)04417-X

464. Morand A, Campion J-Y, Lepine A, et al. Similar patterns of [18F]-FDG brain PET hypometabolism in paediatric and adult patients with long COVID: a paediatric case series. Eur J Nucl Med Mol Imaging. Published online August 19, 2021. doi:10.1007/s00259-021-05528-4

465. Guedj E, Campion JY, Dudouet P, et al. 18F-FDG brain PET hypometabolism in patients with long COVID. Eur J Nucl Med Mol Imaging. Published online January 26, 2021:1-11. doi:10.1007/s00259-021-05215-4

466. Schwabenland M, Salié H, Tanevski J, et al. Deep spatial profiling of human COVID-19 brains reveals neuroinflammation with distinct microanatomical microglia-T cell interactions. Immunity. 2021;0(0). doi:10.1016/j.immuni.2021.06.002

467. Philippens IHCHM, Böszörményi KP, Wubben JA, et al. SARS-CoV-2 causes brain inflammation and induces Lewy body formation in macaques. bioRxiv. Published online May 5, 2021:2021.02.23.432474. doi:10.1101/2021.02.23.432474

468. Sun B, Tang N, Peluso MJ, et al. Characterization and Biomarker Analyses of Post-COVID-19 Complications and Neurological Manifestations. Cells. 2021;10(2):386. doi:10.3390/cells10020386

469. Brain imaging before and after COVID-19 in UK Biobank | medRxiv. Accessed October 26, 2021. https://www.medrxiv.org/content/10.1101/2021.06.11.21258690v3

470. Weinstock LB, Brook JB, Walters AS, Goris A, Afrin LB, Molderings GJ. Mast cell activation symptoms are prevalent in Long-COVID. Int J Infect Dis IJID Off Publ Int Soc Infect Dis. 2021;112:217-226. doi:10.1016/j.ijid.2021.09.043

471. Mukherjee R, Bhattacharya A, Bojkova D, et al. Famotidine inhibits Toll-like receptor 3mediated inflammatory signaling in SARS-CoV2 infection. J Biol Chem. Published online June 30, 2021:100925. doi:10.1016/j.jbc.2021.100925

472. Li Y, Fu L, Gonzales DM, Lavi E. Coronavirus neurovirulence correlates with the ability of the virus to induce proinflammatory cytokine signals from astrocytes and microglia. J Virol. 2004;78(7):3398-3406. doi:10.1128/jvi.78.7.3398-3406.2004

473. Tremblay M-E, Madore C, Bordeleau M, Tian L, Verkhratsky A. Neuropathobiology of COVID19: The Role for Glia. Front Cell Neurosci. 2020;14. doi:10.3389/fncel.2020.592214

474. Vargas G, Medeiros Geraldo LH, Gedeão Salomão N, Viana Paes M, Regina Souza Lima F, Carvalho Alcantara Gomes F. Severe acute respiratory syndrome coronavirus 2 (SARS-CoV-2) and glial cells: Insights and perspectives. Brain Behav Immun - Health. 2020;7:100127. doi:10.1016/j.bbih.2020.100127 
475. Matschke J, Lütgehetmann M, Hagel C, et al. Neuropathology of patients with COVID-19 in Germany: a post-mortem case series. Lancet Neurol. 2020;19(11):919-929. doi:10.1016/S1474-4422(20)30308-2

476. Kanberg N, Ashton NJ, Andersson L-M, et al. Neurochemical evidence of astrocytic and neuronal injury commonly found in COVID-19. Neurology. 2020;95(12):e1754-e1759. doi:10.1212/WNL.0000000000010111

477. Solomon T. Neurological infection with SARS-CoV-2 - the story so far. Nat Rev Neurol. 2021;17(2):65-66. doi:10.1038/s41582-020-00453-w

478. Kanberg N, Simrén J, Edén A, et al. Neurochemical signs of astrocytic and neuronal injury in acute COVID-19 normalizes during long-term follow-up. EBioMedicine. 2021;70. doi:10.1016/j.ebiom.2021.103512

479. Weiss JM, Downie SA, Lyman WD, Berman JW. Astrocyte-derived monocyte-chemoattractant protein-1 directs the transmigration of leukocytes across a model of the human blood-brain barrier. J Immunol Baltim Md 1950. 1998;161(12):6896-6903.

480. He M, Dong $\mathrm{H}$, Huang $\mathrm{Y}$, et al. Astrocyte-Derived CCL2 is Associated with M1 Activation and Recruitment of Cultured Microglial Cells. Cell Physiol Biochem. 2016;38(3):859-870. doi:10.1159/000443040

481. SARS-CoV-2 causes human BBB injury and neuroinflammation indirectly in a linked organ chip platform | bioRxiv. Accessed October 26, 2021.

https://www.biorxiv.org/content/10.1101/2021.10.05.463205v1

482. Yang AC, Kern F, Losada PM, et al. Dysregulation of brain and choroid plexus cell types in severe COVID-19. Nature. Published online June 21, 2021:1-7. doi:10.1038/s41586-02103710-0

483. Sollini M, Morbelli S, Ciccarelli M, et al. Long COVID hallmarks on [18F]FDG-PET/CT: a casecontrol study. Eur J Nucl Med Mol Imaging. Published online March 7, 2021:1-11. doi:10.1007/s00259-021-05294-3

484. Yong SJ. Persistent Brainstem Dysfunction in Long-COVID: A Hypothesis. ACS Chem Neurosci. 2021;12(4):573-580. doi:10.1021/acschemneuro.0c00793

485. Cheray M, Joseph B. Epigenetics Control Microglia Plasticity. Front Cell Neurosci. 2018;12. doi:10.3389/fncel.2018.00243 